\title{
NONCOMMUTATIVE SYMMETRIC FUNCTIONS II: TRANSFORMATIONS OF ALPHABETS
}

D. Krob ${ }^{1}$, B. Leclerc ${ }^{2}$ and J.-Y. Thibon ${ }^{3}$

${ }^{1}$ L.I.T.P., Université Paris 7, 2 Place Jussieu, 75251 Paris Cedex 05, France

${ }^{2}$ Université de Caen, Département de Mathématiques, Esplanade de la Paix, BP 5186, 14032 Caen cedex, France

${ }^{3}$ Institut Gaspard Monge, Université de Marne-la-Vallée, 2 rue de la Butte-Verte, 93166 Noisy-le-Grand Cedex, France 


\begin{abstract}
Noncommutative analogues of classical operations on symmetric functions are investigated, and applied to the description of idempotents and nilpotents in descent algebras. Its is shown that any sequence of Lie idempotents (one in each descent algebra) gives rise to a complete set of indecomposable orthogonal idempotents of each descent algebra, and various deformations of the classical sequences of Lie idempotents are obtained. In particular, we obtain several $q$-analogues of the Eulerian idempotents and of the GarsiaReutenauer idempotents.
\end{abstract}

\title{
Résumé
}

Nous étudions des analogues non-commutatifs de tranformations classiques sur les fonctions symétriques, et nous les appliquons à la description des idempotents et des nilpotents dans les algèbres de descentes. On montre en particulier que toute suite d'idempotents de Lie (un pour chaque algèbre de descentes) permet de construire une famille complète d'idempotents orthogonaux indécomposables, et diverses déformations des suites classiques d'idempotents de Lie sont obtenues. En particulier, nous obtenons plusieurs $q$-analogues des idempotents eulériens et des idempotents de Garsia-Reutenauer. 
1 Introduction $\quad 3$

2 Background $\quad 5$

2.1 The algebra of noncommutative symmetric functions . . . . . . . . . . . . 5

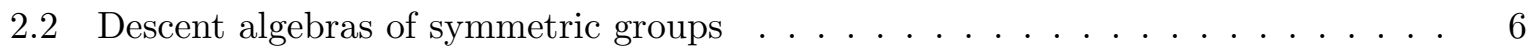

2.3 The star involution . . . . . . . . . . . . . . . . . . 6

3 Idempotents and nilpotents in descent algebras $\quad 8$

3.1 Lie idempotents . . . . . . . . . . . . . . . . . . . 8

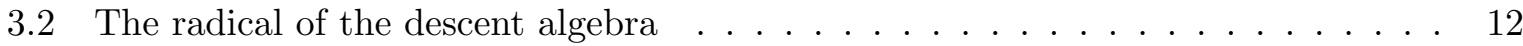

3.3 The Garsia-Reutenauer idempotents . . . . . . . . . . . . . . . . . 13

3.4 Orthogonal idempotents associated with Lie idempotents . . . . . . . . . . . . 15

3.5 Characters of the associated $G L(N)$-modules $\ldots \ldots \ldots \ldots \ldots$

3.6 Dimensions of the quasi-ideals $e_{\lambda} \Sigma_{n} e_{\mu} \ldots \ldots \ldots \ldots \ldots \ldots$

4 Sums, differences and products of alphabets $\quad 21$

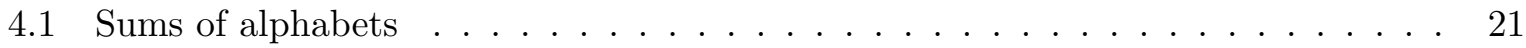

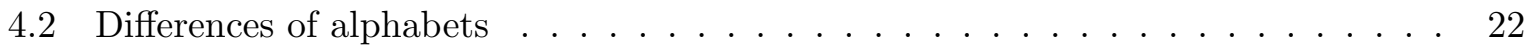

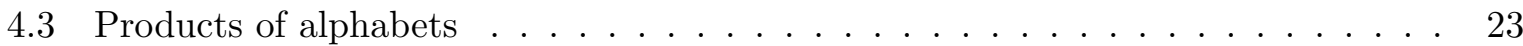

5 Symmetric functions of the alphabet $(1-q) A \quad \mathbf{2 9}$

5.1 Complete functions . . . . . . . . . . . . . . . . . . . . . . 29

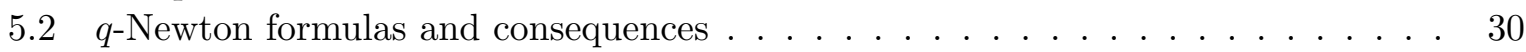

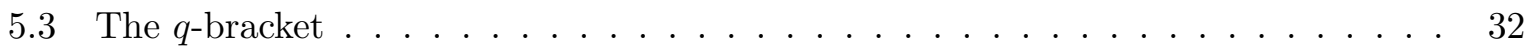

5.4 Diagonalization of the left $q$-bracketing $\ldots \ldots \ldots \ldots$

5.5 Specializations of the family $\pi_{n}(q) \ldots \ldots \ldots \ldots \ldots \ldots$

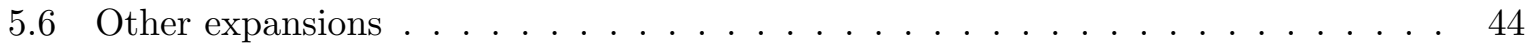

$5.6 .1 \quad S_{n}((1-q) A)$ and $\Lambda_{n}((1-q) A) \ldots \ldots \ldots \ldots 4$

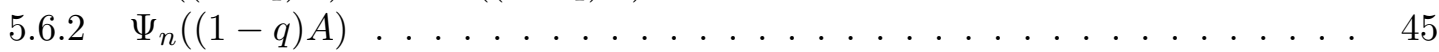

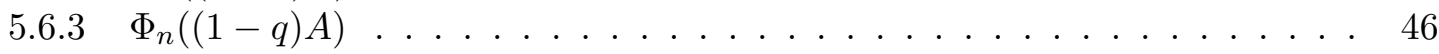

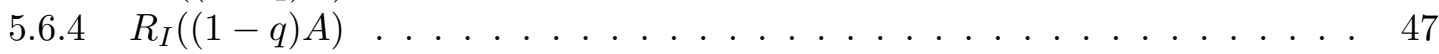

6 Symmetric functions of the alphabet $A /(1-q) \quad 50$

6.1 Complete functions . . . . . . . . . . . . . . . . . . . . 50

6.2 Klyachko's idempotent . . . . . . . . . . . . . . . . . 52

6.3 Other symmetric functions . . . . . . . . . . . . . . . . . 53

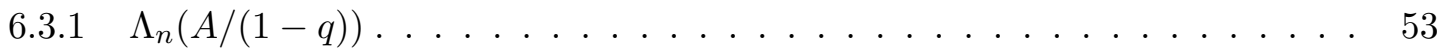

$6.3 .2 \Psi_{n}(A /(1-q)) \ldots \ldots \ldots \ldots \ldots \ldots \ldots$

6.4 A one-parameter family of Lie idempotents . . . . . . . . . 57

7 Symmetric functions of the alphabet $\frac{\mid 1-t}{1-q \mid} A \quad \mathbf{6 0}$

7.1 Complete functions . . . . . . . . . . . . . . . . 60

7.2 Eulerian idempotents associated to Dynkin's projectors . . . . . . . . . . 66

7.3 A $t$-analog of Klyachko's idempotent . . . . . . . . . . . . . . . . . 67

8 Symmetric functions of the alphabet $\frac{1-t \mid}{\mid 1-q} A \quad 69$

8.1 Complete functions . . . . . . . . . . . . . . . . . . . 69

8.2 Symmetric functions of the alphabet $\frac{1-q^{n} \mid}{\mid 1-q} A \ldots \ldots \ldots \ldots$

8.2.1 Klyachko's idempotent again . . . . . . . . . . . . 70 
9 Lie quasi-idempotents as Lie polynomials $\quad \mathbf{7 3}$

9.1 Left derivative . . . . . . . . . . . . . . . . . . 73

9.2 Multilinear Lie polynomials . . . . . . . . . . . . . . . . . . . . 74

9.3 Decompositions on other Lie bases . . . . . . . . . . . . . . . . . 76 


\section{Introduction}

The algebra of noncommutative symmetric functions, introduced in [12], is the free associative algebra over an infinite sequence $\left(\Lambda_{n}\right)_{n \geq 1}$ of noncommuting indeterminates (corresponding to the elementary symmetric functions), endowed with some extra structure imitated from the algebra of commutative symmetric functions. The coefficients are taken in some field $K$ of characteristic zero.

Most of the numerous determinantal formulas of the commutative theory can be adapted to the noncommutative case by means of the notion of quasi-determinant, defined in [13] and further developed in [14, 18]. This point of view leads to a simple and unified presentation of several topics, such as noncommutative continued fractions, Padé approximants, orthogonal polynomials, and various generalizations of the determinant and characteristic polynomial of noncommutative matrices arising in the study of enveloping algebras and their quantum analogs $[12,26]$.

The characteristic feature of these quasi-determinantal computations is that they take place in the skew field generated over $K$ by the $\Lambda_{n}$, rather than in the free algebra Sym $=K\left\langle\Lambda_{1}, \Lambda_{2}, \ldots\right\rangle$ of integral noncommutative symmetric functions. At the time of writing, the main applications of this algebra come from the following realization. Introducing on Sym the structure of a graded algebra by means of the weight function $w\left(\Lambda_{n}\right)=n$, one can identify it with the direct sum $\bigoplus_{n \geq 0} \Sigma_{n}$ of the descent algebras of all symmetric groups, endowed with a convolution product coming from the interpretation of permutations as endomorphisms of tensor spaces (see e.g. [33]).

It has been observed for some time that those elements of the algebra of the symmetric group that are relevant to the study of free Lie algebras generally belong to the descent algebra. This is true, for example, of the idempotents of Dynkin-Specht-Wever [9, 36, 38], Solomon [35] and Klyachko [17] (projecting onto Lie elements), of the Eulerian idempotents $[25,32,20,28]$, and of the projectors associated to the canonical decomposition of the free algebra interpreted as the symmetric algebra of a free Lie algebra (see [33]). The interpretation of these distinguished elements as noncommutative symmetric funtions leads to a simple explanation of their properties, and to a systematic method of calculation, which is a perfect noncommutative analog of the use of symmetric functions for calculating with characters of symmetric groups.

In this paper, we pursue the study of integral noncommutative symmetric functions and of their applications to the combinatorics of free Lie algebras, from the following point of view. As it appears that the descent algebra has to be considered as a noncommutative analog of the character ring, we systematically try to extend to the noncommutative case the operations on commutative symmetric functions encountered in character computations.

Among them are the transformations $A \rightarrow(1-q) A, A \rightarrow A /(1-q)$ and $A \rightarrow \frac{1-t}{1-q} A$ of the argument of the symmetric functions (called here the alphabet), which means that the power-sums $\psi_{k}(A)=\sum a_{i}^{k}$ are replaced by $\left(1-q^{k}\right) \psi_{k}(A), \psi_{k}(A) /\left(1-q^{k}\right)$ and so on. These transformations, which are of fundamental importance in the theory of Hall-Littlewood and Macdonald functions, arise for example in the computation of character tables of linear groups over finite fields (see [21]) and of their Hecke algebras (cf. [31]), and in the description of the representations of the symmetric group in the cohomology rings of flag manifolds. 
Noncommutative analogs of these transformations can be consistently defined, and one obtains from them new interesting elements of the descent algebras. For example, one can exhibit a naturally defined one-parameter family of Lie idempotents interpolating between the classical ones, and more general deformations can be obtained without greater difficulty. It is also shown that any infinite sequence $\left(\pi_{n}\right)_{n \geq 1}$ of Lie idempotents gives rise to decompositions of the descent algebras in the sense of [11], i.e. to a complete set of orthogonal idempotents for each descent algebra. Thus, we obtain $q$-analogs and other deformations of the Garsia-Reutenauer idempotents, and as a consequence, of the Eulerian idempotents. An interesting example of this situation is provided by the spectral projectors of the iterated $q$-bracketing.

This article is organized as follows. We first recall the necessary background on noncommutative symmetric functions (Section 2). Then, we study idempotents and nilpotents in descent algebras from the point of view of noncommutative symmetric functions. The known results are reproved and generalized. In particular, we obtain a complete orthogonal set of minimal idempotents for each descent algebra from any sequence of Lie idempotents. It is shown that these minimal idempotents $e_{\lambda}$ (which are indexed by partitions $\lambda$ ) generate, for a given $\lambda$, isomorphic ideals in the algebra of the symmetric group, and that the dimensions of the quasi-ideals $e_{\lambda} \Sigma_{n} e_{\mu}$ of the descent algebra are also independent of the initial sequence of Lie idempotents (Section 3). Then, we define, imitating the $\lambda$-ring formalism of the commutative theory, sums, differences and products of (virtual) noncommutative alphabets, and we establish the basic properties of these operations (Section 4). Some fundamental examples are developed in the next four Sections. The transformation $A \rightarrow(1-q) A$ (Section 5) is related to the $q$-bracket $[a, b]_{q}=a b-q b a$. For $q=1$, the iterated left bracketing operator $a_{1} a_{2} \cdots a_{n}=\left[\ldots\left[\left[a_{1}, a_{2}\right], a_{3}, \ldots, a_{n}\right]\right.$ is a projector (Dynkin's theorem), but for generic values of $q$, the iterated $q$-bracketing is an invertible operator, with a distinct eigenvalue for each partition of $n$. The spectral projectors of this operator are computed, and it is shown that they are obtained from an interesting family of Lie idempotents by the construction of Section 3. The consideration of the inverse transformation $A \rightarrow A /(1-q)$ leads to interesting formulas, and in particular to a one-parameter family of Lie idempotents, giving all the previously known examples for special values of the parameter. These considerations are then extended to the two natural types of two-parameter deformations (Sections 7 and 8), and explicit formulas are given for some families of symmetric functions. Finally, the study of Lie quasi-idempotents in the descent algebra is completed by their description in some natural bases of the multilinear component of the free Lie algebra (Section 9).

Acknowledgements This work has been supported by the EC network "Algebraic Combinatorics" (Human Capital and Mobility) and the "PRC Mathématiques et Informatique" (CNRS). 


\section{Background}

The material in this section is taken from [12], to which the reader is referred for further details.

\subsection{The algebra of noncommutative symmetric functions}

Let $K$ be a field of characteristic 0 . The algebra of formal noncommutative symmetric functions over $K$ is the free associative algebra $\mathbf{S y m}=K\left\langle\Lambda_{1}, \Lambda_{2}, \ldots,\right\rangle$ generated over $K$ by an infinite sequence of noncommuting indeterminates $\Lambda_{k}$, called the elementary symmetric functions. It is convenient to set $\Lambda_{0}=1$. Let $t$ be another indeterminate, commuting with the $\Lambda_{k}$. One introduces the generating series

$$
\begin{gathered}
\lambda(t)=\sum_{k \geq 0} t^{k} \Lambda_{k}, \\
\sigma(t)=\sum_{k \geq 0} t^{k} S_{k}=\lambda(-t)^{-1}, \\
\Phi(t)=\sum_{k \geq 1} \frac{1}{k} \Phi_{k} t^{k}=\log \sigma(t) .
\end{gathered}
$$

The $S_{k}$ are called complete symmetric functions, and the $\Phi_{k}$ are the power sums of the second kind. The power sums of the first kind, denoted by $\Psi_{k}$ are the coefficients of the formal series

$$
\psi(t)=\sum_{k \geq 1} t^{k-1} \Psi_{k},
$$

defined by the equation

$$
\psi(t)=\lambda(-t) \frac{d}{d t} \sigma(t) .
$$

The algebra $\mathbf{S y m}$ is graded by the weight function $w$ defined by $w\left(\Lambda_{k}\right)=k$, and its homogeneous component of weight $n$ is denoted by $\mathbf{S y m}_{n}$. If $\left(F_{n}\right)$ is a sequence of noncommutative symmetric functions such that $F_{n} \in \mathbf{S y m}_{n}$, we set for any composition $I=\left(i_{1}, \ldots, i_{r}\right)$

$$
F^{I}=F_{i_{1}} F_{i_{2}} \cdots F_{i_{r}} .
$$

Then, it is not difficult to check that $\left(\Lambda^{I}\right),\left(S^{I}\right),\left(\Phi^{I}\right)$ and $\left(\Psi^{I}\right)$ are homogeneous bases of Sym.

The set of all compositions of a given integer $n$ is equipped with the reverse refinement order, denoted $\preceq$. For example, the compositions $J$ of 5 such that $J \preceq(2,1,2)$ are $(2,1,2)$, $(3,2),(2,3)$ and $(5)$. The basis $\left(R_{I}\right)$ of ribbon Schur functions, originally defined in terms of quasi-determinants in [12], can also be defined by either of the two equivalent equations

$$
S^{I}=\sum_{J \preceq I} R_{J}, \quad R_{I}=\sum_{J \preceq I}(-1)^{\ell(I)-\ell(J)} S^{J},
$$

$\ell(I)$ being the length of the composition $I$.

The commutative image of a noncommutative symmetric function is given by the algebra morphism $\Lambda_{n} \mapsto e_{n}$. Then, $S_{n} \mapsto h_{n}, \Psi_{n} \mapsto p_{n}, \Phi_{n} \mapsto p_{n}$, and the image of $R_{I}$ is the ordinary ribbon Schur function, denoted by $r_{I}$. The definition of ribbon Schur function is due to McMahon (see [22], t. 1, p. 200), and are denoted in his book by $h_{I}$. 


\subsection{Descent algebras of symmetric groups}

Let $\sigma \in \mathfrak{S}_{n}$ be a permutation with descent set $A=\left\{d_{1}<\ldots<d_{k}\right\} \subseteq[n-1]$. The descent composition $I=C(\sigma)$ is the composition $I=\left(i_{1}, \ldots, i_{k+1}\right)$ of $n$ defined by $i_{s}=d_{s}-d_{s-1}$, where $d_{0}=0$ and $d_{k+1}=n$. The sum in the group algebra of all permutations with descent composition $I$ is denoted by $D_{I}$. We also set $I=C(A)$, and conversely, the subset of $[n-1]$ associated to a composition $I$ of $n$ will be denoted by $A=E(I)$. The $D_{I}$ with $|I|=n$ form a basis of a subalgebra $\mathbb{Z}\left[\mathfrak{S}_{n}\right]$, called the descent algebra of $\mathfrak{S}_{n}[34]$. We denote by $\Sigma_{n}$ the same algebra, with scalars extended to our ground field $K$, and we define an isomorphism of graded vector spaces

$$
\alpha: \Sigma=\bigoplus_{n \geq 0} \Sigma_{n} \longrightarrow \mathbf{S y m}=\bigoplus_{n \geq 0} \mathbf{S y m}_{n}
$$

by requiring

$$
\alpha\left(D_{I}\right)=R_{I}
$$

for every composition $I$.

The direct sum $\Sigma$ can be turned into an algebra by extending the natural product of its components $\Sigma_{n}$ by setting $x y=0$ for $x \in \Sigma_{p}$ and $y \in \Sigma_{q}$ with $p \neq q$. Then we define the internal product, denoted $*$, on $\mathbf{S y m}$ by requiring that $\alpha$ be an anti-isomorphism. In other words, we set

$$
F * G=\alpha\left(\alpha^{-1}(G) \alpha^{-1}(F)\right) .
$$

One also defines on Sym a coproduct $\Delta$ by any of the following equivalent conditions:

$$
\Delta S_{n}=\sum_{k=0}^{n} S_{k} \otimes S_{n-k}, \quad \Delta \Lambda_{n}=\sum_{k=0}^{n} \Lambda_{k} \otimes \Lambda_{n-k}
$$

or

$$
\Delta \Phi_{n}=\Phi_{n} \otimes 1+1 \otimes \Phi_{n}, \quad \Delta \Psi_{n}=\Psi_{n} \otimes 1+1 \otimes \Psi_{n},
$$

and the fundamental property for computing with the internal product is the following formula:

Proposition $2.1[12]$ Let $F_{1}, F_{2}, \ldots, F_{r}, G \in \mathbf{S y m}$. Then,

$$
\left(F_{1} F_{2} \cdots F_{r}\right) * G=\mu_{r}\left[\left(F_{1} \otimes \cdots \otimes F_{r}\right) * \Delta^{r} G\right]
$$

where in the right-hand side, $\mu_{r}$ denotes the $r$-fold ordinary multiplication and $*$ stands for the operation induced on $\mathbf{S} \mathbf{y} \mathbf{m}^{\otimes n}$ by $*$.

A detailed study of the descent algebra can be found in [11]. Other relevant references are $[1,3,5,24,27,29]$.

\subsection{The star involution}

We also define an anti-involution $*$ of $\mathbf{S y m}$ by

$$
\left(S_{n}\right)^{*}=S_{n},
$$

and we record for later use the images of the usual bases of Sym under $*$. Define $\check{\Psi}_{n}$ by the generating series

$$
\check{\psi}(t)=\sigma^{\prime}(t) \lambda(-t) .
$$


Proposition 2.2 For any composition I,

$$
\left(S^{I}\right)^{*}=S^{\bar{I}}, \quad\left(\Lambda^{I}\right)^{*}=\Lambda^{\bar{I}}, \quad\left(R_{I}\right)^{*}=R_{\bar{I}}, \quad\left(\Phi^{I}\right)^{*}=\Phi^{\bar{I}}, \quad\left(\Psi^{I}\right)^{*}=\check{\Psi}^{\bar{I}},
$$

where $\bar{I}$ denotes the mirror image of $I$, i.e. the composition obtained by reading I from right to left.

Proof - The relations giving the images by $*$ of $\Lambda^{I}, R_{I}$ and $\Phi^{I}$ are immediate consequences of their expressions in the basis $S^{I}$ (see [12]). To obtain the last formula, we apply $*$ to the equation

$$
\frac{d}{d t} \sigma(A ; t)=\sigma(A ; t) \psi(A ; t)
$$

which yields $\left(\Psi_{n}\right)^{*}=\check{\Psi}_{n}$. The conclusion follows.

Note 2.3 The fact that $\left(R_{I}\right)^{*}=R_{\bar{I}}$ shows that $*$ corresponds to the automorphism of the descent algebra defined by

$$
D \in \Sigma_{n} \longrightarrow \omega_{n} D \omega_{n}
$$

where $\omega_{n}=(n n-1 \ldots 1)$ is the maximal permutation of $\mathfrak{S}_{n}$. 


\section{Idempotents and nilpotents in descent algebras}

\subsection{Lie idempotents}

An element $\pi$ of the group algebra $K\left[\mathfrak{s}_{n}\right]$ is said to be a Lie element if it can be expressed as a Lie polynomial over the alphabet $\{1,2, \ldots, n\}$, permutations being identified with standard words over this alphabet. A Lie (quasi-) idempotent is a Lie element which is (quasi-) idempotent (an element $x$ of an algebra is said to be quasi-idempotent if $x^{2}=c x$ for some scalar $c$ ). For a Lie idempotent, we also require that it generates the same left ideal as the Dynkin element $[\ldots[1,2], 3], \ldots, n]$.

The following result, proved in [12], characterizes the Lie quasi-idempotents in the descent algebra.

Theorem 3.1 Let $F=\alpha(\pi)$ be an element of $\mathbf{S y m}_{n}$, where $\pi \in \Sigma_{n}$.

(i) The following assertions are equivalent:

1. $\pi$ is a Lie quasi-idempotent;

2. $F$ is a primitive element for $\Delta$;

3. $F$ belongs to the Lie algebra $L(\Psi)$.

(ii) Moreover, $\pi$ is a Lie idempotent iff $F-\frac{1}{n} \Psi_{n}$ is in the Lie ideal $[L(\Psi), L(\Psi)]$.

A characterization of Lie idempotents equivalent to (ii) was also obtained by Blessenohl and Laue in [5] (using other methods). As pointed out by C. Reutenauer, Theorem 3.1 is also an easy consequence of Theorem 9.19 of [33].

For the sake of brevity, a noncommutative symmetric function will be said to be a Lie (quasi-) idempotent if it is the image under $\alpha$ of such an element of the descent algebra.

As a first application of the characterization theorem, let us give the following formula.

Proposition 3.2 Let $F_{n}$ be a Lie quasi-idempotent of $\mathbf{S y m}_{n}$ and let I be a composition of $n$. Then,

$$
R_{I} * F_{n}=(-1)^{\ell(I)-1} F_{n}
$$

Proof - Observe first that

$$
S^{I} * F_{n}=\left\{\begin{array}{cl}
F_{n} & \text { if } I=(n) \\
0 & \text { if } I \neq(n)
\end{array} .\right.
$$

Indeed, if $I=\left(i_{1}, \ldots, i_{r}\right)$ with $r>1$, one can write

$$
S^{I} * F_{n}=\mu_{r}\left[S_{i_{1}} \otimes \ldots \otimes S_{i_{r}} * \sum 1 \otimes \ldots \otimes F_{n} \otimes \ldots \otimes 1\right]=0
$$

according to Theorem 3.1. The result follows now from (10) since

$$
R_{I} * F_{n}=\left(\sum_{J \preceq I}(-1)^{\ell(I)-\ell(J)} S^{J}\right) * F_{n}=(-1)^{\ell(I)-1} S_{n} * F_{n}=(-1)^{\ell(I)-1} F_{n} .
$$


Note 3.3 One can also prove Proposition 3.2 by induction on $\ell(I)$, using the fact that

$$
R_{\left(i_{1}, \ldots, i_{r}\right)} * F_{n}=\left(R_{i_{1}} R_{\left(i_{2}, \ldots, i_{r}\right)}-R_{\left(i_{1}+i_{2}, i_{3}, \ldots, i_{r}\right)}\right) * F_{n}=-R_{\left(i_{1}+i_{2}, i_{3}, \ldots, i_{r}\right)} * F_{n} .
$$

Example 3.4 Let $x_{1}, x_{2}, \ldots$ be independent commutative indeterminates and consider the noncommutative symmetric function

$$
\mathbf{K}_{n}\left(x_{1}, \ldots, x_{n-1}\right)=\sum_{|I|=n}\left(\prod_{d \in E(I)} x_{d}\right) R_{I} .
$$

This is a generating function for permutations by descent class, which as we shall see is especially interesting for $x_{i}=q^{i}$. Now let $F_{n}$ be a primitive element of $\mathbf{S y m}_{n}$. Then,

$$
\mathbf{K}_{n} * F_{n}=\prod_{i=1}^{n-1}\left(1-x_{i}\right) \cdot F_{n}
$$

Indeed, using Proposition 3.2, we obtain

$$
\begin{gathered}
\mathbf{K}_{n} * F_{n}=\sum_{|I|=n} \prod_{d \in E(I)}\left(-x_{d}\right) \cdot F_{n} \\
=\sum_{A \subseteq[n-1]} \prod_{d \in A}\left(-x_{d}\right) \cdot F_{n}=\left(1-x_{1}\right)\left(1-x_{2}\right) \cdots\left(1-x_{n-1}\right) \cdot F_{n} .
\end{gathered}
$$

Proposition 3.2 implies the following simple characterization of Lie quasi-idempotents:

Corollary 3.5 Let $F_{n}$ be in $\mathbf{S y m}_{n}$. Then $F_{n}$ is a Lie quasi-idempotent iff one has

$$
R_{1^{k}, n-k} * F_{n}=(-1)^{k} F_{n}
$$

for $k \in[0, n-1]$.

Proof - Proposition 3.2 shows that (13) is satisfied when $F_{n}$ is a Lie quasi-idempotent. Conversely suppose that (13) holds. We then have

$$
\Psi_{n} * F_{n}=\left(\sum_{k=0}^{n-1}(-1)^{k} R_{1^{k}, n-k}\right) * F_{n}=n F_{n}
$$

Example 3.6 Let $\Theta_{n}(q)$ be the noncommutative symmetric function defined by

$$
\Theta_{n}(q)=\sum_{k=0}^{n-1}(-q)^{k} R_{1^{k}, n-k} .
$$

It is the image under $\alpha$ of the iterated $q$-bracketing operator

$$
\eta_{n}(q)=\left[\ldots\left[[1,2]_{q}, 3\right]_{q}, \ldots, n\right]_{q}
$$


(see section 5.3). Formula (13) shows that for a primitive element $F_{n}$,

$$
\Theta_{n}(q) * F_{n}=\left(1+q+q^{2}+\cdots+q^{n-1}\right) F_{n} .
$$

This is a $q$-analogue of Dynkin's characterization of Lie polynomials. Indeed, it means that homogeneous Lie polynomials of degree $n$ are eigenvectors of the iterated $q$-bracketing, with the $q$-integer $[n]_{q}$ as eigenvalue, and moreover, a homogeneous element $P \in K\langle A\rangle_{n}$ is a Lie polynomial iff $P \cdot \eta_{n}(q)=[n]_{q} P$. Another proof of this property in given in the sequel (see Proposition 5.6). For $q=1, n$ is the only nonzero eigenvalue. For generic $q$, there are $p(n)$ (number of partitions of $n$ ) distinct and nonzero eigenvalues, and the corresponding eigenspaces are determined in section 5.4 .

Let us now give the following lemma of independent interest.

Lemma 3.7 Let $F=\left(F_{n}\right)_{n \geq 1}$ be a sequence of primitive elements such that $F_{n}$ belongs to $\mathbf{S y m}_{n}$ for every $n \geq 1$. Then,

$$
\Psi_{n} * F^{I}=i_{1}\left[\left[\ldots\left[F_{i_{1}}, F_{i_{2}}\right], \ldots,\right], F_{i_{r}}\right]
$$

for any composition $I=\left(i_{1}, i_{2}, \ldots, i_{r}\right)$ of $n$.

Proof - Identifying compositions with words over $\mathbb{N}^{*}$ and denoting by $\langle I, J \sqcup K\rangle$ the coefficient of $I$ in the shuffle of the two words $J$ and $K$, one has

$$
\begin{aligned}
\psi(t) * F^{I}=\mu\left[\lambda(-t) \otimes \frac{d}{d t} \sigma(t) * \sum_{P, Q}\langle I, P \sqcup Q\rangle F^{P} \otimes F^{Q}\right] \\
=\left(\sum_{P, Q}\langle I, P \sqcup Q\rangle(-1)^{|P|}|Q| \omega\left(F^{P}\right) F^{Q}\right) t^{|I|-1},
\end{aligned}
$$

since $\Lambda_{n} * F=\omega(F)$ for every $F \in \mathbf{S y m}_{n}$. It follows that

$$
\Psi_{n} * F^{I}=\sum_{P, Q}\langle I, P \sqcup Q\rangle(-1)^{\ell(P)}|Q| F^{\bar{P}} F^{Q},
$$

since one sees from Theorem 3.1 that $\omega\left(F_{n}\right)=(-1)^{n-1} F_{n}$ for every primitive element $F_{n}$. Setting $J=\left(i_{1}, \ldots, i_{r-1}\right)$, we get

$$
\begin{gathered}
\psi(t) * F^{I}=\psi(t) * F^{J} F_{i_{r}} \\
=\mu\left[\lambda(-t) \otimes \frac{d}{d t} \sigma(t) * \sum_{P, Q}\langle J, P \sqcup Q\rangle F^{P} F_{i_{r}} \otimes F^{Q}+\sum_{P, Q}\langle J, P \sqcup Q\rangle F^{P} \otimes F^{Q} F_{i_{r}}\right] \\
=\left(\sum_{P, Q}\langle J, P \sqcup Q\rangle(-1)^{\ell P+1}|Q| F_{i_{r}} F^{\bar{P}} F^{Q}\right. \\
\left.+\sum_{P, Q}\langle J, P \sqcup Q\rangle(-1)^{\ell(P)}\left(|Q|+i_{r}\right) F^{\bar{P}} F^{Q} F_{i_{r}}\right) t^{|I|-1} .
\end{gathered}
$$

It follows from this last equality and relation (15) that

$$
\Psi_{n} * F^{I}=-\left[F_{i_{r}}, \Psi_{|J|} * F^{J}\right]+i_{r}\left(\sum_{P, Q}\langle J, P \sqcup Q\rangle(-1)^{\ell(P)} F^{\bar{P}} F^{Q}\right) F_{i_{r}}
$$




$$
=\left[\Psi_{|J|} * F^{J}, F_{i_{r}}\right]+i_{r} \mu\left[(\tilde{\omega} \otimes 1) \Delta\left(F^{J}\right)\right] F_{i_{r}} .
$$

Using the antipodal property of $\tilde{\omega}$, we finally obtain

$$
\Psi_{n} * F^{I}=\left[\Psi_{|J|} * F^{J}, F_{i_{r}}\right]
$$

from which the desired property follows by induction.

This formula should be compared with the following property of $\Phi_{n}[12]$

$$
\frac{\Phi_{n}}{n} * F^{I}=\left(F_{i_{1}} F_{i_{2}} \cdots F_{i_{r}}\right) \phi_{r}
$$

where $\phi_{r}=\alpha^{-1}\left(\Phi_{r} / r\right)$ acts on the word $F_{i_{1}} F_{i_{2}} \cdots F_{i_{r}}$ by permutation of the subscripts.

Example 3.8 Lemma 3.7 implies that the restriction of the internal product to the Lie ideal $\mathcal{J}=[L(\Psi), L(\Psi)]$ is zero. Indeed, if $G^{I}=G_{i_{1}} \cdots G_{i_{r}} \in \mathbf{S y m}_{n}$ is any product of elements of positive weight, and if $F_{n} \in \mathbf{S y m}_{n}$ is a primitive element, Proposition 2.1 shows that $G^{I} * \Psi_{n}=0$, and in particular that

$$
\Psi^{I} * \Psi_{n}=0 \text { for } \ell(I)>1 .
$$

But $\mathcal{J}$ is spanned by the elements

$$
\Gamma_{I}=i_{1}\left[\ldots\left[\Psi_{i_{1}}, \Psi_{i_{2}}\right], \ldots, \Psi_{i_{r}}\right]=\Psi_{n} * \Psi^{I},
$$

with $\ell(I)>1$, and for any two of these elements,

$$
\Gamma_{I} * \Gamma_{J}=\left(\Psi_{n} * \Psi^{I}\right) *\left(\Psi_{n} * \Psi_{J}\right)=\Psi_{n} *\left(\Psi^{I} * \Psi^{n}\right) * \Psi^{J}=0 .
$$

Moreover, the Lie ideal $\mathcal{J}$ is a two sided ideal for the internal product. To see this, let

$$
x=F * \Gamma_{I} * G=\left(F * \Psi_{n}\right) *\left(\Psi^{I} * G\right) .
$$

Expanding $F$ on the basis $\Psi^{J}$, we see that $F * \Psi_{n}$ is proportional to $\Psi_{n}$, so we may suppose $x=\Gamma_{I} * G=\Psi_{n} *\left(\Psi^{I} * G\right)$. Computing now $\Psi^{I} * G$ by means of Proposition 2.1, we see that the result cannot contain a term in $\Psi_{n}$, i.e.

$$
\Psi^{I} * G=\sum_{\ell(J)>1} c_{J} \Psi^{J}
$$

and thus $x=\sum_{\ell(J)>1} c_{J} \Gamma_{J} \in \mathcal{J}$.

Example 3.9 The Magnus formula (expressing $\Psi_{n}$ in terms of the $\Phi^{I}$, [23]) can also be derived from Lemma 3.7. Indeed, for a sequence of Lie idempotents $F_{n} \in \mathbf{S y m}_{n}$ with generating series $F(t)=\sum_{n \geq 1} F_{n} t^{n}$, formula (14) can be rewritten

$$
\psi(1) *\left(F\left(t_{1}\right) F\left(t_{2}\right) \cdots F\left(t_{r}\right)\right)=\left\{t_{1} F^{\prime}\left(t_{1}\right), F\left(t_{2}\right) \cdots F\left(t_{r}\right)\right\}
$$

where $\left.\left\{a_{1}, a_{2} \cdots a_{r}\right\}=\left[\ldots\left[a_{1}, a_{2}\right], a_{3}\right], \ldots, a_{r}\right]$. Thus,

$$
\begin{gathered}
t \psi(t)=\sum_{n \geq 1} \Psi_{n} t^{n}=\psi(1) * \sigma(t)=\psi(1) * e^{\Phi(t)} \\
=\sum_{n \geq 0} \frac{1}{n !} \psi(1) * \Phi(t)^{n}=\sum_{n \geq 1} \frac{1}{n !}\left\{t \Phi^{\prime}(t), \Phi(t)^{n-1}\right\} \\
=\left\{t \Phi^{\prime}(t), \frac{e^{\Phi(t)}-1}{\Phi(t)}\right\}
\end{gathered}
$$

which is the required expression. 


\subsection{The radical of the descent algebra}

Let $\underline{F} \in$ Sym denote the commutative image of $F \in \mathbf{S y m}$. Solomon's formula for $S^{I} * S^{J}$ implies that

$$
\underline{F * G}=\underline{F} * \underline{G} .
$$

Thus, the commutative image of any nilpotent element is zero, since there exists no nonzero nilpotent for the commutative internal product. Moreover, it follows from (20) that the set

$$
\mathcal{R}=\{F \in \mathbf{S y m}: \underline{F}=0\},
$$

which is the two-sided ideal of Sym generated by $[L(\Psi), L(\Psi)]$, is also a two-sided ideal for the internal product. Clearly, $\mathcal{R}$ contains all the nilpotent elements. It is known that conversely, the elements of $\mathcal{R}$ are nilpotent (cf [34], [11], [1]), i.e. that $\mathcal{R}$ is the nilpotent radical of Sym (for the internal product). This fact is usually derived from the multiplicative properties of the basis $\left(S^{I}\right)$. It can also be derived from the following lemma, which is a direct consequence of Proposition 2.1 and Lemma 3.7. Denote by $\mathfrak{S}(I)$ the set of all distinct reorderings of a composition $I$.

Lemma 3.10 Let $I=\left(i_{1}, \ldots, i_{r}\right)$ and $J=\left(j_{1}, \ldots, j_{s}\right)$ be two compositions of $n$. Then,

(i) if $\ell(J)<\ell(I)$ then $\Psi^{I} * \Psi^{J}=0$.

(ii) if $\ell(J)>\ell(I)$ then $\Psi^{I} * \Psi^{J} \in \operatorname{Vect}\left\langle\Psi^{K}: K \in \mathfrak{S}(J)\right\rangle \cap \mathcal{R}$. More precisely,

$$
\Psi^{I} * \Psi^{J}=\sum_{\substack{J_{1}, \ldots J_{r} \\\left|J_{k}\right|=i_{k}}}\left\langle J, J_{1} \sqcup \cdots J_{r}\right\rangle \Gamma_{J_{1}} \cdots \Gamma_{J_{r}}
$$

where the $\Gamma_{K}$ are defined by (17).

(iii) if $\ell(J)=\ell(I)$, then $\Psi^{I} * \Psi^{J} \neq 0$ only for $J \in \mathfrak{S}(I)$, in which case $\Psi^{I} * \Psi^{J}=c_{I} \Psi^{I}$, where $c_{I}=\prod_{i} i^{m_{i}} m_{i} !, m_{i}$ being the multiplicity of the part $i$ in $I$.

Hence, if $K$ and $H$ are permutations of $I$, then $\Psi^{I} *\left(\Psi^{K}-\Psi^{H}\right)=0$, and thus $\left(\Psi^{K}-\Psi^{H}\right)^{* 2}=0$. Moreover, if $L$ and $M$ are of the same length $r$ as $I$ but are not permutations of $I$, then $\left(\Psi^{M}-\Psi^{L}\right) *\left(\Psi^{K}-\Psi^{H}\right)=0$.

To rephrase this, let $\mathbf{S y m}{ }^{[k]}$ be the subspace of $\mathbf{S y m}$ generated by the $\Psi^{I}$ with $\ell(I)=k$, and $\mathcal{R}^{[k]}=\mathcal{R} \cap \mathbf{S y m}^{[k]}$. Since $\mathcal{R}^{[k]}$ is clearly spanned by the elements $\Psi^{K}-\Psi^{H}$, where $K$ and $H$ are reorderings of the same partition of length $k$, we see that for any two elements $F$ and $G$ of $\mathcal{R}^{[k]}$, one has $F * G=0$. Thus,

$$
\mathcal{R}^{[k]} * \mathcal{R}^{[k]}=\{0\}
$$

and it follows from the lemma that

$$
\text { for } \quad \begin{array}{ll}
i<j & \mathcal{R}^{[j]} * \mathcal{R}^{[i]}=\{0\} \\
& \mathcal{R}^{[i]} * \mathcal{R}^{[j]} \subseteq \mathcal{R}^{[j]}
\end{array}
$$

Thus, if $F=F_{2}+F_{3}+\cdots+F_{n-1} \in \mathcal{R}_{n}$, where $F_{k} \in \mathcal{R}_{n}^{[k]}$, we see that $\left(F_{2}+\cdots+F_{n-1}\right)^{*(n-1)}=$ 0 , since all the terms arising in the expansion of the product are zero by (22), (23) or $(24)$. 
Corollary $3.11[34,11,1]$ The radical of the descent algebra $\Sigma_{n}$ is $R_{n}=\alpha^{-1}\left(\mathcal{R}_{n}\right)$. Moreover, the nilpotency index of $R_{n}$ is $n-1$, that is, for any $x_{1}, \ldots, x_{n-1} \in R_{n}$, the product $x_{1} \cdots x_{n-1}$ is zero.

It follows that $\Sigma_{n} / R_{n}$ is a commutative algebra of dimension $p(n)$, the number of partitions of $n$. The irreducible representations of $\Sigma_{n}$ are thus one-dimensional, and can be labeled by the partitions $\lambda$ of $n$. These representations have been constructed in [11] by means of a complete set of orthogonal idempotents, which are described in the next section. A different construction, closely related to noncommutative symmetric functions, appears in [1].

¿From the above description, one can find the eigenvalues of the elements of the descent algebra in the (left or right) regular representation.

Proposition 3.12 Let $F \in \mathbf{S y m}_{n}$ considered as an algebra for the internal product $*$ and let $f$ be its commutative image. Let $L_{F}$ and $R_{F}$ be the two endomorphisms of $\mathbf{S y m}_{n}$ defined by $L_{F}(G)=F * G$ and $R_{F}(G)=G * F$. Then,

1) The eigenvalues of $R_{F}$ and of $L_{F}$ are the same.

2) The eigenvalues of $R_{F}$ and $L_{F}$ are exactly the eigenvalues of the commutative endomorphism $g \longrightarrow f * g$ (where * denotes here the commutative internal product of $\left.\mathrm{Sym}_{n}\right)$. They are equal to

$$
t_{\lambda}=<f, p_{\lambda}>
$$

where $\lambda$ is a partition of $n$.

Proof - Let $P(x) \in K[x]$ be the minimum polynomial of $L_{F}$, which is also the minimum polynomial of $F$ over $K$ for the internal product. Let also $Q(x)$ be the minimum polynomial of the commutative image $f=\underline{F}$ of $F$, for the commutative internal product. For a polynomial $F(t)$, denote by $F^{*}$ the function on Sym obtained by evaluting $F$ by means of the internal product. Then, since $Q^{*}(F)=Q^{*}(f)=0$, Corollary 3.11 implies that $Q^{*}(F)$ is nilpotent. Thus, $P$ divides some power $Q^{m}$ of $Q$. Also, the corresponding endomorphism $L_{f}: g \mapsto f * g$ of $S y m_{n}$ is clearly semisimple, having for any $f$ the basis $\left(p_{\lambda}\right)$ as eigenbasis. Moreover, the eigenvalue corresponding to $p_{\lambda}$ is given by $t_{\lambda}=\left\langle f, p_{\lambda}\right\rangle$. Thus, the eigenvalues of $L_{F}$ are the scalar products $\left\langle\underline{F}, p_{\lambda}\right\rangle$.

Example 3.13 With $F=S^{I}$, the eigenvalues only depend on the partition $\mu$ of which $I$ is a reordering, and the eigenvalues are the $\left\langle h_{\mu}, p_{\lambda}\right\rangle$, which are the values of a permutation character of the symmetric group, hence are positive integers. For $F=R_{I}$, the eigenvalues are also given by character values, and thus are also integers (not necessarily positive).

\subsection{The Garsia-Reutenauer idempotents}

The Poincaré-Birkhoff-Witt theorem shows that the homogeneous component of order $n$ of the free associative algebra can be decomposed as

$$
K\left\langle a_{1}, a_{2}, \ldots, a_{N}\right\rangle_{n}=\bigoplus_{\lambda \vdash n} S^{\lambda} L\left(a_{1}, a_{2}, \ldots, a_{N}\right)
$$


where $S^{\lambda} L\left(a_{1}, a_{2}, \ldots, a_{N}\right)$ denotes the submodule of $K\left\langle a_{1}, a_{2}, \ldots, a_{N}\right\rangle_{n}$ generated by the symmetrized products of type $\lambda$ of elements of the free Lie algebra $L\left(a_{1}, a_{2}, \ldots, a_{N}\right)$ (i.e. symmetrized products of Lie polynomials of degrees $\left.\lambda_{1}, \lambda_{2} \ldots\right)$. One can prove that all projectors $\pi_{\lambda}$ on the modules $S^{\lambda} L\left(a_{1}, a_{2}, \ldots, a_{N}\right)$ belong to the descent algebra. More precisely, Garsia and Reutenauer [11] showed that

$$
\alpha\left(\pi_{\lambda}\right)=E_{\lambda}(\Phi)=\frac{1}{|\lambda| !} \sum_{\sigma(I)=\lambda} \frac{\Phi^{I}}{\pi(I)}
$$

for every partition $\lambda$ of $n$. It follows that the family $\left(E_{\lambda}(\Phi)\right)_{\lambda \vdash n}$ form a complete family of orthogonal idempotents for the internal product. We give here a simple argument for obtaining this result. Following [11], we introduce the series

$$
\Phi(x)=\sum_{k \geq 1} x_{k} \frac{\Phi_{k}}{k}
$$

where $x=\left(x_{k}\right)$ is a sequence of commutative indeterminates. It is then easy to see that

$$
\exp (\Phi(x))=\sum_{\lambda} x^{\lambda} E_{\lambda}
$$

Lemma 3.14 One has

$$
\Phi(1) * \exp (\Phi(x))=\Phi(x)
$$

Proof - One can write

$$
\Phi(1) * \exp (\Phi(x))=\log \sigma(1) * \exp (\Phi(x))=\sum_{i \geq 1} \frac{(-1)^{k-1}}{k}(\sigma(1)-1)^{k} * \exp (\Phi(x)) .
$$

Thus we are led to compute

$$
\begin{gathered}
(\sigma(1)-1)^{k} * \exp (\Phi(x))=\mu_{k}\left[(\sigma(1)-1)^{\otimes k} * \Delta^{k}(\exp (\Phi(x)))\right] \\
=\mu_{k}\left[(\sigma(1)-1)^{\otimes k} * \exp (\Phi(x))^{\otimes k}\right]=(\exp (\Phi(x))-1)^{k},
\end{gathered}
$$

since $\exp (\Phi(x))$, being the exponential of a primitive element, is a group-like element. Hence we get

$$
\begin{gathered}
\Phi(1) * \exp (\Phi(x))=\log \sigma(1) * \exp (\Phi(x))=\sum_{i \geq 1} \frac{(-1)^{k-1}}{k}(\exp (\Phi(x))-1)^{k} \\
=\log (\exp (\Phi(x)))=\Phi(x) .
\end{gathered}
$$

Lemma 3.14 shows equivalently that

$$
\Phi_{n} * E_{\lambda}=\left\{\begin{array}{cl}
\Phi_{n} & \text { if } \lambda=n \\
0 & \text { if } \lambda \neq n
\end{array} .\right.
$$


Lemma 3.15 One has

$$
\exp (\Phi(x)) * \exp (\Phi(y))=\exp (\Phi(x y))
$$

where $x y$ denotes the sequence $x y=\left(x_{k} y_{k}\right)_{k \geq 1}$.

Proof - We have

$$
\exp (\Phi(x)) * \exp (\Phi(y))=\sum_{k \geq 0} \frac{1}{k !} \Phi(x)^{k} * \exp (\Phi(y)) .
$$

But, using the fact that $\exp (\Phi(y))$ is group-like, one obtains

$$
\Phi(x)^{k} * \exp (\Phi(y))=\mu\left[\Phi(x)^{\otimes k} * \exp (\Phi(y))^{\otimes k}\right]=\Phi(x y)^{k},
$$

since according to Lemma 3.14 ,

$$
\Phi(x) * \exp (\Phi(y))=\sum_{k, \lambda} x_{k} y^{\lambda} \frac{\Phi_{k}}{k} * E_{\lambda}=\Phi(x y) .
$$

Hence,

$$
\exp (\Phi(x)) * \exp (\Phi(y))=\sum_{k \geq 0} \frac{1}{k !} \Phi(x y)^{k}=\exp (\Phi(x y))
$$

An immediate consequence of this lemma is that

$$
E_{\lambda} * E_{\mu}=\delta_{\lambda, \mu} E_{\lambda}
$$

Hence $\left(E_{\lambda}\right)_{\lambda \vdash n}$ is a complete family of orthogonal idempotents, since the decomposition of $S_{n}$ on the basis $\left(\Phi^{I}\right)$ shows that

$$
S_{n}=\sum_{\lambda \vdash n} E_{\lambda}
$$

\subsection{Orthogonal idempotents associated with Lie idempotents}

In fact, the result of the previous section is a particular case of a more general construction that we shall now explain. Let $F=\left(F_{n}\right)$ be a sequence of Lie quasi-idempotents with non-zero normalization factors, $F_{n}$ belonging to $\mathbf{S y m}_{n}$. We define as usual

$$
F^{I}=F_{i_{1}} F_{i_{2}} \ldots F_{i_{n}}
$$

for any composition $I=\left(i_{1}, i_{2}, \ldots, i_{n}\right)$. The family $\left(F^{I}\right)$ is then a basis of $\mathbf{S y m}$ by an obvious triangularity property. It follows that

$$
S_{n}=\sum_{|I|=n} p_{n, I} F^{I}
$$

for some scalar coefficients $\left(p_{n, I}\right)$. Then we associate to a partition $\lambda$ of $n$ the element

$$
E_{\lambda}(F)=\sum_{I \in \mathfrak{S}(\lambda)} p_{n, I} F^{I}
$$


where $\mathfrak{S}(\lambda)$ is the set of all permutations of $\lambda$. Hence we have by construction

$$
S_{n}=\sum_{\lambda \vdash n} E_{\lambda}(F)
$$

Moreover, the following result shows that the Garsia-Reutenauer construction is in fact generic.

Theorem 3.16 The family $\left(E_{\lambda}(F)\right)_{\lambda \vdash n}$ is a complete family of orthogonal idempotents of $\mathbf{S y m}_{n}$.

Proof - Sym may be considered as the free associative algebra generated by the elements $F_{n}$. Hence we can define an algebra automorphism $s_{x}$ of Sym by

$$
s_{x}\left(F_{n}\right)=x_{n} F_{n}
$$

$x=\left(x_{n}\right)_{n \geq 1}$ being as above a sequence of commutative variables. Note that

$$
s_{x}(\sigma(1))=\sum_{\lambda} x^{\lambda} E_{\lambda}(F):=E(x) .
$$

The theorem will be a consequence of the following two lemmas.

Lemma 3.17 The series $E(x)$ is a group-like element for $\Delta$.

Proof - Indeed,

$$
E(x)=s_{x}(\sigma(1))=s_{x}(\exp (\Phi(1)))=\exp \left(s_{x}(\Phi(1))\right),
$$

and since $\Phi_{n} \in L(F)=L(\Phi)$, the series $s_{x}(\Phi(1))$ is a Lie series. Hence $E(x)$, being the exponential of a Lie element, is a group-like element for $\Delta$.

Lemma 3.18 For any $G \in \mathbf{S y m}$,

$$
s_{x}(G)=G * E(x) .
$$

Proof - This is true for $G=S_{n}$, since $s_{x}(\sigma(1)=E(x)=\sigma(1) * E(x)$. Now, the formula is also true for products of complete functions $S^{I}$, because for a product $G H$,

$$
(G H) * E(x)=\mu[(G \otimes H) *(E(x) \otimes E(x))]=(G * E(x)) *(H * E(x)) .
$$

Thus, $s_{x}\left(S^{I}\right)=S^{I} * E(x)$, and the lemma follows.

The lemma implies that

$$
E(x) * E(y)=E(x y)
$$

which is clearly equivalent to the claimed result. 
Example 3.19 Taking $F_{n}=\Psi_{n}$, one finds

$$
\begin{gathered}
E_{(3)}(\Psi)=\frac{1}{3} \Psi_{3}=\frac{1}{3}\left[R_{3}-R_{12}+R_{111}\right] \\
E_{(21)}(\Psi)=\frac{1}{6} \Psi^{21}+\frac{1}{3} \Psi^{12}=\frac{1}{6}\left[3 R_{3}-R_{21}+R_{12}-3 R_{111}\right] \\
E_{(111)}(\Psi)=\frac{1}{6} \Psi^{111}=\frac{1}{6}\left[R_{3}+R_{21}+R_{12}+R_{111}\right] .
\end{gathered}
$$

and for $n=4$ :

$$
\begin{gathered}
E_{4}(\Psi)=\frac{1}{4}\left[R_{4}-R_{13}+R_{112}-R_{1111}\right] \\
E_{(31)}(\Psi)=\frac{1}{12}\left[4 R_{4}+R_{31}-3 R_{22}+3 R_{211}+2 R_{13}-R_{121}-2 R_{112}+4 R_{1111}\right] \\
E_{(22)}=\frac{1}{8}\left[R_{4}-R_{31}+R_{22}-R_{211}-R_{13}+R_{121}-R_{112}+R_{1111}\right] \\
E_{(211)}(\Psi)=\frac{1}{12}\left[3 R_{4}+R_{22}-2 R_{211}+2 R_{13}-R_{121}-3 R_{1111}\right] \\
E_{(1111)}(\Psi)=\frac{1}{24}\left[R_{4}+R_{31}+R_{22}+R_{211}+R_{13}+R_{121}+R_{112}+R_{1111}\right] .
\end{gathered}
$$

One can also define analogues of the Eulerian idempotents associated to any series $\left(F_{n}\right)$ of Lie idempotents, by the formula

$$
E_{n}^{[k]}(F)=\sum_{|\lambda|=n, \ell(\lambda)=k} E_{\lambda}(F)
$$

In every case, these idempotents generate an $n$-dimensional commutative subalgebra of $\Sigma_{n}$

Example 3.20 With $F_{n}=\Psi_{n}$, the generating functions of the $E_{n}^{[k]}(\Psi)$ has an interesting expression on the ribbon basis. For $n=4$, we obtain

$$
\begin{gathered}
\sum_{k=1}^{4} x^{k} E_{4}^{[k]}(\Psi)=\frac{1}{24} x(x+1)(x+2)(x+3) R_{4}+\frac{1}{24} x^{2}(x-1)(x+1) R_{31} \\
+\frac{1}{24} x^{2}(x-1)(x+3) R_{22}+\frac{1}{24} x^{2}(x-3)(x-1) R_{211}+\frac{1}{24} x(x-1)(x+2)(x+3) R_{13} \\
+\frac{1}{24} x^{2}(x-1)^{2} R_{121}+\frac{1}{24} x(x-2)(x-1)(x+3) R_{112}+\frac{1}{24} x(x-3)(x-2)(x-1) R_{1111} .
\end{gathered}
$$

A formula for the coefficients will be given in Section 7 .

Other idempotents will be obtained in the sequel, in particular a $q$-deformation of the usual Eulerian idempotents. 


\subsection{Characters of the associated $G L(N)$-modules}

Let $V$ be a finite dimensional vector space over $\mathbb{C}$, and consider the right action of $\mathfrak{S}_{n}$ on the tensor space $T^{n}(V)$, defined as usual by

$$
v_{1} \otimes \cdots \otimes v_{n} \cdot \sigma=v_{\sigma(1)} \otimes \cdots \otimes v_{\sigma(n)} .
$$

As this action commutes with the left action of $G L(V)$ on $T^{n}(V)$, any idempotent $e$ of $\mathbb{C}\left[\mathfrak{S}_{n}\right]$ determines a $G L(V)$-invariant subspace $T^{n}(V) e$.

In particular, any complete set $\left(e_{\lambda}\right)_{\lambda \vdash n}$ of orthogonal idempotents of the descent algebra $\Sigma_{n}$, such as those constructed in Section 3.4, determines a decomposition

$$
T^{n}(V)=\bigoplus_{\lambda \vdash n} V_{\lambda}
$$

where $V_{\lambda}=T^{n}(V) e_{\lambda}$.

In the case where the $e_{\lambda}$ are the Garsia-Reutenauer idempotents $e_{\lambda}=\alpha^{-1}\left(E_{\lambda}(\Phi)\right)$, the characters $L_{\lambda}$ of the $G L(V)$-modules $V_{\lambda}$ are known. Indeed, if $\lambda=\left(1^{m_{1}} 2^{m_{2}} \ldots n^{m_{n}}\right)$, then $V_{\lambda}$ is isomorphic to a product of symmetric powers

$$
S^{m_{1}}\left(L^{1}(V)\right) \otimes S^{m_{2}}\left(L^{2}(V)\right) \otimes \cdots \otimes S^{m_{n}}\left(L^{n}(V)\right)
$$

of homogeneous components $L^{i}(V)$ of the free Lie algebra $L(V)$ over $V$. Thus, as a symmetric function, $L_{\lambda}$ is given by a product of plethysms

$$
L_{\lambda}=h_{m_{1}}\left[\ell_{1}\right] h_{m_{2}}\left[\ell_{2}\right] \cdots h_{m_{n}}\left[\ell_{n}\right]
$$

where $\ell_{n}=\frac{1}{n} \sum_{d \mid n} \mu(d) p_{d}^{n / d}$ is the character of $G L(V)$ in $L^{n}(V)$.

We shall show that the character of $V_{\lambda}$ is always the same, whenever the $e_{\lambda}$ are defined by the construction of section 3.4. More generally, we shall see that in fact, this character only depends on the commutative image of the noncommutative symmetric function $E_{\lambda}=\alpha\left(e_{\lambda}\right)$.

Theorem 3.21 Let $e_{\lambda}^{\prime}$ and $e_{\lambda}^{\prime \prime}$ be two idempotents of $\Sigma_{n}$ such that the associated noncommutative symmetric functions $E_{\lambda}^{\prime}=\alpha\left(e_{\lambda}\right)$ and $E_{\lambda}^{\prime \prime}=\alpha\left(e_{\lambda}^{\prime \prime}\right)$ both have as commutative image the normalized product of power sums $p_{\lambda} / z_{\lambda}$. Set $V_{\lambda}^{\prime}=T^{n}(V) e_{\lambda}^{\prime}$ and $V_{\lambda}^{\prime \prime}=T^{n}(V) e_{\lambda}^{\prime \prime}$. Then, the endomorphism $\phi$ of $T^{n}(V)$ defined by

$$
\phi\left(v_{1} \otimes \cdots \otimes v_{n}\right)=\left(v_{1} \otimes \cdots \otimes v_{n}\right)\left(i d-e_{\lambda}^{\prime}-e_{\lambda}^{\prime \prime}\right)
$$

induces an isomorphism of $G L(V)$-modules between $V_{\lambda}^{\prime}$ and $V_{\lambda}^{\prime \prime}$.

Proof - By construction, it is clear that $\phi$ commutes with the action of $G L(V)$. Also, if $\mathbf{u}^{\prime} \in V_{\lambda}^{\prime}$, then $\mathbf{u}^{\prime}=\mathbf{u}^{\prime} e_{\lambda}^{\prime}$ and $\phi\left(\mathbf{u}^{\prime}\right)=-\mathbf{u}^{\prime} e_{\lambda}^{\prime \prime} \in V_{\lambda}^{\prime \prime}$. Similarly, if $\mathbf{u}^{\prime \prime} \in V_{\lambda}^{\prime \prime}$ then $\phi\left(\mathbf{u}^{\prime \prime}\right) \in V_{\lambda}^{\prime}$. We have to show that $\phi$ is bijective. This will be true if we can prove that $i d-e_{\lambda}^{\prime}-e_{\lambda}^{\prime \prime}$ is invertible in $\Sigma_{n}$, or equivalently that $P=S_{n}-E_{\lambda}^{\prime}-E_{\lambda}^{\prime \prime}$ is invertible for $*$ in $\mathbf{S y m}_{n}$. But we know the eigenvalues of the endomorphisms $L_{P}: F \mapsto P * F$ and $R_{P}: F \mapsto F * P$ (Proposition 3.12). These are the scalar products

$$
\begin{gathered}
t_{\mu}=\left\langle P, p_{\mu}\right\rangle=\left\langle h_{n}-2 \frac{p_{\lambda}}{z_{\lambda}}, p_{\mu}\right\rangle \\
=1-2 \delta_{\lambda \mu}
\end{gathered}
$$

Thus, all the eigenvalues of $L_{P}$ are nonzero, which proves that $\phi$ is an isomorphism. 
Corollary 3.22 Let $\left(e_{\lambda}\right)_{\lambda \vdash n}$ be a complete family of orthogonal idempotents of $\Sigma_{n}$, such that the commutative image of $\alpha\left(e_{\lambda}\right)$ is $p_{\lambda} / z_{\lambda}$. Then the character of $G L(V)$ in $V_{\lambda}$ is the symmetric function

$$
L_{\lambda}=h_{m_{1}}\left[\ell_{1}\right] h_{m_{2}}\left[\ell_{2}\right] \cdots h_{m_{n}}\left[\ell_{n}\right] .
$$

Experimental material suggests that the intertwiners $\pi=i d-e_{\lambda}^{\prime}-e_{\lambda}^{\prime \prime}$ are in fact involutions. The following property seems also to be true:

Conjecture 3.23 Let $\mathcal{E}_{\lambda}=\left\{E_{\lambda}(F) \mid F \in \mathcal{F}\right\}$, where $\mathcal{F}$ is the set of all sequences $\left(F_{n}\right)$ of Lie idempotents, with $F_{n} \in \mathbf{S y m}_{n}$. Then, the convex hull $\mathcal{A}_{\lambda}$ of $\mathcal{E}_{\lambda}$ is an affine space, and all its elements are idempotents.

\subsection{Dimensions of the quasi-ideals $e_{\lambda} \Sigma_{n} e_{\mu}$}

Let $\left(e_{\lambda}\right)_{\lambda \vdash n}$ be any complete set of orthogonal idempotents of $\Sigma_{n}$, such that, as in the preceding section, the commutative image of each $\alpha\left(e_{\lambda}\right)$ is equal to $p_{\lambda} / z_{\lambda}$. We have then a decomposition of $\Sigma_{n}$ as a direct sum

$$
\Sigma_{n}=\bigoplus_{\lambda, \mu \vdash n} e_{\lambda} \Sigma_{n} e_{\mu}
$$

and the problem is to compute the dimensions of the subspaces $e_{\lambda} \Sigma_{n} e_{\mu}$. For the GarsiaReutenauer idempotents, this problem is solved in [11]. This is sufficient to deal with the general case. Indeed, let $\left(e_{\lambda}^{\prime}\right)$ be a second family satifying the same condition on the commutative images. Then, using the intertwiners constructed in the preceding section, we see that

$$
\left(i d-e_{\lambda}-e_{\lambda}^{\prime}\right) e_{\lambda} \Sigma_{n} e_{\mu}\left(i d-e_{\mu}-e_{\mu}^{\prime}\right)=e_{\lambda}^{\prime} e_{\lambda} \Sigma_{n} e_{\mu} e_{\mu}^{\prime} \subseteq e_{\lambda}^{\prime} \Sigma_{n} e_{\mu}^{\prime}
$$

is in fact equal to $e_{\lambda}^{\prime} \Sigma_{n} e_{\mu}^{\prime}$, since the left and right multiplication by the elements $i d-e-e^{\prime}$ are one-to-one.

Theorem 3.24 Let $\left(e_{\lambda}\right)$ be a complete set of orthogonal idempotents of $\Sigma_{n}$ satisfying the above conditions on the commutative images. Then, the dimension of the subspace $e_{\lambda} \Sigma_{n} e_{\mu}$ is equal to the number of multisets of primitive circular words of shape $\lambda$ and type $\mu$ (see [11], [3] or [33] for this terminology).

Proof - This follows from the above considerations, combined with Theorem 5.4. of [11]. For the sake of completeness, we give an alternative proof of this last result in terms of noncommutative symmetric functions. Suppose $E_{\lambda}=E_{\lambda}(\Phi)$, and for a composition $I$ of $n$, consider the Lie polynomial

$$
P_{I}=\frac{\Phi_{n}}{n} * \Phi^{I} \in L(\Phi)=L(\Psi)
$$

Then,

$$
E_{\mu} * \Phi^{J}=\sum_{I \in \mathfrak{S}(\mu)} \frac{\Phi^{I}}{\pi(I)} * \Phi^{J}
$$


where, as above, $\mathfrak{S}(\mu)$ denotes the set of all distinct reorderings of $\mu$, and for $I=$ $\left(i_{1}, \ldots, i_{s}\right)$

$$
\begin{gathered}
\frac{\Phi^{I}}{\pi(I)} * \Phi^{J}=\mu_{s}\left[\frac{\Phi_{i_{1}}}{i_{1}} \otimes \cdots \otimes \frac{\Phi_{i_{s}}}{i_{s}} * \sum_{J_{1}, \ldots, J_{s}}\left\langle J, J_{1} \sqcup \cdots \sqcup J_{s}\right\rangle \Phi^{J_{1}} \otimes \cdots \otimes \Phi^{J_{s}}\right] \\
=\sum_{\substack{J_{1}, \ldots J_{s} \\
\left|J_{k}\right|=i_{k}}}\left\langle J, J_{1} \sqcup \cdots \sqcup J_{s}\right\rangle P_{J_{1}} P_{J_{2}} \cdots P_{J_{s}} .
\end{gathered}
$$

Thus,

$$
\begin{gathered}
E_{\mu} * \Phi^{J}=\sum_{\left(\left|J_{1}\right|, \ldots,\left|J_{s}\right|\right) \in \mathfrak{S}(\mu)}\left\langle J, J_{1} \sqcup \ldots \sqcup J_{s}\right\rangle P_{J_{1}} P_{J_{2}} \cdots P_{J_{s}} \\
=s ! \sum_{\left(\left|J_{1}, \ldots,\right| J_{s} \mid\right) \in \mathfrak{S}(\mu)}\left\langle J, J_{1} \sqcup \ldots \sqcup J_{s}\right\rangle\left(P_{J_{1}}, P_{J_{2}}, \ldots, P_{J_{s}}\right)
\end{gathered}
$$

where $\left(F_{1}, F_{2}, \ldots, F_{s}\right)=\frac{1}{s !} \sum_{\sigma \in \mathfrak{S}_{s}} F_{\sigma(1)} \cdots F_{\sigma(s)}$ denotes the symmetrized product. Thus, $E_{\mu} * \Phi^{J}$ belongs to the space $S^{\mu} L(\Phi)$, spanned by the symmetrized products of type $\mu$ of Lie polynomials in the $\Phi_{i}$.

Consider now the products $\Phi^{J} * E_{\lambda}$. Using once more the splitting formula for internal products, we see that for $J=\left(j_{1}, \ldots, j_{m}\right)$,

$$
\Phi^{J} * E_{\lambda}=\sum_{\lambda^{1} \vee \cdots \vee \lambda^{m}=\lambda}\left(\Phi_{j_{1}} * E_{\lambda^{1}}\right) \cdots\left(\Phi_{j_{m}} * E_{\lambda^{m}}\right)
$$

where $\alpha \vee \beta$ denotes the reordered concatenation of two partitions $\alpha$ and $\beta$. Indeed, the generating series $E(x)$ being grouplike, we have

$$
\Delta^{m} E_{\lambda}=\sum_{\lambda^{1} \vee \cdots \vee \lambda^{m}=\lambda} E_{\lambda^{1}} \otimes \cdots \otimes E_{\lambda^{m}}
$$

Moreover, we know that $\Phi_{j} * E_{\lambda}=0$ for $\lambda \neq(j)$ (Lemma 3.14). Thus,

$$
\Phi^{J} * E_{\lambda}= \begin{cases}0 & \text { if } J \notin \mathfrak{S}(\lambda) \\ \Phi^{J} & \text { if } J \in \mathfrak{S}(\lambda)\end{cases}
$$

This shows that for any composition $J, E_{\mu} * \Phi^{J} * E_{\lambda}$ belongs to the subspace $\left[S^{\mu} L(\Phi)\right]_{\lambda}$, which is defined as the intersection of the subspace $S^{\mu} L(\Phi)$ with the subspace

$$
\operatorname{Sym}_{\lambda}(\Phi)=\operatorname{Vect}\left\langle\Phi^{K} \mid K \in \mathfrak{S}(\lambda)\right\rangle \text {. }
$$

To conclude, we know that the image of $L_{E_{\mu}}: F \mapsto E_{\mu} * F$ is the whole $S^{\mu}(\Phi)$, since $\sum_{\mu \vdash n} E_{\mu}=S_{n}$, and $\mathbf{S y m}_{n}=\left.\bigoplus_{\mu \vdash n} S^{\mu} L(\Phi)\right|_{n}$. Hence, $E_{\mu} * \mathbf{S y m}_{n} * E_{\lambda}=\left[S^{\mu} L(\Phi)\right]_{\lambda}$, and thus the dimension of $e_{\lambda} \Sigma_{n} e_{\mu}$ is equal to the dimension of $=\left[S^{\mu} L(\Phi)\right]_{\lambda}$, which is clearly equal to the number of multisets of circular words of shape $\lambda$ and weight $\mu$. 


\section{Sums, differences and products of alphabets}

When we need to use simultaneously several copies of the algebra of noncommutative symmetric functions, it is convenient to distinguish between them by labels $A, B, C, \ldots$. attributed to their elements, e.g. $\Lambda^{I}(A), R_{I}(B)$ and so on. It is even more convenient to interpret these labels as virtual arguments (informally called alphabets) of the noncommutative symmetric functions. In the commutative case, these arguments can be interpreted as elements of some $\lambda$-ring, by identifying a set of indeterminates with the formal sum of its elements. Thus, in the commutative case, one can consider sums, differences, products, and even more general functions of alphabets.

It is unlikely that noncommutative symmetric functions can be interpreted as operators for a good notion of noncommutative $\lambda$-ring, but it is still possible and useful to define symmetric functions of sums and differences of noncommutative alphabets. As for the product, the proper analog seems to be the product of a commutative ordered alphabet with a noncommutative alphabet.

In the case of commutative ordered alphabets, which are the natural arguments of quasi-symmetric functions [15], the notions of sum, product and difference correspond to the two coproducts and antipode of the algebra of quasi-symmetric functions. Remark that noncommutative alphabets also have to be regarded as totally ordered. The noncommutative symmetric functions of an infinite ordered set of noncommuting letters $A=\left\{a_{1}<a_{2}<\ldots\right\}$ can be realized by defining

$$
\sigma(A ; t)=\prod_{k \geq 1}^{\leftarrow}\left(1-t a_{k}\right)^{-1}
$$

but it is more convenient to work at the abstract level, with virtual alphabets.

A different noncommutative extension of the $\lambda$-ring formalism appears in [30].

\subsection{Sums of alphabets}

In the commutative case, a symmetric function $f(X+Y)$ of a sum of alphabets can be identified with its coproduct $\Delta f$ by means of the standard identification $u(X) v(Y) \equiv$ $u \otimes v$. In the noncommutative case, these two notions bifurcate.

Definition 4.1 Let $A$ and $B$ be two noncommutative alphabets. The complete symmetric functions $S_{n}(A \oplus B ; t)$ of the alphabet $A \oplus B$ are defined by the generating series

$$
\sigma(A \oplus B ; t):=\sum_{n \geq 0} S_{n}(A \oplus B) t^{n}=\sigma(A ; t) \sigma(B ; t)
$$

In the noncommutative case, the sum $A \oplus B$ of two virtual alphabets $A$ and $B$ is an ordered sum. It follows that $A \oplus B \neq B \oplus A$.

The additivity of the Adams operators of a $\lambda$-ring is replaced by the following identity.

Proposition 4.2 Let $A$ and $B$ be two noncommutative alphabets. Then,

$$
\psi(A \oplus B ; t)=\sigma(B ; t)^{-1} \psi(A ; t) \sigma(B ; t)+\psi(B ; t)
$$


Proof - According to our definitions,

$$
\begin{gathered}
\psi(A \oplus B ; t)=\sigma(A \oplus B ; t)^{-1} \frac{d}{d t} \sigma(A \oplus B ; t) \\
=\sigma(B ; t)^{-1} \sigma(A ; t)^{-1}\left(\left(\frac{d}{d t} \sigma(A ; t)\right) \sigma(B ; t)+\sigma(A ; t) \frac{d}{d t} \sigma(B ; t)\right),
\end{gathered}
$$

which is the required formula.

This implies that $\Psi_{n}(A \oplus B)$ differs from $\Psi_{n}(A)+\Psi_{n}(B)$ by a nontrivial Lie polynomial in the $\Psi_{i}(A), \Psi_{j}(B)$. This property is in fact true of the symmetric functions corresponding to any sequence of Lie idempotents:

Proposition 4.3 Let, for all $n \geq 1, \pi_{n}$ be a Lie idempotent of $\Sigma_{n}$, and $F_{n}=\alpha\left(\pi_{n}\right) \in$ $\mathrm{Sym}_{n}$. Then,

$$
F_{n}(A \oplus B)=F_{n}(A)+F_{n}(B)+l^{\geq 2}(\pi)
$$

where $l^{\geq 2}(\pi)$ is an element of the Lie ideal $[L(F(A), F(B)), L(F(A), F(B))]$.

Proof - According to Theorem 3.1, it is sufficient to check this property for one given family of Lie idempotents. Taking $F_{n}=\Phi_{n}$, we have

$$
\Phi(A \oplus B)=\log (\sigma(A \oplus B))=\log (\sigma(A) \sigma(B))=\log (\exp (\Phi(A)) \exp (\Phi(B))),
$$

and the conclusion follows from the Baker-Campbell-Hausdorff formula.

Example 4.4 Using Proposition 4.2, we find

$$
\begin{gathered}
\Psi_{2}(A \oplus B)=\Psi_{2}(A)+\Psi_{2}(B)+\left[\Psi_{1}(B), \Psi_{1}(A)\right], \\
\Psi_{3}(A \oplus B)=\Psi_{3}(A)+\Psi_{3}(B)+\left[\Psi_{2}(B), \Psi_{1}(A)\right]+\left[\Psi_{1}(B), \Psi_{2}(A)\right] \\
+\frac{1}{2}\left[\left[\Psi_{1}(B), \Psi_{1}(A)\right], \Psi_{1}(A)\right] .
\end{gathered}
$$

\subsection{Differences of alphabets}

Let us first recall the definition of the quasi-symmetric functions of the difference of two totally ordered commutative alphabets [24]. Let $F$ and $G$ be two quasi-symmetric functions, and $X, Y$ be two ordered alphabets. Identify $F \otimes G$ with $F(X) G(Y)$. Then, the coproduct $\gamma$ of $[24]$ is defined by

$$
\gamma(F)=F(X \hat{+} Y)
$$

where $X \hat{+} Y$ denotes the ordered sum of $X$ and $Y$. Let also $P$ be the permutation operator $P(F \otimes G)=G \otimes F$.

Definition 4.5 Let $X$ and $Y$ be two totally ordered commutative alphabets. The quasisymmetric functions of the difference $X-Y$ are defined by

$$
F(X-Y)=(\nu \otimes 1) \circ P \circ \gamma(F),
$$

where $\nu$ is the antipode of QSym, defined on the basis $F_{I}$ by $\nu\left(F_{I}\right)=(-1)^{|I|} F_{I^{\sim}}$. 
Note 4.6 In other words, $X-Y$ is the virtual ordered alphabet $(-Y) \hat{+} X$.

In order that the difference operation on noncommutative alphabets be compatible with the product operation to be defined in the next section, we have to define it as follows:

Definition 4.7 Let $A$ and $B$ be two noncommutative alphabets. The complete symmetric functions of the alphabet $A-B$ are defined by the generating series

$$
\sigma(A-B ; t)=\sum_{n \geq 0} S_{n}(A-B) t^{n}:=\lambda(B ;-t) \sigma(A ; t) .
$$

Thus, $A-B=(-B) \oplus A$, as in Definition 4.5.

Definition 4.7 can be rewritten as

$$
S_{n}(A-B)=\sum_{i=0}^{n}(-1)^{i} \Lambda_{i}(B) S_{n-i}(A) .
$$

Inverting relation (44), we also obtain

$$
\lambda(A-B ; t)=\lambda(A ; t) \sigma(B ;-t),
$$

so that

$$
\omega\left(S_{n}(A-B)\right)=\Lambda_{n}(A-B)=(-1)^{n} S_{n}(B-A) .
$$

In particular, we have as in the commutative case

$$
S_{n}(-A)=(-1)^{n} \Lambda_{n}(A) .
$$

\subsection{Products of alphabets}

The product of two noncommutative alphabets does not seem to lead to interesting computations. On the other hand, the product of a commutative ordered alphabet with a noncommutative alphabet is an important notion, which accounts for the duality between noncommutative symmetric functions and quasi-symmetric functions ( $c f .[24,12])$ and gives rise to noncommutative analogs of Cauchy-type identities.

Definition 4.8 Let $X$ be a totally ordered commutative alphabet and let $A$ be a noncommutative alphabet. The complete symmetric functions $S_{n}(X A)$ of the (noncommutative) alphabet $X A$ are defined by the generating series

$$
\sigma(X A ; t)=\sum_{n \geq 0} S_{n}(X A) t^{n}:=\prod_{x \in X} \sigma(A ; x t)
$$

the product being taken relatively to the total ordering of $X$.

This means that

$$
\begin{aligned}
S_{n}(X A)= & \sum_{x_{j_{1}}<\ldots<x_{j_{k}},|I|=n} x_{j_{1}}^{i_{1}} \ldots x_{j_{k}}^{i_{k}} S_{i_{1}} \ldots S_{i_{k}} \\
= & \sum_{|I|=n} M_{I}(X) S^{I}(A)
\end{aligned}
$$

where $M_{I}(X)$ is the quasi-monomial function of the alphabet $X$ indexed by $I$. 
Note 4.9 Applying $\omega$ to (50) gives the formula

$$
\Lambda_{n}(X A)=\sum_{|I|=n} M_{I}(X) \Lambda^{\bar{I}}(A)
$$

Formula (50) is a particular case of the following identity, which expresses the duality between QSym and Sym, and appears to be the proper analog of the Cauchy-type identities of the commutative theory:

Proposition 4.10 (Cauchy formula) Let $\left(V_{I}(A)\right)$ be any homogeneous basis of $\mathbf{S y m}$ and let $\left(U_{I}(X)\right)$ be its dual basis in QSym. Then one has

$$
S_{n}(X A)=\sum_{|I|=n} U_{I}(X) V_{I}(A)
$$

Proof - Decomposing $S^{I}(A)$ in the basis $\left(V_{I}(A)\right)$, we can write

$$
S^{I}(A)=\sum_{|K|=n} c_{I, K} V_{K}(A)
$$

It follows then from (50) that

$$
S_{n}(X A)=\sum_{|I|=n} M_{I}(X)\left(\sum_{|K|=n} c_{I, K} V_{K}(A)\right)=\sum_{|K|=n}\left(\sum_{|I|=n} c_{I, K} M_{I}(X)\right) V_{K}(A)
$$

But, for two compositions $I$ and $K$, one has

$$
\left(U_{K}(X) \mid S^{I}(A)\right)=\sum_{L} c_{I, L}\left(U_{K}(X) \mid V_{L}(A)\right)=c_{I, K}
$$

so that

$$
U_{K}(X)=\sum_{|I|=n} c_{I, K} M_{I}(X)
$$

and reporting this relation in (53) gives the desired formula.

Taking $V_{I}=R_{I}$, the formula reads as

$$
S_{n}(X A)=\sum_{|I|=n} F_{I}(X) R_{I}(A)
$$

where $\left(F_{I}(X)\right)$ is the family of quasi-ribbon functions of QSym.

Proposition 4.11 Let $X$ be a totally ordered commutative alphabet, let $\bar{X}$ denote the opposite alphabet (i.e. $\bar{X}$ is $X$ equipped with the opposite total order) and let $A$ be a noncommutative alphabet. Then,

$$
F(\bar{X} A)=(F(X A))^{*}
$$

for any noncommutative symmetric function $F$. 
Proof - It is sufficient to check the formula for complete functions, and since $*$ is an antiautomorphism,

$$
\sigma(\bar{X} A ; t)=\prod_{x \in \bar{X}} \sigma(A ; x t)=\left(\prod_{x \in X} \sigma(A ; x t)\right)^{*}=(\sigma(X A ; t))^{*} .
$$

In particular, Proposition 4.11 and formula (50) give the (obvious) relation

$$
M_{I}(\bar{X})=M_{\bar{I}}(X)
$$

One can now check the compatibility of the product of alphabets given by Definition 4.8 with the product of ordered alphabets.

Proposition 4.12 Let $X, Y$ be two totally ordered commutative alphabets and let $A$ be a noncommutative alphabet. Then,

$$
F((X \hat{\times} Y) A)=F(X(Y A))
$$

for every noncommutative symmetric function $F$.

Proof - It is sufficient to check the formula for complete functions. In this case,

$$
\sigma(X(Y A) ; t)=\prod_{x \in X} \sigma(Y A ; x t)=\prod_{x \in X}\left(\prod_{y \in Y} \sigma(A ; x y t)\right)=\sigma((X \hat{\times} Y) A ; t) .
$$

We can also connect products of alphabets and duality in the following way.

Proposition 4.13 Let $X$ and $A$ be respectively a totally ordered commutative and a noncommutative alphabet in duality with each other, which means that the dual of $\mathbf{S y m}(A)$ is identified with $Q \operatorname{Sym}(X)$, and write $(P(X) \mid Q(A))$ for the pairing between the two spaces. Let $Y$ be another totally ordered commutative alphabet. Then,

$$
(F(X) \mid G(Y A))=(F(Y \hat{\times} X) \mid G(A)),
$$

for any quasi-symmetric function $F$ and any noncommutative symmetric function $G$.

Proof - Let us show first that (57) holds for $F=M_{K}$ and $G=S_{n}$. In this case, according to (50), one has

$$
\begin{gathered}
\left(M_{K}(X) \mid S_{n}(Y A)\right)=\left(M_{K}(X) \mid \sum_{|I|=n} M_{I}(Y) S^{I}(A)\right) \\
=\sum_{|I|=n} M_{I}(Y)\left(M_{K}(X) \mid S^{I}(A)\right)=M_{K}(Y) .
\end{gathered}
$$

On the other hand, one can write

$$
M_{K}(Y \hat{\times} X)=\sum_{I, J} a_{I J} M_{I}(Y) M_{J}(X)
$$


with some scalar coefficients $a_{I J}$. It follows that

$$
\left(M_{K}(Y \hat{\times} X) \mid S_{n}(A)\right)=\sum_{I} a_{I n} M_{I}(Y)=\sum_{I}\left(M_{K}(Y \hat{\times} X) \mid S^{I} \otimes S_{n}\right) M_{I}(Y) .
$$

By duality, one has

$$
\begin{gathered}
\left(M_{K}(Y \hat{\times} X) \mid S^{I} \otimes S_{n}\right)=\left(\delta\left(M_{K}\right) \mid S^{I} \otimes S_{n}\right)=\left(M_{K} \mid S^{I} * S_{n}\right) \\
=\left(M_{K} \mid S^{I}\right)=\left\{\begin{array}{ll}
1 & \text { if } I=K \\
0 & \text { if } I \neq K
\end{array} .\right.
\end{gathered}
$$

Reporting this result in the last formula, we obtain

$$
\left(M_{K}(Y \hat{\times} X) \mid S_{n}(A)\right)=M_{K}(Y) .
$$

Hence,

$$
\left(M_{K}(X) \mid S_{n}(Y A)\right)=\left(M_{K}(Y \hat{\times} X) \mid S_{n}(A)\right)=M_{K}(Y)
$$

which is (57) in the case under consideration. We now deal with the general case. Note that by linearity, we may suppose that $F=M_{K}$ and $G=S^{I}$. In this case,

$$
\begin{gathered}
\left(M_{K}(X) \mid S^{I}(Y A)\right)=\left(M_{K}\left(X_{1} \hat{+} \ldots \hat{+} X_{r}\right) \mid S_{i_{1}}(Y A) \otimes \ldots \otimes S_{i_{r}}(Y A)\right) \\
=\sum_{P_{1} \ldots P_{r}=K}\left(M_{P_{1}}\left(X_{1}\right) \otimes \ldots \otimes M_{P_{r}}\left(X_{r}\right) \mid S_{i_{1}}(Y A) \otimes \ldots \otimes S_{i_{r}}(Y A)\right) \\
\quad=\sum_{P_{1} \ldots P_{r}=K}\left(M_{P_{1}}\left(X_{1}\right) \mid S_{i_{1}}(Y A)\right) \ldots\left(M_{P_{r}}\left(X_{r}\right) \mid S_{i_{r}}(Y A)\right),
\end{gathered}
$$

where the quasi-symmetric functions of $X_{i}$ are in duality with the $i$-th factor of the tensor product. Using (58), we get

$$
\left(M_{K}(X) \mid S^{I}(Y A)\right)=\sum_{P_{1} \ldots P_{r}=K}\left(M_{P_{1}}\left(Y \hat{\times} X_{1}\right) \mid S_{i_{1}}(A)\right) \ldots\left(M_{P_{r}}\left(Y \hat{\times} X_{r}\right) \mid S_{i_{r}}(A)\right)
$$

and, backtracking these computations, it is easy to see that this last expression is equal to $\left(M_{K}\left(Y \hat{\times} X \mid S^{I}(A)\right)\right.$.

Note 4.14 Observe that the proof of Proposition 4.13 gives also the formula

$$
\left(M_{K}(X) \mid S^{I}(Y A)\right)=\sum_{P_{1} \ldots P_{\ell(I)}=K} M_{P_{1}}(Y) \ldots M_{P_{\ell(I)}}(Y)=M_{K}(\ell(I) Y)
$$

where $\ell(I) Y$ denotes the alphabet $Y \hat{+} \ldots \hat{+} Y$, this sum involving $\ell(I)$ copies of $Y$.

One can now connect the sum and difference of noncommutative alphabets with the product of alphabets as defined in this section.

Proposition 4.15 Let $X$ and $Y$ be two totally ordered commutative alphabets and let $A$ be a noncommutative alphabet. Then,

$$
F((X \hat{+} Y) A)=F(X A \oplus Y A) \text { and } F((X-Y) A)=F(X A-Y A) .
$$

for every noncommutative symmetric function $F$. 
Proof - It is sufficient to check the relation for complete functions. In this case, one has

$$
\begin{gathered}
S_{n}((X \hat{+} Y) A)=\sum_{|I|=n} M_{I}(X \hat{+} Y) S^{I}(A)=\sum_{|I|=n}\left(\sum_{P Q=I} M_{P}(X) M_{Q}(Y)\right) S^{I}(A) \\
=\sum_{|P|+|Q|=n} M_{P}(X) M_{Q}(Y) S^{P}(A) S^{Q}(A) .
\end{gathered}
$$

It follows that

$$
\begin{aligned}
S_{n}((X \hat{+} Y) A) & =\sum_{p+q=n}\left(\sum_{|P|=p} M_{P}(X) S^{P}(A)\right)\left(\sum_{|Q|=q} M_{Q}(Y) S^{Q}(A)\right) \\
& =\sum_{p+q=n} S_{p}(X A) S_{q}(Y A)=S_{n}(X A \oplus Y A)
\end{aligned}
$$

as required. The second formula is a consequence of the first one.

Note 4.16 Let $X$ be a totally ordered commutative alphabet and let $A, B$ be two noncommutative alphabets. Observe that, in general, one has

$$
F(X(A \oplus B)) \neq F(X A \oplus X B)
$$

For instance,

$$
S_{2}(X(A \oplus B))-S_{2}(X A \oplus X B)=M_{11}(X)\left[S_{1}(B), \Lambda_{1}(A)\right] .
$$

In fact, one has for complete symmetric functions

$$
\begin{gathered}
\sigma(X A \oplus X B ; t)-\sigma(X(A \oplus B) ; t) \\
=\left(\prod_{x \in X} \sigma(A ; x t)\right)\left(\prod_{x \in X} \sigma(B ; x t)\right)-\prod_{x \in X}(\sigma(A ; x t) \sigma(B ; x t)) .
\end{gathered}
$$

This expression is clearly not equal to zero in general. However it is zero when $\mathbf{S y m}(A)$ commutes with $\operatorname{Sym}(B)$, i.e. when one has $\left[S_{i}(A), S_{j}(B)\right]=0$ for every $i, j \geq 0$. It follows that relation (60) holds in this special case.

The transformation $F(A) \mapsto F(X A)$, where $X$ and $A$ are allowed to be virtual, can be performed by means of a "reproducing kernel identity":

Theorem 4.17 Let $X$ be a (virtual) commutative ordered alphabet, and $A$ be a virtual noncommutative alphabet. Then, for any noncommutative symmetric function $F$,

$$
F(X A)=F(A) * \sigma(X A ; 1)
$$

The theorem is a consequence of the following two lemmas.

Lemma 4.18 Let $X, Y$ be two totally ordered commutative alphabets and let $A$ be a noncommutative alphabet. Then, for every $n \geq 0$, one has

$$
\sigma(X A ; 1) * \sigma(Y A ; 1)=\sigma((X \hat{\times} Y) A ; 1)
$$


Proof - Using the fact that $\sigma(Y A ; t)$ is a group-like element for $\Delta$, one gets

$$
\sigma(X A ; 1) * \sigma(Y A ; 1)=\mu_{X}\left[\bigotimes_{x \in X} \sigma(A ; x) * \sigma(Y A ; 1)^{\otimes X}\right] .
$$

But it is easy to see that

$$
\sigma(A ; x) * \sigma(Y A ; 1)=\sigma(Y A ; x)
$$

for every $x \in X$. Hence,

$$
\sigma(X A ; 1) * \sigma(Y A ; 1)=\overleftarrow{\prod_{x \in X}} \sigma(Y A ; x)=\sigma(X(Y A) ; 1)=\sigma((X \hat{\times} Y) A ; 1)
$$

according to Proposition 4.12.

Lemma 4.19 Let $X, Y$ be two totally ordered commutative alphabets and let $A$ be a noncommutative alphabet. Then, for any noncommutative symmetric function $F$,

$$
F(X A) * \sigma(Y A ; 1)=F((X \hat{\times} Y) A) .
$$

Proof - It is sufficient to check the formula for $F=S^{I}$. Lemma 4.18 shows the required identity for $I=(n)$, and in the general case, with $I=\left(i_{1}, \ldots, i_{r}\right)$, one has

$$
S^{I}(X A) * \sigma(Y A)=\mu_{r}\left(S_{i_{1}}(X A) \otimes \ldots \otimes S_{i_{r}}(X A) * \sigma(Y A)^{\otimes r}\right)=S^{I}((X \hat{\times} Y) A),
$$

using again Lemma 4.18.

The most useful version of these formulas is the following:

Corollary 4.20 Let $\left(V_{I}\right)$ be any homogeneous basis of $\mathbf{S y m}$, and let $\left(U_{I}\right)$ be its dual basis in the space of quasi-symmetric funtions. Then, for any $F \in \mathbf{S y m}$, one has

$$
F(X A)=\sum_{I} U_{I}(X)\left(F * V_{I}\right)(A) .
$$


The first nontrivial case of a difference of commutative ordered alphabets arises when $X$ and $Y$ are reduced to one element. It is convenient, by analogy with some classical commutative calculations, to take $X=\{1\}$ and $Y=\{q\}$. The commutative transformation $A \mapsto(1-q) A$ is of fundamental importance in the theory of Hall-Littlewood functions (cf. [21]). It is explicitely used in the computation of the character tables of finite linear groups and of Hecke algebras of type $A_{n}$ (see [31] for a $\lambda$-ring version of this last application). In the noncommutative case, this transformation is related to the $q$-bracket operation $[a, b]_{q}=a b-q b a$.

\subsection{Complete functions}

According to the definitions of Section 4, we can describe the complete symmetric functions of the alphabet $(1-q) A$ by their generating series as follows.

Definition 5.1 The generating series of the complete symmetric functions $S_{n}((1-q) A)$ of the alphabet $(1-q) A$ is given by

$$
\sigma((1-q) A) ; t):=\sum_{n \geq 0} S_{n}((1-q) A) t^{n}=\sigma(A ; q t)^{-1} \sigma(A ; t)=\lambda(A ;-q t) \sigma(A ; t) .
$$

The functions $S_{n}((1-q) A)$ are clearly noncommutative analogs of commutative complete symmetric functions of the alphabet $(1-q) A$ (in the sense of $\lambda$-rings).

We first give an expression of $S_{n}((1-q) A)$ in terms of ribbon Schur functions. To this purpose, consider the noncommutative symmetric function defined by

$$
\Theta_{n}(q)=\sum_{i=0}^{n-1}(-q)^{i} R_{1^{i}, n-i}
$$

for $n \geq 1$. Then, one can state :

\section{Proposition 5.2}

$$
S_{n}((1-q) A)=\left\{\begin{array}{cl}
(1-q) \Theta_{n}(q) & \text { if } n \geq 1 \\
1 & \text { if } n=0
\end{array} .\right.
$$

Proof - According to Definition 5.1,

$$
S_{n}((1-q) A)=\sum_{i=0}^{n}(-q)^{i} \Lambda_{i}(A) S_{n-i}(A) .
$$

For $0<i<n$, one can also write

$$
\Lambda_{i}(A) S_{n-i}(A)=R_{1^{i}}(A) R_{n-i}(A)=R_{1^{i}, n-i}+R_{1^{i-1}, n-i+1} .
$$

Reporting this identity in the defining relation of $S_{n}((1-q) A)$, we get

$$
S_{n}((1-q) A)=R_{n}+(-q)^{n} R_{1^{n}}+\sum_{i=1}^{n-1}(-q)^{i}\left(R_{1^{i}, n-i}+R_{1^{i-1}, n-i+1}\right)
$$




$$
=(1-q) \sum_{i=0}^{n-1}(-q)^{i} R_{1^{i}, n-i}
$$

Formula (65) shows that $\sigma((1-q) A ; t)$ is the product of two group-like series for the coproduct $\Delta$. Hence $\sigma((1-q) A ; t)$ is also a group-like element for $\Delta$. This means that

$$
\Delta\left(S_{n}((1-q) A)\right)=\sum_{i=0}^{n} S_{i}((1-q) A) \otimes S_{n-i}((1-q) A) .
$$

This last formula can be equivalently rewritten as

$$
\Delta\left(\Theta_{n}(q)\right)=1 \otimes \Theta_{n}(q)+\Theta_{n}(q) \otimes 1+(1-q)\left(\sum_{i=1}^{n-1} \Theta_{i}(q) \otimes \Theta_{n-i}(q)\right) .
$$

One can also compute the image by $\omega$ of $S_{n}((1-q) A)$.

Proposition 5.3 For every $n \geq 1$, one has

$$
\omega\left(S_{n}((1-q) A)\right)=(-q)^{n} S_{n}((1-1 / q) A) .
$$

Proof - One has

$$
\omega\left(\Theta_{n}(q)\right)=\sum_{i=0}^{n-1}(-q)^{i} \omega\left(R_{1^{i}, n-i}\right)=\sum_{i=0}^{n-1}(-q)^{i} R_{1^{n-i-1}, i+1}=(-q)^{n-1} \Theta_{n}(1 / q) .
$$

\section{$5.2 q$-Newton formulas and consequences}

It is interesting to observe that $\Theta_{n}(q)$ can be considered under two different aspects. Indeed, it interpolates between $S_{n}(q=0)$ and $\Psi_{n}(q=1)$. Thus it shares properties of complete functions and of power sums of the first kind. We shall rather concentrate in this section on the power-sum-like aspects.

Let us introduce the generating series $\vartheta(t)$ defined by

$$
\vartheta(t):=\sum_{n \geq 1} \Theta_{n}(q) t^{n-1}=\frac{\sigma((1-q) A ; t)-1}{(1-q) t}
$$

and consider the $q$-derivative $\sigma_{q}^{\prime}(t)$ defined by

$$
\sigma_{q}^{\prime}(t):=\frac{\sigma(t)-\sigma(q t)}{(1-q) t}=\sum_{n \geq 1}[n]_{q} S_{n}(A) t^{n-1},
$$

where $[n]_{q}$ denotes the $q$-integer $1+q+\ldots+q^{n-1}$. The generating series $\vartheta(t)$ is given by the following proposition: 
Proposition 5.4 One has

$$
\vartheta(t)=\lambda(A ;-q t) \sigma_{q}^{\prime}(A ; t)
$$

Proof — Since by definition

$$
\vartheta(t)=\frac{1}{1-q} \frac{\sigma((1-q) A ; t)-1}{t} .
$$

it follows from (65) that

$$
\vartheta(t)=\frac{1}{1-q} \frac{\lambda(A ;-q t) \sigma(A ; t)-1}{t}=\lambda(A ;-q t) \frac{1}{1-q} \frac{\sigma(A ; t)-\sigma(A ; q t)}{t}
$$

and it is easy to see that

$$
\frac{1}{1-q} \frac{\sigma(A ; t)-\sigma(A ; q t)}{t}=\sum_{n \geq 1} \frac{1-q^{n}}{1-q} t^{n-1}=\sigma_{q}^{\prime}(t) .
$$

Formula (73) gives a $q$-analog of Newton's formulas. Indeed, it says that

$$
\sigma_{q}^{\prime}(t)=\sigma(A ; q t) \vartheta(t)
$$

which can be equivalently expressed as

$$
[n]_{q} S_{n}(A)=\sum_{i=0}^{n-1} q^{i} S_{i}(A) \Theta_{n-i}(q) .
$$

Using rather $S_{n}((1-q) A)$, we also get

$$
\left(1-q^{n}\right) S_{n}(A)=\sum_{i=0}^{n-1} q^{i} S_{i}(A) S_{n-i}((1-q) A) .
$$

These identities also give quasi-determinantal formulas expressing $\Theta_{n}(q)$ or $S_{n}((1-q) A)$ in terms of the complete functions $S_{n}(A)$.

Proposition 5.5 For every $n \geq 1$, one has

$$
\Theta_{n}(q)=\left|\begin{array}{ccccc}
S_{1}(A) & S_{0}(A) & 0 & \ldots & 0 \\
{[2]_{q} S_{2}(A)} & q S_{1}(A) & S_{0}(A) & \ldots & 0 \\
{[3]_{q} S_{3}(A)} & q^{2} S_{2}(A) & q S_{1}(A) & \ldots & 0 \\
\vdots & \vdots & \vdots & \vdots & \vdots \\
{\left[[n]_{q} S_{n}(A)\right]} & q^{n-1} S_{n-1}(A) & q^{n-2} S_{n-2}(A) & \ldots & q S_{1}(A)
\end{array}\right|,
$$


Proof - These formulas are direct consequences of formula (75).

The expression of $S_{n}$ on the basis $\Theta^{I}(q)$ can also be obtained by solving the $q$ differential equation (74) in terms of iterated $q$-integrals, i.e.

$$
\sigma(t)=1+\int_{0}^{t} d_{q} t_{1} \vartheta\left(t_{1}\right)+\int_{0}^{t} d_{q} t_{1} \int_{0}^{t_{1}} d_{q} t_{2} \vartheta\left(q t_{2}\right) \vartheta\left(t_{1}\right)+\cdots
$$

\subsection{The $q$-bracket}

The symmetric function $\Theta_{n}(q)$ corresponds to an interesting element of the descent algebra. Indeed, it is easy to see that the image of $\Theta_{n}(q)$ under the isomorphism $\alpha^{-1}$ is the left $q$-bracketing of the standard word $12 \ldots n$, that is,

$$
\alpha^{-1}\left(\Theta_{n}(q)\right)=\left[\left[\ldots\left[[1,2]_{q}, 3\right]_{q}, \ldots\right]_{q}, n\right]_{q}
$$

where $[R, S]_{q}=R S-q S R$ (see [6]). As an application of the $q$-Newton formulas, let us give the following $q$-analog of Dynkin's theorem, describing the left $q$-bracketing of a homogeneous Lie polynomial:

Proposition 5.6 For all $n \geq 1$,

$$
\Theta_{n}(q) * \Psi_{n}=[n]_{q} \Psi_{n} .
$$

Proof - See Example 3.6. Alternatively, using Proposition 5.4, one can write

$\vartheta(t) * \Psi_{n}=\mu\left(\left(\lambda(A ;-q t) \otimes \sigma_{q}^{\prime}(A ; t)\right) * \Delta\left(\Psi_{n}\right)\right)=\mu\left(\left(\lambda(A ;-q t) \otimes \sigma_{q}^{\prime}(A ; t)\right) *\left(1 \otimes \Psi_{n}+\Psi_{n} \otimes 1\right)\right)$, from which it follows that

$$
\vartheta(t) * \Psi_{n}=(\lambda(A ;-q t) * 1)\left(\sigma_{q}^{\prime}(A ; t) * \Psi_{n}\right)=[n]_{q} \Psi_{n} t^{n-1} .
$$

Example 5.7 Let $P=[a, b]=a b-b a$. Then,

$$
[a, b]_{q}-[b, a]_{q}=a b-q b a-b a+q a b=(1+q)[a, b] .
$$

For $q \neq 1, \Theta_{n}(q)$ does not correspond to an idempotent of the descent algebra. Nevertheless, one can compute its square for the internal product. This will follow from a more general result:

Proposition 5.8 For all $n \geq 1$,

$$
\Theta_{n}(p) * \Theta_{n}(q)=[n]_{p} \Theta_{n}(q)+(q-1) \sum_{i=1}^{n-1} p^{i} q^{i-1}[n-i]_{p} \Theta_{i}(1 / q) \Theta_{n-i}(q) .
$$


Proof - Using again Proposition 5.4, one can write

$$
\Theta_{n}(p) * \Theta_{n}(q)=\mu\left(\left(\lambda(A ;-p t) \otimes \sigma_{p}^{\prime}(A ; t)\right) * \Delta\left(\Theta_{n}(q)\right)\right) .
$$

It follows then from (69) that the coefficient of $t^{n}$ in this last expression (which is the only one different from 0 ) is equal to

$$
[n]_{p} \Theta_{n}(q)+(1-q) \sum_{i=1}^{n-1}(-p)^{i}[n-i]_{p}\left(\Lambda_{i} * \Theta_{i}(q)\right) \Theta_{n-i}(q),
$$

and it it suffices now to observe that

$$
\Lambda_{i} * \Theta_{i}(q)=\omega\left(\Theta_{i}(q)\right)=(-q)^{i-1} \Theta_{i}(1 / q)
$$

according to (71).

Using the basis $\left(R_{I}\right)$, one can give a more precise result:

Corollary 5.9 For $n \geq 1$,

$$
\Theta_{n}(p) * \Theta_{n}(q)=\sum_{|I|=n} C_{I}(p, q) R_{I}(A),
$$

where $C_{I}(p, q)$ is the polynomial of $\mathbb{Z}[p, q]$ defined by

$$
\left\{\begin{array}{cl}
(-1)^{r}\left(q^{r}[r+1]_{p / q}+p^{r+1} q[n-r-1]_{p q}\right) & \text { if } I=\left(1^{r}, n-r\right) \\
(-1)^{t+r-1} p^{r+s-1} q^{t+s-1}(q-1) & \text { if } I=\left(1^{r}, s, 1^{t}, n-r-s-t\right) \text { with } s \geq 2 \\
0 & \text { if } I \neq\left(1^{r}, s, 1^{t}, u\right)
\end{array} .\right.
$$

Proof - Proposition 5.8 shows that

$$
\begin{gathered}
\Theta_{n}(p) * \Theta_{n}(q)=[n]_{p}\left(\sum_{i=0}^{n-1}(-q)^{i} R_{1^{i}, n-i}\right) \\
+(q-1) \sum_{i=1}^{n-1} p^{i} q^{i-1}[n-i]_{p}\left(\sum_{j=0}^{i-1}(-q)^{-j} R_{1^{j}, i-j}\right)\left(\sum_{k=0}^{n-i-1}(-q)^{k} R_{1^{k}, n-i-k}\right) \\
=[n]_{p}\left(\sum_{i=0}^{n-1}(-q)^{i} R_{1^{i}, n-i}\right) \\
+(q-1) \sum_{i=1}^{n-1} \sum_{j=0}^{i-1} \sum_{k=0}^{n-i-1}(-1)^{k-j} p^{i} q^{i+k-j-1}[n-i]_{p} R_{1^{j}, i-j} R_{1^{k}, n-i-k} \\
=[n]_{p}\left(\sum_{i=0}^{n-1}(-q)^{i} R_{1^{i}, n-i}\right) \\
+(q-1) \sum_{i=1}^{n-1} \sum_{j=0}^{i-1} \sum_{k=1}^{n-i-1}(-1)^{k-j} p^{i} q^{i+k-j-1}[n-i]_{p}\left(R_{1^{j}, i-j, 1^{k}, n-i-k}+R_{1^{j}, i-j+1,1^{k-1}, n-i-k}\right)
\end{gathered}
$$




$$
+(q-1) \sum_{i=1}^{n-1} \sum_{j=0}^{i-1}(-1)^{j} p^{i} q^{i-j-1}[n-i]_{p}\left(R_{1^{j}, i-j, n-i}+R_{1^{j}, n-j}\right) .
$$

It follows from this last expression that

$$
\Theta_{n}(p) * \Theta_{n}(q)=\sum_{|I|=n} C_{I}(p, q) R_{I}(A),
$$

where $C_{I}(p, q)$ are polynomials in $\mathbb{Z}[p, q]$. Note that these computations show that $C_{I}(p, q)=$ 0 when $I$ is not of the form $\left(1^{r}, s, 1^{t}, u\right)$, i.e. when $I$ cannot be obtained as a concatenation of two hooks. It is also easy to see that identity (84) shows that

$$
\begin{gathered}
C_{1^{r}, s, 1^{t}, n-r-s-t}(p, q)=(-1)^{t+r} p^{r+s} q^{t+s-1}[n-r-s]_{p}(q-1) \\
+(-1)^{t+r-1} p^{r+s-1} q^{t+s-1}[n-r-s+1]_{p}(q-1) \\
=(-1)^{t+r} p^{r+s-1} q^{t+s-1}(q-1)\left(p[n-r-s]_{p}-[n-r-s+1]_{p}\right) \\
=(-1)^{t+r-1} p^{r+s-1} q^{t+s-1}(q-1)
\end{gathered}
$$

when $s \geq 2$. Using again relation (84), one can also write

$$
\begin{gathered}
C_{1^{r}, n-r}(p, q)=[n]_{p}(-q)^{r}+(q-1)(-1)^{r+1} \sum_{i=1}^{r-1} p^{i} q^{r-i}[n-i]_{p} \\
+(q-1)(-1)^{r+1} p^{r}[n-r]_{p}+(q-1)(-1)^{r} \sum_{i=r+1}^{n-1} p^{i} q^{i-r-1}[n-i]_{p} .
\end{gathered}
$$

Moreover,

$$
\begin{gathered}
(q-1)(-1)^{r+1} \sum_{i=1}^{r} p^{i} q^{r-i}[n-i]_{p}=(-1)^{r+1} \sum_{i=1}^{r-1} p^{i} q^{r-i}\left(p[n-i-1]_{p}-[n-i]_{p}\right) \\
+(-1)^{r+1} q^{r} p[n-1]_{p}+(-1)^{r} p^{r}[n-r]_{p} \\
=(-1)^{r} \sum_{i=1}^{r-1} p^{i} q^{r-i}+(-1)^{r+1} q^{r} p[n-1]_{p}+(-1)^{r} p^{r}[n-r]_{p} .
\end{gathered}
$$

Using (85), this shows that

$$
\begin{gathered}
C_{1^{r}, n-r}(p, q)=(-1)^{r} q^{r}\left([n]_{p}-p[n-1]_{p}\right)+\sum_{i=1}^{r-1}(-1)^{r} p^{i} q^{r-i} \\
+(-1)^{r} p^{r}[n-r]_{p}+(q-1)(-1)^{r} \sum_{i=r+1}^{n-1} p^{i} q^{i-r-1}[n-i]_{p} \\
=\sum_{i=0}^{r-1}(-1)^{r} p^{i} q^{r-i}+(-1)^{r} p^{r}[n-r]_{p}+(q-1)(-1)^{r} \sum_{i=r+1}^{n-1} p^{i} q^{i-r-1}[n-i]_{p} .
\end{gathered}
$$

On the other hand,

$$
(q-1)(-1)^{r} \sum_{i=r+1}^{n-1} p^{i} q^{i-r-1}[n-i]_{p}=(-1)^{r} \sum_{i=r+1}^{n-2} p^{i} q^{-r+i}\left([n-i]_{p}-p[n-i-1]_{p}\right)
$$




$$
\begin{aligned}
& +(-1)^{r+1} p^{r+1}[n-r-1]_{p}+(-1)^{r} p^{n-1} q^{n-r-1} \\
= & (-1)^{r} \sum_{i=r+1}^{n-1} p^{i} q^{-r+i}+(-1)^{r+1} p^{r+1}[n-r-1]_{p} .
\end{aligned}
$$

It follows from this identity that

$$
\begin{gathered}
C_{1^{r}, n-r}(p, q)=(-1)^{r} \sum_{i=0}^{r-1} p^{i} q^{r-i}+(-1)^{r} p^{r}[n-r]_{p}+(-1)^{r+1} p^{r+1}[n-r-1]_{p} \\
+(-1)^{r} \sum_{i=r+1}^{n-1} p^{i} q^{-r+i}=(-1)^{r} \sum_{i=0}^{r} p^{i} q^{r-i}+(-1)^{r} \sum_{i=r+1}^{n-1} p^{i} q^{-r+i} \\
=(-1)^{r}\left(q^{r}[r+1]_{p / q}+p^{r+1} q[n-r-1]_{p q}\right)
\end{gathered}
$$

which concludes the proof.

Note 5.10 According to Lemma 4.19, we have

$$
\Theta_{n}(p) * \Theta_{n}(q)=\frac{S_{n}(((1-p) \hat{\times}(1-q)) A)}{(1-p)(1-q)}
$$

for $n \geq 1$. It follows that Corollary 5.9 gives the expansion of $S_{n}(((1-p) \hat{\times}(1-q)) A)$ on ribbon Schur functions. In particular, the Cauchy formula shows that

$$
C_{I}(p, q)=\frac{F_{I}((1-p) \hat{\times}(1-q))}{(1-p)(1-q)}
$$

for every composition $I$ of $n$.

Another useful property of the $q$-bracketing is given by the following $q$-analog of Lemma 3.7 , which is proved in the same way.

Lemma 5.11 Let $\left(F_{n}\right)$ be a sequence of Lie idempotents, with $F_{n} \in \mathbf{S y m}_{n}$ for each $n$. Then,

$$
\Theta_{n}(q) * F^{I}=\left[i_{1}\right]_{q}\left[\left[\ldots\left[F_{i_{1}}, F_{i_{2}}\right]_{q^{i_{2}}}, \ldots\right]_{q^{i_{r-1}}}, F_{i_{r}}\right]_{q^{i_{r}}} .
$$

Proof - Using the same method as in the proof of Lemma 3.7, one sees that

$$
\Theta_{n}(q) * F^{I}=\left[\Theta_{|J|}(q) * F^{J}, F_{i_{r}}\right]_{q_{r}}+\left[i_{r}\right]_{q} q^{n} \mu\left[(\tilde{\omega} \otimes 1) \Delta\left(F^{J}\right)\right] F_{i_{r}}
$$

where $J=\left(i_{1}, \ldots, i_{r-1}\right)$. The antipodal property of $\tilde{\omega}$ implies that

$$
\Theta_{n}(q) * F^{I}=\left[\Theta_{|J|}(q) * F^{J}, F_{i_{r}}\right]_{q^{i_{r}}}
$$

from which the lemma follows by induction.

Note 5.12 It follows from the lemma that one has

$$
S_{n}((1-q) A) * F^{I}=\left(1-q^{i_{1}}\right)\left[\left[\left[F_{i_{1}}, F_{i_{2}}\right]_{q^{i_{2}}}, \ldots,\right]_{q^{i_{r}-1}}, F_{i_{r}}\right]_{q^{i_{r}}},
$$

under the same hypotheses. 


\subsection{Diagonalization of the left $q$-bracketing}

We have seen in Proposition 5.6 that the Lie polynomials (in the free algebra) are eigenvectors of the standard left $q$-bracketing $a_{1} a_{2} \cdots a_{n} \longmapsto\left[\ldots\left[a_{1}, a_{2}\right]_{q}, \ldots a_{n}\right]_{q}$. It turns out that this operator is semisimple, with $p(n)$ distinct eigenvalues, and that its spectral projectors provide another example of a complete family of orthogonal idempotents of the descent algebra, constructed from a sequence of Lie idempotents by the procedure of Section 3.4.

Instead of $\Theta_{n}(q)$, it will be more convenient to deal with $S_{n}((1-q) A)$ considered as left or right operator for the internal product, i.e. with the operators

$$
F \longrightarrow S_{n}((1-q) A) * F \quad \text { or } \quad F \longrightarrow F * S_{n}((1-q) A)
$$

where $F \in \mathbf{S y m}_{n}$. The eigenvalues of both operators are known from Proposition 3.12:

Lemma 5.13 The eigenvalues of $S_{n}((1-q) A)$ (considered as a left or right operator for the internal product) are the polynomials

$$
\psi^{\lambda}(1-q)=\left(1-q^{\lambda_{1}}\right)\left(1-q^{\lambda_{2}}\right) \ldots\left(1-q^{\lambda_{r}}\right)
$$

where $\lambda=\left(\lambda_{1}, \lambda_{2}, \ldots, \lambda_{r}\right)$ is any partition of $n$.

The problem is now to describe the eigenspaces. This will be done in terms of the corresponding spectral projectors.

Theorem 5.14 There exists a unique family $\pi(q)=\left(\pi_{n}(q)\right)_{n \geq 1}$ ) of Lie idempotents (with $\pi_{n}(q) \in \mathbf{S y m}_{n}$ for all $n$ ) characterized by the property

$$
\pi_{n}(q)((1-q) A)=\left(1-q^{n}\right) \pi_{n}(q)(A) .
$$

Let now $E_{\lambda}(\pi(q))$ be the orthogonal idempotents associated with the family $\pi(q)$ by the process described in Section 3.4. Then, regarding $S_{n}((1-q) A)$ as a left or right operator for the internal product,

1) The element $E_{\lambda}(\pi(q))$ is a projector on the eigenspace of $S_{n}((1-q) A)$ associated with the eigenvalue $\psi^{\lambda}(1-q)$ for every partition $\lambda$ of $n$.

2) $S_{n}((1-q) A)$ is diagonalizable.

Proof - We shall first prove the following lemma.

Lemma 5.15 Suppose that there exist Lie idempotents $\left(\pi_{k}(q)\right)_{k=1, n}$ satisfying to property (91). Then $S_{n}((1-q) A)$ is diagonalizable.

Proof - Consider the orthogonal idempotents $E_{\lambda}(\pi)$ associated with the family $\pi(q)=$ $\left(\pi_{k}(q)\right)_{k=1, n}$ by the method of Section 3.4 for every partition $\lambda$ of $k \leq n$. By definition of these idempotents,

$$
S_{n}(A)=\sum_{\lambda \vdash n} E_{\lambda}(\pi(q))(A)
$$


Changing $A$ into $(1-q) A$ and using property (91), we deduce from this identity that

$$
S_{n}((1-q) A)=\sum_{\lambda \vdash n} E_{\lambda}(\pi(q))((1-q) A)=\sum_{\lambda \vdash n} \psi^{\lambda}(1-q) E_{\lambda}(\pi(q))(A) .
$$

On the other hand, one can write

$$
S_{n}((1-q) A)=S_{n}((1-q) A) * S_{n}(A)=\sum_{\lambda \vdash n} S_{n}((1-q) A) * E_{\lambda}(\pi(q)) .
$$

Finally, it follows from the multihomogeneity of $E_{\lambda}(\pi(q))$ and from (89) that

$$
S_{n}((1-q) A) * E_{\lambda}(\pi(q)) \subset \mathbf{S y m}_{\lambda}(\pi(q))
$$

where $\mathbf{S y m}_{\lambda}(\pi(q))$ denotes the subspace of $\mathbf{S y m}_{n}$ generated by the elements $\pi^{I}(q)$ where $I$ is a composition of $n$ with $\lambda$ as associated partition. Taking into account the fact that

$$
\operatorname{Sym}_{n}=\bigoplus_{\lambda \vdash n} \operatorname{Sym}_{\lambda}(\pi(q))
$$

and relations (92) and (93), we see that

$$
S_{n}((1-q) A) * E_{\lambda}(\pi(q))=\psi^{\lambda}(1-q) E_{\lambda}(\pi(q)) .
$$

Also, using property (91) and Theorem 4.17, it is immediate to get

$$
E_{\lambda}(\pi(q)) * S_{n}((1-q) A)=\psi^{\lambda}(1-q) E_{\lambda}(\pi(q)) .
$$

It follows now from (94) and (95) that $E_{\lambda}(\pi(q))$ may be considered as a (left or right) projector onto the eigenspace associated with $\psi^{\lambda}(1-q)$ for the (left or right) operator $S_{n}((1-q) A)$. The fact that the $E_{\lambda}(\pi(q))$ form a complete set of mutually orthogonal idempotents implies then immediately that $S_{n}((1-q) A)$ is diagonalizable and that the image of $E_{\lambda}(\pi(q))$ is the required eigenspace.

According to Lemma 5.15, it will be sufficient to establish the existence of the family $\pi(q)$ in order to conclude the proof of the theorem. We shall therefore suppose that the $\pi_{k}(q)$ are constructed for $k \leq n-1$. This implies in particular that all operators $S_{k}((1-q) A)$ are diagonalizable for every $k<n$. Let us now consider the polynomial

$$
P(x)=\prod_{\lambda \neq(n)}\left(x-\psi^{\lambda}(1-q)\right)=\sum_{i} p_{i}(q) x^{i} .
$$

We can then give the following lemma. For $F \in \mathbf{S y m}$, let $F^{* k}$ denote the $k$-th power $F * F * \cdots * F$ ( $k$ times), and for a polynomial $P \in K[x]$, let $P^{*}$ be the function on Sym obtained by evaluating $P$ with the internal product.

Lemma 5.16 The noncommutative symmetric function

$$
P_{n}=P^{*}\left(S_{n}((1-q) A)\right)=\sum_{i} p_{i}(q) S_{n}((1-q) A)^{* i}
$$

is a Lie quasi-idempotent of $\mathbf{S y m}_{n}$. 
Proof - We have to check that $P_{n}$ is primitive for $\Delta$. Using the fact that $\Delta$ is a morphism for the internal product, we can write

$$
\begin{gathered}
\Delta\left(P_{n}\right)=\sum_{i} p_{i}(q) \Delta\left(S_{n}((1-q) A)\right)^{* i}=\sum_{i} p_{i}(q)\left(\sum_{k=0}^{n} S_{k}((1-q) A) \otimes S_{n-k}((1-q) A)\right)^{* i} \\
=\sum_{i} p_{i}(q)\left(\sum_{k=0}^{n} S_{k}((1-q) A)^{* i} \otimes S_{n-k}((1-q) A)^{* i}\right) \\
=P_{n} \otimes 1+1 \otimes P_{n}+\sum_{k=1}^{n-1} P^{*}\left(S_{k}((1-q) A) \otimes S_{n-k}((1-q) A)\right) .
\end{gathered}
$$

Using now our induction hypothesis, we get that $S_{k}((1-q) A) \otimes S_{n-k}((1-q) A)$ is diagonalizable for every $k \in[1, n-1]$. Moreover, the eigenvalues of this operator are the $\psi^{\mu}(1-q) \psi^{\nu}(1-q)$, where $\mu$ and $\nu$ are respectively partitions of $k$ and $n-k$. Since these values are all roots of the polynomial $P(x)$, the diagonalizability of the operator $S_{k}((1-q) A) \otimes S_{n-k}\left(S_{n-k}((1-q) A)\right.$ shows that

$$
P^{*}\left(S_{k}((1-q) A) \otimes S_{n-k}((1-q) A=0\right.
$$

for every $k \in[1, n-1]$. It follows that $P_{n}$ is primitive for $\Delta$ as required.

Define now $\pi_{n}(q)$ by

$$
\pi_{n}(q)=\frac{P\left(S_{n}((1-q) A)\right.}{P\left(1-q^{n}\right)} .
$$

The last lemma shows that $\pi_{n}(q)$ is a Lie quasi-idempotent. We shall now show that this Lie quasi-idempotent is in fact a Lie idempotent satisfying to (91). Note first that $\pi_{n}(q)$, being a polynomial in $S_{n}((1-q) A)$, commutes with this element for the internal product. Hence,

$$
S_{n}((1-q) A) * \pi_{n}(q)=\pi_{n}(q) * S_{n}((1-q) A)=\pi_{n}(q)((1-q) A) .
$$

On the other hand, since $\pi_{n}(q)$ is a Lie idempotent, Proposition 5.6 implies that

$$
S_{n}((1-q) A) * \pi_{n}(q)=\left(1-q^{n}\right) \pi_{n}(q) .
$$

The conjunction of the last two identities shows then that $\pi_{n}(q)$ satisfies to property (91). It remains to prove that $\left.\pi_{(} q\right)$ is actually a Lie idempotent, i.e. that

$$
\pi_{n}(q) \in \frac{\Psi_{n}}{n}+[L(\Psi), L(\Psi)]
$$

Let us therefore calculate the multiplicity of $\pi_{n}(q)$ on $\Psi_{n}$. Recall first that

$$
S_{n}((1-q) A)=\frac{1-q^{n}}{n} \Psi_{n}+[L(\Psi), L(\Psi)] .
$$

Then, it follows from Example 3.8 that

$$
S_{n}((1-q) A)^{* i}=\left(\frac{1-q^{n}}{n}\right)^{i} \Psi_{n}^{* i}+[L(\Psi), L(\Psi)]=\frac{\left(1-q^{n}\right)^{i}}{n} \Psi_{n}+[L(\Psi), L(\Psi)]
$$


for every $i \geq 0$. Hence,

$$
P^{*}\left(S_{n}((1-q) A)\right)=P\left(1-q^{n}\right) \frac{\Psi_{n}}{n}+[L(\Psi), L(\Psi)],
$$

which shows that $\pi_{n}(q)$ is a Lie idempotent. To finish the proof of the theorem, it suffices to prove the unicity of the family $\pi(q)$. Thus, suppose that $F_{n}$ is a Lie idempotent such that $F_{n} * S_{n}((1-q) A)=\left(1-q^{n}\right) F_{n}$. As $\pi_{1}, \ldots, \pi_{n}$ are Lie idempotents, one can write

$$
F_{n}=\pi_{n}+\sum_{\ell(I) \geq 2} c_{I} \pi^{I}
$$

so that, multiplying on the right by $S_{n}((1-q) A)$, we find

$$
F_{n}=\pi_{n}+\sum_{\ell(I) \geq 2} \frac{\psi^{I}(1-q)}{1-q^{n}} c_{I} \pi^{I}
$$

whence $c_{I} \equiv 0$ by linear independence of the $\pi^{I}$.

Example 5.17 Here are the first idempotents $\pi_{n}(q)$ for $n \leq 5$ :

$$
\begin{gathered}
\pi_{1}(q)=\Psi_{1}, \quad \pi_{2}(q)=\frac{\Psi_{2}}{2}, \quad \pi_{3}(q)=\frac{\Psi_{3}}{3}+\frac{1}{6} \frac{1-q}{(1+2 q)}\left[\Psi_{2}, \Psi_{1}\right], \\
\pi_{4}(q)=\frac{\Psi_{4}}{4}+\frac{1}{12} \frac{(1-q)(2 q+1)}{\left(1+q+2 q^{2}\right)}\left[\Psi_{3}, \Psi_{1}\right]+\frac{1}{24} \frac{(1-q)^{2}}{\left(1+q+2 q^{2}\right)}\left[\left[\Psi_{2}, \Psi_{1}\right], \Psi_{1}\right], \\
\pi_{5}(q)=\frac{\Psi_{5}}{5}+\frac{1}{20} \frac{(1-q)\left(3 q^{2}+2 q+1\right)}{\left(2 q^{3}+q^{2}+q+1\right)}\left[\Psi_{4}, \Psi_{1}\right]+\frac{1}{30} \frac{(1-q)(q+2)}{\left(2 q^{2}+2 q+1\right)}\left[\Psi_{3}, \Psi_{2}\right] \\
+\frac{1}{60} \frac{(1-q)^{2}\left(4 q^{3}+7 q^{2}+7 q+2\right)}{\left(2 q^{2}+q+2\right)\left(2 q^{3}+q^{2}+q+1\right)}\left[\left[\Psi_{3}, \Psi_{1}\right], \Psi_{1}\right] \\
\quad-\frac{1}{120} \frac{(1-q)^{2}\left(4 q^{2}+9 q+7\right)}{\left(q^{2}+3 q+1\right)\left(2 q^{2}+1+2 q\right)}\left[\left[\Psi_{1}, \Psi_{2}\right], \Psi_{2}\right] \\
+\frac{1}{120} \frac{(1-q)^{3}\left(2 q^{5}+2 q^{4}+q^{3}+5 q^{2}+9 q+6\right)}{\left(2 q^{3}-q^{2}+q+3\right)\left(2 q^{2}+q+2\right)\left(2 q^{3}+q^{2}+q+1\right)}\left[\left[\left[\Psi_{2}, \Psi_{1}\right], \Psi_{1}\right], \Psi_{1}\right] .
\end{gathered}
$$

Example 5.18 We also give here the first orthogonal projectors on the different eigenspaces associated with the operators $S_{n}((1-q) A)$.

$$
\begin{gathered}
E_{1}=\Psi_{1}, E_{2}=\frac{\Psi_{2}}{2}, E_{11}=\frac{\Psi^{11}}{2}, \\
E_{3}=\frac{\pi_{3}(q)}{3}, E_{21}=\frac{1}{2(1+2 q)}\left(q \Psi^{21}+(1+q) \Psi^{12}\right), E_{111}=\frac{\Psi^{111}}{6}, \\
E_{4}=\frac{\pi_{4}(q)}{4}, E_{31}=\frac{1}{1+q+2 q^{2}}\left(q^{2} \pi^{31}(q)+\left(q^{2}+q+1\right) \pi^{13}(q)\right), E_{22}=\frac{\Psi^{22}}{8}, \\
E_{211}=\frac{1}{4(1+2 q)}\left(q \Psi^{211}+2 q \Psi^{121}+(q+2) \Psi^{112}\right), E_{1111}=\frac{\Psi^{1111}}{24} .
\end{gathered}
$$


One can recover from Theorem 5.14 the character of the left ideal $\mathbb{C S}_{n} \eta_{n}(q)$, as computed in [6], or, which amounts to the same, the character $f_{n}(q)$ of the linear group $G L(V)$ of a vector space $V$ in $T^{n}(V) \eta_{n}(q)$.

For $q$ not a root of unity of order $n$ or less, the element $S_{n}(A /(1-q))$ is well defined, and invertible for the internal product, with inverse $S_{n}((1-q) A)$. So in this case, $f_{n}(q)=p_{1}^{n}$.

When $q=\omega$ is a root of unity, $W:=T^{n}(V) \eta_{n}(\omega)$ is the direct sum of the eigenspaces $W_{\lambda}:=T^{n}(V) e_{\lambda}(\omega)$ corresponding to nonzero eigenvalues (here, the $e_{\lambda}$ are the spectral projectors constructed in this section). Since these eigenvalues are $t_{\lambda}=\psi^{\lambda}(1-\omega)$, one obtains, taking into account 3.12 :

Corollary 5.19 [6] The character of $G L(V)$ in the image $W=T^{n}(V) \eta_{n}(\omega)$ of the iterated $q$-bracketing at a root of unity is

$$
f_{n}(\omega)=\sum_{w_{\lambda}(\omega) \neq 0} L_{\lambda}
$$

where $w_{\lambda}(q)=\psi^{\lambda}(1-q) /(1-q)$. If $\omega$ is a primitive $k$-th root of unity, the generating series of the characters is

$$
\sum_{n \geq 0} f_{n}(\omega)=\sigma_{1}\left[\sum_{m \neq 0 \bmod k} \ell_{m}\right]
$$

where $\sigma_{1}=\sum_{n \geq 0} h_{n}, f[g]$ is the plethysm of $g$ by $f$, and $\ell_{n}=(1 / n) \sum_{d \mid n} \mu(d) p_{d}^{n / d}$.

Note 5.20 The arguments used in the proof of Proposition 5.14 can be extended to a more general situation. Indeed, let $X$ be an arbitrary totally ordered alphabet. Our method shows then that the operator $S_{n}(X A)$ is diagonalizable with $\psi^{\lambda}(X)$ as eigenvalues (where $\lambda$ is a partition of $n$ ) and that the corresponding eigenspaces can still be constructed by the method of Section 3.4 based on the unique family of Lie idempotents $\pi_{X, n}$ satisfying to the property

$$
\pi_{X, n}(X A)=\psi_{n}(X) \pi_{X, n}(A)
$$

Example 5.21 We here give the first values of $\pi_{X, n}$. Their coefficients are rational quasisymmetric functions of $X$.

$$
\begin{gathered}
\pi_{X, 1}=\Psi_{1}, \quad \pi_{X, 2}=\frac{\Psi_{2}}{2} \\
\pi_{X, 3}=\frac{\Psi_{3}}{3}+\frac{\frac{1}{6} M_{12}-\frac{1}{3} M_{21}}{M_{12}+M_{21}}\left[\Psi_{2}, \Psi_{1}\right] .
\end{gathered}
$$

For $n=1$ and $n=2$, the associated orthogonal idempotents do not differ from the $q$-case. For $n=3$, we have

$$
E_{3}=\pi_{X, 3}, E_{21}=\frac{M_{21} \pi_{X}^{21}+M_{12} \pi_{X}^{12}}{M_{12}+M_{21}}, E_{111}=\frac{\Psi^{111}}{6}
$$




\subsection{Specializations of the family $\pi_{n}(q)$}

The idempotents $\pi_{n}(q)$ constructed in the preceding section have interesting specializations. The most easily identified is for $q=1$.

Proposition 5.22 For every $n \geq 1$, one has

$$
\pi_{n}(1)=\frac{\Psi_{n}}{n}
$$

Proof - Using again the notations of the proof of Proposition 5.14, we can write

$$
\pi_{n}(q)=\frac{P\left(S_{n}((1-q) A)\right)}{P\left(1-q^{n}\right)}=\frac{P\left(S_{n}((1-q) A)\right)}{(1-q)^{p(n)-1}} \frac{(1-q)^{p(n)-1}}{P\left(1-q^{n}\right)} .
$$

But one clearly has

$$
P(x)=\prod_{\lambda \neq(n)}\left(x-\psi^{\lambda}(1-q)\right) \longrightarrow_{q \rightarrow 1} x^{p(n)-1},
$$

where $p(n)$ denotes the number of partitions of $n$. Observe now that

$$
\frac{P\left(S_{n}((1-q) A)\right)}{(1-q)^{p(n)-1}} \simeq{ }_{q \rightarrow 1} \frac{S_{n}((1-q) A)}{(1-q)^{*(p(n)-1)}} \longrightarrow_{q \rightarrow 1} \Psi_{n}^{*(p(n)-1)}=n^{p(n)-2} \Psi_{n} .
$$

On the other hand,

$$
\frac{(1-q)^{p(n)-1}}{P\left(1-q^{n}\right)}=\frac{1}{\prod_{\lambda \neq(n)}\left(\frac{1-q^{n}}{1-q}-\frac{\psi^{\lambda}(1-q)}{1-q}\right)} \rightarrow_{q \rightarrow 1} \frac{1}{n^{p(n)-1}} .
$$

Corollary 5.23 Let $F_{n}$ be some homogenous Lie idempotent of order $n$. Then one has

$$
\lim _{q \rightarrow 1} \frac{F_{n}((1-q) A)}{1-q^{n}}=\frac{\Psi_{n}}{n} .
$$

Proof - Decomposing $F_{n}$ in the basis $\left(\pi^{I}(q)\right)$, we have

$$
F_{n}=\pi_{n}(q)+\sum_{\ell(I) \geq 2} a_{I}(q) \pi^{I}(q)
$$

Using the basic property of the idempotents $\pi_{n}(q)$, we get

$$
\frac{F_{n}((1-q) A)}{1-q^{n}}=\pi_{n}(q)+\sum_{\ell(I) \geq 2} a_{I}(q) \frac{\psi^{I}(1-q)}{1-q^{n}} \pi^{I}(q)
$$

where $\psi^{I}(1-q)=\left(1-q^{i_{1}}\right) \ldots\left(1-q^{i_{r}}\right)$. Proposition 5.22 shows that $\pi_{n}(q)$ converges to $\frac{\Psi_{n}}{n}$ when $q \rightarrow 1$. It follows that $a_{I}(q)$ converges when $q \rightarrow 1$ (up to some normalization) 
to the coefficient $a_{I}$ corresponding to the decomposition of $F_{n}$ on the basis $\Psi^{I}$. Since one also clearly has

$$
\lim _{q \rightarrow 1} \frac{\psi^{I}(1-q)}{1-q^{n}}=0
$$

for every composition $I$ of length greater or equal to 2 , we obtain that

$$
\lim _{q \rightarrow 1} \frac{F_{n}((1-q) A)}{1-q^{n}}=\frac{\Psi_{n}}{n}
$$

as desired.

The following result shows that the specialization of the idempotent $\pi_{n}(q)$ defined by Proposition 5.14 at a primitive $n$-th root of unity is equal to Klyachko's idempotent.

Proposition 5.24 Let $\zeta$ be a primitive $n$-th root of unity. Then one has

$$
\pi_{n}(\zeta)=\kappa_{n}(\zeta)
$$

Proof - Let $Q(x)$ be the minimal polynomial of $S_{n}((1-q) A)$. Using again the notations of the proof of Proposition 5.14, we can write

$$
Q(X)=P(X)\left(X-\left(1-q^{n}\right)\right)
$$

On the other hand, let us set

$$
Q(X)=\sum_{i} q_{i}(q) X^{i}
$$

It follows that one has

$$
\left(\frac{Q(X)-q_{0}(q)}{X}\right) X=-q_{0}(q)+Q(X) .
$$

Substituting now $X$ to $S_{n}((1-q) A)$ in this expression, we find that the inverse of $S_{n}((1-$ $q) A$ ) for the internal product is equal to

$$
S_{n}\left(\frac{A}{1-q}\right)=\left.\left(\frac{q_{0}(q)-Q(X)}{q_{0}(q) X}\right)\right|_{X=S_{n}((1-q) A)} .
$$

Observe now that

$$
q_{0}(q)=\left(q^{n}-1\right) q_{0}^{\prime}(q)
$$

where

$$
q_{0}^{\prime}(q)=\prod_{\lambda \neq(n)} \psi^{\lambda}(q-1)
$$

It follows that

$$
\begin{gathered}
K_{n}(q)=(q)_{n} S_{n}\left(\frac{A}{1-q}\right)=\left.(q)_{n}\left(\frac{q_{0}(q)-P(X)\left(X-\left(1-q^{n}\right)\right)}{\left(q^{n}-1\right) q_{0}^{\prime}(q) X}\right)\right|_{X=S_{n}((1-q) A)} \\
=\left.(q)_{n-1} \frac{P(X)}{q_{0}^{\prime}(q)}\right|_{X=S_{n}((1-q) A)}+\left.(q)_{n}\left(\frac{\left.q_{0}^{\prime}(q)-P(X)\right)}{q_{0}^{\prime}(q) X}\right)\right|_{X=S_{n}((1-q) A)} .
\end{gathered}
$$


Let now $\zeta$ be a primitive $n$-th root of unity. Using the fact that $(\zeta)_{n}=0$ and $(\zeta)_{n-1}=n$, we get from this last relation

$$
K_{n}(\zeta)=\left.n \frac{P\left(S_{n}((1-q) A)\right)}{q_{0}^{\prime}(\zeta)}\right|_{q=\zeta}=n \pi_{n}(\zeta),
$$

since we clearly have

$$
\left.P\left(1-q^{n}\right)\right|_{q=\zeta}=\left.P(0)\right|_{q=\zeta}=q_{0}^{\prime}(\zeta)
$$

Hence,

$$
\pi_{n}(\zeta)=\frac{K_{n}(\zeta)}{n}=\kappa_{n}(\zeta)
$$

as required.

Corollary 5.25 Let $F_{n}$ be some homogenous Lie idempotent of order $n$ and let $\zeta$ be some primitive $n$-th root of unity. Then one has

$$
\lim _{q \rightarrow \zeta}\left(1-q^{n}\right) F_{n}\left(\frac{A}{1-q}\right)=\kappa_{n}(\zeta) .
$$

Proof - The technique is essentially the same as for proving Corollary 5.23. Decomposing $F_{n}$ in the basis $\left(\pi^{I}(q)\right)$, we have

$$
F_{n}=\pi_{n}(q)+\sum_{\ell(I) \geq 2} a_{I}(q) \pi^{I}(q)
$$

Using the basic property of the idempotents $\pi_{n}(q)$, we get

$$
\left(1-q^{n}\right) F_{n}\left(\frac{A}{1-q}\right)=\pi_{n}(q)+\sum_{\ell(I) \geq 2} a_{I}(q) \frac{\left(1-q^{n}\right)}{\psi^{I}(1-q)} \pi^{I}(q),
$$

where $\psi^{I}(1-q)=\left(1-q^{i_{1}}\right) \ldots\left(1-q^{i_{r}}\right)$. Proposition 5.24 shows that $\pi_{n}(q)$ converges to $\kappa_{n}(\zeta)$ when $q \rightarrow \zeta$. It follows that $a_{I}(q)$ converges when $q \rightarrow \zeta$ (up to some normalization) to the coefficient $a_{I}$ corresponding to the decomposition of $F_{n}$ on the basis $\kappa^{I}$. Since one also has

$$
\lim _{q \rightarrow \zeta} \frac{1-q^{n}}{\psi^{I}(1-q)}=0
$$

for any composition $I$ of length greater or equal to 2 , we obtain that

$$
\lim _{q \rightarrow \zeta}\left(1-q^{n}\right) F_{n}\left(\frac{A}{1-q}\right)=\kappa_{n}(\zeta)
$$

as desired.

To describe the next specialization, we need to introduce a new family of noncommutative power sums.

Definition 5.26 The noncommutative power sum symmetric functions of the third kind $Z_{n}$ are defined by the relation

$$
\sigma(A ; t)=\exp \left(Z_{1} t\right) \exp \left(\frac{Z_{2}}{2} t^{2}\right) \ldots \exp \left(\frac{Z_{n}}{n} t^{n}\right) \ldots
$$


The Fer-Zassenhauss formula ( $c f$. [39]) shows that every $Z_{n}$ is a Lie element. It is also clear that $Z_{n}$ reduces to $\psi_{n}$ in the commutative case.

Example 5.27 The first values of $Z_{n}$ are

$$
\begin{gathered}
Z_{1}=\Psi_{1}, Z_{2}=\Psi_{2}, Z_{3}=\Psi_{3}+\frac{1}{2}\left[\Psi_{2}, \Psi_{1}\right], \\
Z_{4}=\Psi_{4}+\frac{1}{3}\left[\Psi_{3}, \Psi_{1}\right]+\frac{1}{6}\left[\left[\Psi_{2}, \Psi_{1}\right], \Psi_{1}\right], \\
Z_{5}=\Psi_{5}+\frac{1}{4}\left[\Psi_{4}, \Psi_{1}\right]+\frac{1}{3}\left[\Psi_{3}, \Psi_{2}\right]+\frac{1}{12}\left[\left[\Psi_{3}, \Psi_{1}\right], \Psi_{1}\right] \\
-\frac{7}{24}\left[\Psi_{2},\left[\Psi_{2}, \Psi_{1}\right]\right]+\frac{1}{24}\left[\left[\left[\Psi_{2}, \Psi_{1}\right], \Psi_{1}\right], \Psi_{1}\right] .
\end{gathered}
$$

Note 5.28 The explicit formula of Goldberg (see [33]) for the Hausdorff series gives the decomposition of $\Phi_{n}$ on the basis $Z^{I}$.

The specialization of $\pi_{n}(q)$ for $q=0$ seems to be the following:

Conjecture 5.29 For every $n \geq 1$, one has

$$
\pi_{n}(0)=\frac{Z_{n}}{n} .
$$

\subsection{Other expansions}

\subsection{1 $S_{n}((1-q) A)$ and $\Lambda_{n}((1-q) A)$}

Using the quasi-determinantal expressions given by Proposition 5.5, or the corresponding $q$-integral formulas, we obtain :

Proposition 5.30 One has

$$
\begin{gathered}
S_{n}((1-q) A)=\sum_{|I|=n}(-1)^{\ell(I)-1} q^{n-i_{r}}\left(1-q^{i_{r}}\right) S^{I}(A), \\
S_{n}(A)=\sum_{|I|=n} \frac{q^{\operatorname{maj}(I)}}{\left(1-q^{i_{1}}\right)\left(1-q^{i_{1}+i_{2}}\right) \ldots\left(1-q^{i_{1}+\ldots+i_{r}}\right)} S^{I}((1-q) A),
\end{gathered}
$$

the above sums being taken over all compositions $I=\left(i_{1}, i_{2}, \ldots, i_{r}\right)$ of $n$.

Example 5.31 For $n=2$ and $n=3$,

$$
\begin{gathered}
M(S((1-q) A), S)_{2}=\left(\begin{array}{ccc}
1-q^{2} & -q(1-q) \\
0 & 1-q
\end{array}\right) \\
M(S((1-q) A), S)_{3}=\left(\begin{array}{cccc}
1-q^{3} & -q^{2}(1-q) & -q\left(1-q^{2}\right) & q^{2}(1-q) \\
0 & 1-q^{2} & 0 & -q(1-q) \\
0 & 0 & 1-q^{2} & -q(1-q) \\
0 & 0 & 0 & 1-q
\end{array}\right) .
\end{gathered}
$$


Corollary 5.32 One has

$$
\Lambda_{n}((1-q) A)=\sum_{|I|=n}(-1)^{n-\ell(I)}\left(1-q^{i_{r}}\right) S^{I}(A)
$$

the sum being taken over all compositions $I=\left(i_{1}, \ldots, i_{r}\right)$ of $n$.

Proof - This follows from (105) and from Proposition 5.3, which shows that $\Lambda_{n}((1-$ q)A) $=(-q)^{n} S_{n}((1-1 / q) A)$.

\subsection{2 $\Psi_{n}((1-q) A)$}

One can also give the expansion of $\Psi_{n}((1-q) A)$ on $S^{I}(A)$ and $R_{I}(A)$.

Proposition 5.33 One has

$$
\Psi_{n}((1-q) A)=\sum_{|I|=n}(-1)^{\ell(I)-1}\left(1-q^{i_{r}}\right) \underline{I}(q) S^{I}(A)
$$

where for a composition $I=\left(i_{1}, \ldots, i_{r}\right)$ of $n$, we denote by $\underline{I}(q)$ the polynomial of $\mathbb{N}[q]$

$$
\underline{I}(q)=i_{r}+i_{r-1} q^{i_{r-1}}+i_{r-2} q^{i_{r-1}+i_{r-2}}+\ldots+i_{1} q^{i_{r-1}+\ldots+i_{1}} .
$$

Proof - According to Corollary 4.19,

$$
\Psi_{n}((1-q) A)=\Psi_{n} * S_{n}((1-q) A)=(1-q) \Theta_{n}(1) * \Theta_{n}(q)
$$

for every $n \geq 1$. Then, Proposition 5.8 shows that

$$
\Psi_{n}((1-q) A)=n S_{n}((1-q) A)+\sum_{i=1}^{n-1}(n-i) q^{i} S_{i}((1-1 / q) A) S_{n-i}((1-q) A)
$$

Let now $I=\left(i_{1}, \ldots, i_{r}\right)$ be a composition of $n$. It follows from (105) and of this last identity that the coefficient of $\Psi_{n}((1-q) A)$ on $S^{I}(A)$ is equal to

$$
(-1)^{\ell(I)-1}\left(1-q^{i_{r}}\right)\left(n q^{n-i_{r}}-\sum_{k=1}^{r-1}\left(i_{k+1}+\ldots+i_{r}\right)\left(1-q^{-i_{k}}\right) q^{i_{k}+\ldots+i_{r-1}}\right) .
$$

Now, a simple computation shows that

$$
\begin{gathered}
n q^{n-i_{r}}-\sum_{k=1}^{r-1}\left(i_{k+1}+\ldots+i_{r}\right)\left(1-q^{-i_{k}}\right) q^{i_{k}+\ldots+i_{r-1}} \\
=\sum_{k=1}^{r-1} q^{i_{k}+\ldots+i_{r-1}}\left(-i_{k+1}-\ldots-i_{r}\right)+\sum_{k=1}^{r} q^{i_{k}+\ldots+i_{r-1}}\left(i_{k}+\ldots+i_{r}\right)=\sum_{k=1}^{r} i_{k} q^{i_{k}+\ldots+i_{r-1}}
\end{gathered}
$$

which is exactly $\underline{I}(q)$. 
Proposition 5.34 One has

$$
\Psi_{n}((1-q) A)=(1-q) \sum_{|I|=n} C_{I}(q) R_{I}(A)
$$

where $C_{I}(q)$ is the polynomial of $\mathbb{Z}[q]$ defined by

$$
\left\{\begin{array}{cl}
(-1)^{r}\left(q^{r}[r+1]_{1 / q}+q[n-r-1]_{q}\right) & \text { if } I=\left(1^{r}, n-r\right) \\
(-1)^{t+r-1} q^{t+s-1}(q-1) & \text { if } I=\left(1^{r}, s, 1^{t}, n-r-s-t\right) \text { with } s \geq 2 \\
0 & \text { if } I \neq\left(1^{r}, s, 1^{t}, u\right)
\end{array} .\right.
$$

Proof - This follows immediately from relation (110) and Corollary 5.9.

Note 5.35 The polynomials $C_{I}(q)$ are given by the following specialization of the quasiribbons :

$$
C_{I}(q)=\left.\frac{F_{I}((1-p) \hat{\times}(1-q))}{(1-p)(1-q)}\right|_{p=1}
$$

One can prove that

$$
\sum_{|I|=n} C_{I}(q)(-x)^{\ell(I)-1}=[n]_{q}[n]_{x}
$$

for $n \geq 1$. Indeed, the (commutative) specialization $A=1-x$, i.e. $\Psi_{n}(1-x)=1-x^{n}$ and $S_{k}(1-x)=1-x$ for $k \geq 1$ gives for ribbons $R_{I}(A)=(1-x)(-x)^{\ell(I)-1}$ so that

$$
\left(1-q^{n}\right)\left(1-x^{n}\right)=\Psi_{n}((1-q)(1-x))=(1-q) \sum_{|I|=n} C_{I}(q)(1-x)(-x)^{\ell(I)-1} .
$$

\subsection{3 $\Phi_{n}((1-q) A)$}

Let $H(a, b)$ denote the Lie series defined by the two variable Baker-Campbell-Hausdorff formula

$$
H(a, b)=\log \left(e^{a} e^{b}\right)
$$

One can then state

Proposition 5.36 One has

$$
\Phi((1-q) A ; t):=\sum_{n \geq 1} \frac{\Phi_{n}((1-q) A)}{n} t^{n}=H(-\Phi(A ;-q t), \Phi(A ; t)) .
$$

Proof - Indeed,

$$
\Phi((1-q) A)=\log (\sigma((1-q) A ; t))=\log \left(\sigma(A ; q t)^{-1} \sigma(A ; t)\right)=\log \left(e^{-\Phi(A ; q t)} e^{\Phi(A ; t)}\right),
$$

from which the conclusion follows. 
Note 5.37 Corollary 5.22 implies that

$$
\lim _{q \longrightarrow 1} \frac{\Phi_{n}((1-q) A)}{1-q^{n}}=\Psi_{n} .
$$

This shows that the expansion of $\Psi_{n}$ on the basis $\Phi^{I}$ is directly connected to the two variable Baker-Campbell-Hausdorff formula. To illustrate this point, let us recall that the expansion of $H$ up to the order 2 is equal to

$$
H(a, b)=a+b+\frac{1}{2}[a, b]+\ldots .
$$

A simple computation based on Proposition 5.36 shows then that

$$
\begin{gathered}
\frac{\Phi_{n}((1-q) A)}{n}=\left(1-q^{n}\right) \frac{\Phi_{n}(A)}{n}+\frac{1}{2} \sum_{i=1}^{n-1} \frac{q^{i}}{i(n-i)}\left[\Phi_{n-i}(A), \Phi_{i}(A)\right]+\ldots \\
=\left(1-q^{n}\right) \frac{\Phi_{n}(A)}{n}+\frac{1}{2} \sum_{i=1}^{[n / 2]} \frac{\left(q^{i}-q^{n-i}\right)}{i(n-i)}\left[\Phi_{n-i}(A), \Phi_{i}(A)\right]+\ldots
\end{gathered}
$$

Using now relation (117), we get the Lie expansion of $\Psi_{n}(A)$ on the basis $\Phi^{I}(A)$

$$
\Psi_{n}(A)=\Phi_{n}(A)+\sum_{i=1}^{[n / 2]} \frac{n-2 i}{2 i(n-i)}\left[\Phi_{n-i}(A), \Phi_{i}(A)\right]+\ldots,
$$

the terms of higher order of $H$ giving the terms of higher order of this Lie expansion.

\subsection{4 $R_{I}((1-q) A)$}

The decomposition of $R_{I}((1-q) A)$ on the basis $\left(R_{J}\right)$, or, which amounts to the same, the internal products $R_{I} * R_{\left(1^{k}, n-k\right)}$, can be deduced from a result of Blessenohl and Laue [5]. To state it, we need the following definitions.

For any two compositions $I, J$ of $n$ we define an integer $b(I, J) \in \mathbb{N} \cup\{-\infty\}$ inductively by $b(\emptyset, \emptyset)=0$ and

$$
\begin{aligned}
& b(k I, 1 J)=\left\{\begin{array}{cc}
b(I, J) & \text { if } k=1 \\
b((k-1) I, J)+1 & \text { if } k \geq 2
\end{array},\right. \\
& b(k I, 2 J)=\left\{\begin{array}{cc}
-\infty & \text { if } k \geq 3 \\
b(I, J) & \text { if } k=2
\end{array} \quad, \quad b(1 k I, 2 J)=\left\{\begin{array}{cc}
-\infty & \text { if } k=1 \\
b(k-1, J)+1 & \text { if } k \geq 2
\end{array},\right.\right. \\
& b(k I, l J)=\left\{\begin{array}{cc}
b(I,(l-1) J)+1 & \text { if } k=1 \text { and } l \geq 3 \\
b((k-1) I,(l-1) J) & \text { if } k \geq 2 \text { and } l \geq 3
\end{array} .\right.
\end{aligned}
$$

These relations just mean that the matrix $B_{n}=(b(I, J))_{I, J \models n}$ (indexed in the usual way) satisfies to $B_{1}=(0)$ and

$$
B_{n}=\left(\begin{array}{cc}
a_{n} & B_{n-1}+J_{n-1} \\
b_{n} & B_{n-1}
\end{array}\right)
$$

where $a_{n}$ and $b_{n}$ are square matrices of order $2^{n-2}$ defined by $a_{1}=(0), b_{1}=(1)$ and the relations

$$
a_{n}=\left(\begin{array}{cc}
a_{n-1} & -\infty \\
b_{n-1} & B_{n-2}
\end{array}\right) \quad, b_{n}=\left(\begin{array}{cc}
a_{n-1}+J_{n-2} & B_{n-2}+J_{n-2} \\
b_{n-1}+J_{n-2} & -\infty
\end{array}\right)
$$


where $J_{m}$ denotes the matrix of order $2^{m-1}$ whith all entries equal to 1 .

A composition $I$ can be written as a concatenation

$$
I=H_{1} \cdot H_{2} \cdots H_{h l(I)}
$$

of hook compositions $H_{i}=\left(1^{k}, l\right)$. The integer $h l(I)$ will be called the hook length of $I$. The subset of $[1, n-1]$ associated with the composition $\left(\ell\left(H_{1}\right), \ell\left(H_{2}\right), \ldots, \ell\left(H_{h l(I)}\right)\right)$ will be denoted by $H P(I)$. For instance, with $I=(1,3,1,4,2)$

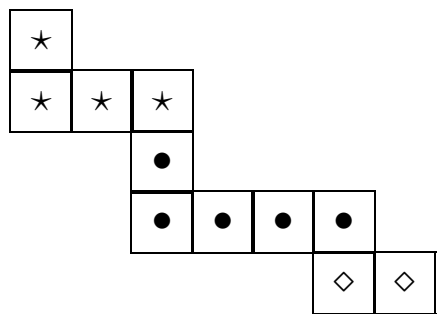

one has $h l(I)=3$ and $H P(I)=\{4,9\}$.

Lemma 5.38 Let $I, J$ be two compositions of $n$ and let $E(I), E(J)$ be the corresponding subsets of $[1, n-1]$. Then,

1) $b(I, J) \neq-\infty$ iff $H P(J) \subset((1+E(I))-E(I)) \cup(E(I)-(1+E(I)))$,

2) For every $I, J$ such that $b(I, J) \neq-\infty$, one has

$$
b(I, J)=|(1+(E(I)-E(J))) \cup(E(J)-E(I))| .
$$

We also need the following lemma.

Lemma 5.39 For every compositions $I, J$ of $n$, one has $h l(J)+b(I, J) \leq n$.

The following result is due to Blessenohl and Laue [5].

Proposition 5.40 Let $I$ be a composition of $n$ and let $0 \leq k<n$. Then,

$$
R_{I} * R_{\left(1^{k}, n-k\right)}=\sum_{J}\left(\begin{array}{c}
h l(J)-1 \\
k-b(I, J)
\end{array}\right) R_{J}
$$

the sum being taken over all $J$ such that $b(I, J) \neq-\infty$.

Set $(-q)^{-\infty}=0$. As a consequence of Proposition 5.40, we obtain a closed expression for the ribbon Schur functions of the alphabet $(1-q) A$ on the usual ribbon functions.

Proposition 5.41 For every composition I of $n$, one has

$$
R_{I}((1-q) A)=\sum_{J}(1-q)^{h l(J)}(-q)^{b(I, J)} R_{J} .
$$


Proof - One can clearly write

$$
R_{I}((1-q) A)=R_{I} * S_{n}((1-q) A)=(1-q) \sum_{k=0}^{n-1}(-q)^{k} R_{I} * R_{1^{k}, n-k} .
$$

Using now Proposition 5.40, one obtains

$$
\begin{gathered}
R_{I}((1-q) A)=(1-q) \sum_{J}\left(\sum_{k=0}^{n-1}\left(\begin{array}{c}
h l(J)-1 \\
k-b(I, J)
\end{array}\right)(-q)^{k}\right) R_{J} \\
=(1-q) \sum_{J}(-q)^{b(I, J)}\left(\sum_{k=0}^{h l(J)-1}\left(\begin{array}{c}
h l(J)-1 \\
k
\end{array}\right)(-q)^{k}\right) R_{J} \\
=\sum_{J}(-q)^{b(I, J)}(1-q)^{h l(J)} R_{J},
\end{gathered}
$$

since one always has $h l(J)+b(I, J) \leq n$ according to Lemma 5.39.

Example 5.42 For $n=2$ and $n=3$, one has

$$
\begin{gathered}
M\left(R_{I}((1-q) A), R_{I}\right)_{2}=\left(\begin{array}{cc}
1-q & -q(1-q) \\
-q(1-q) & 1-q
\end{array}\right) \\
M\left(R_{I}((1-q) A), R_{I}\right)_{3}=\left(\begin{array}{cccc}
1-q & 0 & -q(1-q) & q^{2}(1-q) \\
-q(1-q) & (1-q)^{2} & q^{2}(1-q) & -q(1-q) \\
-q(1-q) & -q(1-q)^{2} & 1-q & -q(1-q) \\
q^{2}(1-q) & 0 & -q(1-q) & 1-q
\end{array}\right) .
\end{gathered}
$$




\section{Symmetric functions of the alphabet $A /(1-q)$}

To define the noncommutative alphabet $A /(1-q)$, one has to choose a total ordering on the commutative alphabet $\left\{1, q, q^{2}, \ldots\right\}$. This is done in such a way that the transformations $A \rightarrow(1-q) A$ and $A \rightarrow A /(1-q)$ be inverse to each other.

\subsection{Complete functions}

Let $X_{q}$ denote the alphabet $X_{q}=\left\{1, q, q^{2}, \ldots, q^{n}, \ldots\right\}$. We totally order it by setting

$$
\ldots<q^{n}<\ldots<q^{2}<q<1 \text {. }
$$

According to Section 4, we can define the complete symmetric functions of the alphabet $X_{q} A$ (that we shall rather denote here by $\frac{A}{1-q}$ ) by its generating series as follows.

Definition 6.1 The generating series of the complete symmetric functions $S_{n}\left(\frac{A}{1-q}\right)$ of the alphabet $\frac{A}{1-q}$ is given by

$$
\sigma\left(\frac{A}{1-q} ; t\right):=\sum_{n \geq 0} S_{n}\left(\frac{A}{1-q}\right) t^{n}=\overleftarrow{\prod_{n \geq 0}} \sigma\left(q^{k} t\right)=\ldots \sigma\left(q^{3} t\right) \sigma\left(q^{2} t\right) \sigma(q t) \sigma(t)
$$

These symmetric functions are clearly noncommutative analogs of the commutative complete symmetric functions of the alphabet $\frac{A}{1-q}$.

Note 6.2 Relation (122) shows that $\sigma(A /(1-q) ; t)$ is a product of group-like elements for the coproduct $\Delta$. It follows that $\sigma(A /(1-q) ; t)$ is itself a group-like element for $\Delta$. That is,

$$
\Delta\left(S_{n}\left(\frac{A}{1-q}\right)\right)=\sum_{i=0}^{n} S_{i}\left(\frac{A}{1-q}\right) \otimes S_{n-i}\left(\frac{A}{1-q}\right) .
$$

We now give an expression of $S_{n}\left(\frac{A}{1-q}\right)$ in terms of ribbon functions. To this purpose, let us introduce the noncommutative symmetric functions $K_{n}(q)$ defined by

$$
K_{n}(q)=\sum_{|I|=n} q^{\operatorname{maj}(I)} R_{I}(A)
$$

where maj $(I)$ denotes the common major index of the permutations in the descent class corresponding to $R_{I}$. As shown in [12], one has the following formula.

Proposition 6.3 For every $n \geq 0$, one has

$$
S_{n}\left(\frac{A}{1-q}\right)=\frac{K_{n}(q)}{(q)_{n}}
$$

where $(q)_{n}$ denotes the product $(1-q)\left(1-q^{2}\right) \ldots\left(1-q^{n}\right)$.

One can also find in [12] the explicit expansion of $S_{n}\left(\frac{A}{1-q}\right)$ over the basis $S^{I}$ of noncommutative complete symmetric functions. 
Proposition 6.4 For every $n \geq 0$, one has

$$
S_{n}\left(\frac{A}{1-q}\right)=\sum_{|I|=n} \frac{q^{\operatorname{maj}(I)}}{\left(1-q^{i_{1}}\right)\left(1-q^{i_{1}+i_{2}}\right) \ldots\left(1-q^{i_{1}+\ldots+i_{r}}\right)} S^{I}
$$

where the sum is taken over all compositions $I=\left(i_{1}, \ldots, i_{r}\right)$ of weight $n$.

Let us now consider the automorphism $\beta_{q}$ of Sym defined by

$$
\beta_{q}\left(S_{n}(A)\right)=S_{n}\left(\frac{A}{1-q}\right)
$$

for all $n \geq 0$. Applying $\beta_{q}$ to (106) shows that

$$
\beta_{q}\left(S_{n}((1-q) A)\right)=S_{n}(A)
$$

for every $n \geq 0$, therefore justifying the claim that the transformations $A \rightarrow(1-q) A$ and $A \rightarrow A /(1-q)$ are mutually inverse.

Using the automorphism $\beta_{q}$, one can translate all the results obtained in Section 5.6 in terms of symmetric functions of the alphabet $A /(1-q)$.

Proposition 6.5 For all $n \geq 1$,

$$
S_{n}(A)=(1-q)\left(\sum_{|I|=n}(-1)^{\ell(I)-1} q^{n-i_{r}}\left[i_{r}\right]_{q} S^{I}\left(\frac{A}{1-q}\right)\right),
$$

the sum being taken over all compositions $I=\left(i_{1}, \ldots, i_{r}\right)$ of $n$.

Proof - This formula is just the image under $\beta_{q}$ of the identity (105).

It is also interesting to have the expression of $S_{n}$ on the basis $K^{I}(q)$ of $\mathbf{S y m}$ which is defined as usual by

$$
K^{I}(q)=K_{i_{1}}(q) \ldots K_{i_{r}}(q)
$$

for every composition $I=\left(i_{1}, \ldots, i_{r}\right)$.

Corollary 6.6 For all $n \geq 1$,

$$
S_{n}(A)=\frac{1}{(q)_{n-1}} \sum_{|I|=n}(-1)^{\ell(I)-1} q^{n-i_{r}}\left[\begin{array}{c}
n-1 \\
i_{1}, \ldots, i_{r-1}, i_{r}-1
\end{array}\right]_{q} K^{I}(q)
$$

the sum being taken over all compositions $I=\left(i_{1}, \ldots, i_{r}\right)$ of $n$.

Proof - This is a consequence of the last proposition and of the following relation:

$$
\left(1-q^{i_{r}}\right) S^{I}\left(\frac{A}{1-q}\right)=\frac{1}{(q)_{n-1}}\left[\begin{array}{c}
n-1 \\
i_{1}, \ldots, i_{r-1}, i_{r}-1
\end{array}\right]_{q} K^{I}(q) .
$$




\subsection{Klyachko's idempotent}

Using rather $K_{n}(q)$, one can rewrite (123) as

$$
\Delta\left(K_{n}(q)\right)=\sum_{i=0}^{n}\left[\begin{array}{c}
n \\
i
\end{array}\right]_{q} K_{i}(q) \otimes K_{n-i}(q)
$$

It follows in particular from Theorem 3.1 that $K_{n}(q)$ is a Lie quasi-idempotent for every $q$ such that

$$
\left[\begin{array}{c}
n \\
i
\end{array}\right]_{q}=0
$$

for all $i \in[1, n-1]$. This is the case when we take $q=\zeta$ a primitive $n$-th root of the unity. To obtain an idempotent from the quasi-idempotent $K_{n}(\zeta)$, we need the following lemma ( [2], see also [12]):

Lemma 6.7 Let $\zeta$ be a primitive $n$-th root of unity. For every $n \geq 1$, one has

$$
K_{n}(q) * K_{n}(\zeta)=(q)_{n-1} K_{n}(\zeta)
$$

Since $(\zeta)_{n-1}=n$, the lemma shows that the element

$$
\kappa_{n}(\zeta)=\frac{1}{n} K_{n}(\zeta)
$$

is a Lie idempotent. This element has been introduced in [17], and is usually called Klyachko's idempotent.

According to the results of Section 3.4, the relation between $K_{n}(q)$ and Klyachko's idempotent suggests to introduce for every $n \geq 1$ the family $E_{\lambda}(K ; q), \lambda$ being a partition of $n$, defined by

$$
E_{\lambda}(K ; q)=\frac{1}{(q)_{n-1}} \sum_{\sigma(I)=L}(-1)^{\ell(I)-1} q^{n-i_{r}}\left[\begin{array}{c}
n-1 \\
i_{1}, \ldots, i_{r-1}, i_{r}-1
\end{array}\right]_{q} K^{I}(q),
$$

the sum being taken over all compositions $I$ which are permutations of $\lambda$.

Example 6.8 For $n=2$ and $n=3$,

$$
\begin{gathered}
E_{2}(K ; q)=\frac{1}{1-q} K_{2}(q), \quad E_{11}(K ; q)=\frac{-q}{1-q} K^{11}(q), \\
E_{3}(K ; q)=\frac{1}{(1-q)\left(1-q^{2}\right)} K_{3}(q), \\
E_{12}(K ; q)=\frac{-q^{2}}{(1-q)\left(1-q^{2}\right)} K^{21}(q)+\frac{-q}{(1-q)^{2}} K^{12}(q), \quad E_{111}(K ; q)=\frac{q^{2}}{(1-q)^{2}} K^{111}(q) .
\end{gathered}
$$

The elements $E_{\lambda}(K ; q)$ are constructed by the same process as in Section 3.4 from formula (128). In particular,

$$
S_{n}(A)=\sum_{\lambda \vdash n} E_{\lambda}(K ; q)
$$

It follows that when $q=\zeta$ is a primitive $n$-th root of unity, the $E_{\lambda}(K ; \zeta)$ form a complete set of orthogonal idempotents for the internal product (and thus provide a decomposition for the descent algebra $\Sigma_{n}$ with coefficients in the cyclotomic field $\mathbb{Q}(\zeta)$ ). 


\subsection{Other symmetric functions}

In this section, we give some explicit formulas for the different noncommutative symmetric functions of the alphabet $A /(1-q)$.

6.3.1 $\Lambda_{n}(A /(1-q))$

Proposition 6.9 For $n \geq 1$,

$$
\Lambda_{n}\left(\frac{A}{1-q}\right)=\sum_{|I|=n} \frac{(-1)^{n+\ell(I)}}{\left(1-q^{i_{1}}\right)\left(1-q^{i_{1}+i_{2}}\right) \ldots\left(1-q^{i_{1}+\ldots+i_{r}}\right)} S^{\bar{I}} .
$$

Proof - One has

$$
\frac{A}{1-q}=q \frac{A}{1-q} \oplus A
$$

Hence we get

$$
\lambda\left(\frac{A}{1-q} ; t\right)=\lambda(A ; t) \lambda\left(q \frac{A}{1-q} ; t\right)
$$

or equivalently

$$
\sigma(A ;-t) \lambda\left(\frac{A}{1-q} ; t\right)=\lambda\left(q \frac{A}{1-q} ; t\right)
$$

It follows that

$$
\left(q^{n}-1\right) \Lambda_{n}\left(\frac{A}{1-q}\right)=\sum_{i=1}^{n}(-1)^{i} S_{i}(A) \Lambda_{n-i}\left(\frac{A}{1-q}\right),
$$

which is a recursive definition of $\Lambda_{n}\left(\frac{A}{1-q}\right)$. An induction based on this last formula immediately shows that the coefficient of $\Lambda_{n}\left(\frac{A}{1-q}\right)$ on $S^{\bar{I}}$ with $I=\left(i_{1}, \ldots, i_{r}\right)$ is equal to

$$
\frac{(-1)^{i_{r}}}{q^{n}-1} \frac{(-1)^{n-i_{r}+(r-1)}}{\left(1-q^{i_{1}}\right) \ldots\left(1-q^{i_{1}+\ldots+i_{r-1}}\right)}=\frac{(-1)^{n+r}}{\left(1-q^{i_{1}}\right) \ldots\left(1-q^{i_{1}+\ldots+i_{r}}\right)}
$$

as desired.

As a corollary, we can explicit the substitution $q \rightarrow 1 / q$ on symmetric functions of the alphabet $\frac{A}{1-q}$.

Corollary 6.10 Let $F(A)$ be a noncommutative symmetric function. Then,

$$
\left.F\left(\frac{A}{1-q}\right)\right|_{q \rightarrow 1 / q}=\left(\tilde{\omega}\left(F\left(q \frac{A}{1-q}\right)\right)\right)^{*} .
$$

Proof - It suffices to prove the result for complete functions, and using Proposition 6.4, one can write

$$
\begin{aligned}
& \left.S_{n}\left(\frac{A}{1-q}\right)\right|_{q=1 / q}=\sum_{|I|=n} \frac{q^{-\operatorname{maj}(I)}}{\left(1-q^{-i_{1}}\right)\left(1-q^{-i_{1}-i_{2}}\right) \ldots\left(1-q^{-i_{1}-i_{2}-\ldots-i_{r}}\right)} S^{I} \\
= & q^{n}\left(\sum_{|I|=n} \frac{(-1)^{r}}{\left(1-q^{i_{1}}\right)\left(1-q^{i_{1}+i_{2}}\right) \ldots\left(1-q^{i_{1}+i_{2}+\ldots+i_{r}}\right)} S^{I}\right)=(-1)^{n} \Lambda_{n}\left(q \frac{A}{1-q}\right)^{*},
\end{aligned}
$$

according to Proposition 6.9 whence the result. 


\subsection{2 $\Psi_{n}(A /(1-q))$}

The following formula leads to a new interesting family of Lie idempotents.

Theorem 6.11 The noncommutative power sums of the first kind of the alphabet $A /(1-$ q) are given by

$$
\Psi_{n}\left(\frac{A}{1-q}\right)=\frac{1}{1-q^{n}} \sum_{|I|=n} \frac{(-1)^{\ell(I)-1}}{\left[\begin{array}{c}
n-1 \\
\ell(I)-1
\end{array}\right]_{q}} q^{\operatorname{maj}(I)-\left(\begin{array}{c}
\ell(I) \\
2
\end{array}\right)} R_{I}(A) .
$$

Proof - Note again that one has

$$
\frac{A}{1-q}=q \frac{A}{1-q} \oplus A
$$

It follows therefore from Proposition 4.2 that

$$
\psi\left(\frac{A}{1-q} ; t\right)=\sigma(A ; t)^{-1} \psi\left(q \frac{A}{1-q} ; t\right) \sigma(A ; t)+\psi(A ; t),
$$

from which we get

$$
\begin{gathered}
\sigma(A ; t) \psi\left(\frac{A}{1-q} ; t\right)=\psi\left(q \frac{A}{1-q} ; t\right) \sigma(A ; t)+\sigma(A ; t) \psi(A ; t) \\
=\psi\left(q \frac{A}{1-q} ; t\right) \sigma(A ; t)+\frac{d}{d t} \sigma(A ; t) .
\end{gathered}
$$

That is,

$$
n S_{n}(A)=\sum_{i=0}^{n-1} S_{i}(A) \Psi_{n-i}\left(\frac{A}{1-q}\right)-\sum_{i=0}^{n-1} q^{n-i} \Psi_{n-i}\left(\frac{A}{1-q}\right) S_{i}(A)
$$

for all $n \geq 1$. This can be rewritten as

$$
\Psi_{n}\left(\frac{A}{1-q}\right)=\frac{1}{1-q^{n}}\left(n S_{n}(A)+\sum_{i=1}^{n-1} q^{i} \Psi_{i}\left(\frac{A}{1-q}\right) S_{n-i}(A)-\sum_{i=1}^{n-1} S_{n-i}(A) \Psi_{i}\left(\frac{A}{1-q}\right)\right)
$$

which is a recursive definition of the functions $\Psi_{n}\left(\frac{A}{1-q}\right)$. Hence relation (134) is a characterization of these functions. To prove the theorem, it is therefore sufficient to show that the functions defined by (133) satisfy (134). Hence, from now on, we suppose that $\Psi_{n}\left(\frac{A}{1-q}\right)$ is given by (133) and we shall check that (134) holds.

First, the coefficients of $R_{n}(A)$ in both sides of (134) are equal, since

$$
n=\sum_{i=0}^{n-1} \frac{1}{q^{n-i}}-\sum_{i=0}^{n-1} q^{n-i} \frac{1}{q^{n-i}}=\sum_{i=0}^{n-1} \frac{1-q^{n-i}}{1-q^{n-i}} .
$$

Let now $I=\left(i_{1}, \ldots, i_{r}\right)$ be a composition of $n$ distinct from $(n)$. Let us denote by $a_{I}$ the coefficient of $R_{I}$ in

$$
\sum_{i=0}^{n-1} S_{i}(A) \Psi_{n-i}\left(\frac{A}{1-q}\right)
$$


In the same way, let us denote by $b_{I}$ the coefficient of $R_{I}$ in

$$
\sum_{i=0}^{n-1} q^{n-i} \Psi_{n-i}\left(\frac{A}{1-q}\right) S_{i}(A)
$$

To establish (134), we must show that $a_{I}=b_{I}$ under the previous hypothesis. But, using the multiplication formula for ribbon Schur functions, it is easy to see that

$$
a_{I}=\sum_{i=0}^{i_{1}-1} \frac{q^{\operatorname{maj}(I)-(r-1) i-\left(\begin{array}{c}
r \\
2
\end{array}\right)}}{1-q^{n-i}} \frac{(-1)^{r-1}}{\left[\begin{array}{c}
n-i-1 \\
r-1
\end{array}\right]_{q}}+\frac{q^{\operatorname{maj}(I)-(r-1) i_{1}-\left(\begin{array}{c}
r-1 \\
2
\end{array}\right)}}{1-q^{n-i_{1}}} \frac{(-1)^{r-2}}{\left[\begin{array}{c}
n-i_{1}-1 \\
r-2
\end{array}\right]_{q}} .
$$

In the same way, one has

$$
b_{I}=\sum_{i=0}^{i_{r}-1} q^{n-i} \frac{q^{\operatorname{maj}(I)-\left(\begin{array}{c}
r \\
2
\end{array}\right)}}{1-q^{n-i}} \frac{(-1)^{r-1}}{\left[\begin{array}{c}
n-i-1 \\
r-1
\end{array}\right]_{q}}+q^{n-i_{r}} \frac{q^{\operatorname{maj}(I)-\left(n-i_{r}\right)-\left(\begin{array}{c}
r-1 \\
2
\end{array}\right)}}{1-q^{n-i_{r}}} \frac{(-1)^{r-2}}{\left[\begin{array}{c}
n-i_{r}-1 \\
r-2
\end{array}\right]_{q}} .
$$

Observe now that we can write

$$
a_{I}=(-1)^{r-1} q^{\operatorname{maj}(I)-\left(\begin{array}{c}
r \\
2
\end{array}\right)} a\left(i_{1}, n, r\right) \text { and } b_{I}=(-1)^{r-1} q^{\text {maj }(I)-\left(\begin{array}{c}
r \\
2
\end{array}\right)} b\left(i_{r}, n, r\right)
$$

where we set

$$
\begin{aligned}
& a(k, n, r)=\sum_{i=0}^{k-1} \frac{q^{-(r-1) i}}{1-q^{n-i}} \frac{1}{\left[\begin{array}{c}
n-i-1 \\
r-1
\end{array}\right]_{q}}-\frac{q^{(r-1)(1-k)}}{1-q^{n-k}} \frac{1}{\left[\begin{array}{c}
n-k-1 \\
r-2
\end{array}\right]_{q}}, \\
& b(k, n, r)=\sum_{i=0}^{k-1} \frac{q^{n-i}}{1-q^{n-i}} \frac{1}{\left[\begin{array}{c}
n-i-1 \\
r-1
\end{array}\right]_{q}}-\frac{q^{r-1}}{1-q^{n-k}} \frac{1}{\left[\begin{array}{c}
n-k-1 \\
r-2
\end{array}\right]_{q}}
\end{aligned}
$$

The proof is therefore reduced to the verification that $a\left(i_{1}, n, r\right)=b\left(i_{r}, n, r\right)$. To this purpose, we first give a lemma showing that $a(k, n, r)$ and $b(k, n, r)$ do not depend on $k$.

Lemma 6.12 For all $k, n, r \geq 1$, one has

$$
a(k, n, r)=a(1, n, r) \quad \text { and } \quad b(k, n, r)=b(1, n, r)
$$

Proof - It is sufficient to show that

$$
a(k+1, n, r)-a(k, n, r)=0 \quad \text { and } \quad b(k+1, n, r)-b(k, n, r)=0
$$

for all $k \geq 1$. Note first that $a(k+1, n, r)-a(k, n, r)$ is equal to

$$
\begin{aligned}
& \frac{q^{-(r-1) k}}{1-q^{n-k}}\left(\frac{1}{\left[\begin{array}{c}
n-k-1 \\
r-1
\end{array}\right]_{q}}+\frac{q^{r-1}}{\left[\begin{array}{c}
n-k-1 \\
r-2
\end{array}\right]_{q}}-\frac{1-q^{n-k}}{\left(1-q^{n-k-1}\right)\left[\begin{array}{c}
n-k-2 \\
r-2
\end{array}\right]_{q}}\right) \\
= & \frac{q^{-(r-1) k}}{(q)_{n-k}}(q)_{n-k-r}(q)_{r-2}\left(\left(1-q^{r-1}\right)+q^{r-1}\left(1-q^{n-k-r+1}\right)-\left(1-q^{n-k}\right)\right)=0,
\end{aligned}
$$


as may easily be checked. On the other hand, one can compute $b(k+1, n, r)-b(k, n, r)$ which is equal to

$$
\begin{aligned}
& \frac{q^{n-k}}{\left(1-q^{n-k}\right)\left[\begin{array}{c}
n-k-1 \\
r-1
\end{array}\right]_{q}}-\frac{q^{r-1}}{\left(1-q^{n-k-1}\right)\left[\begin{array}{c}
n-k-2 \\
r-2
\end{array}\right]_{q}}+\frac{q^{r-1}}{\left(1-q^{n-k}\right)\left[\begin{array}{c}
n-k-1 \\
r-2
\end{array}\right]_{q}} \\
& =\frac{(q)_{r-2}(q)_{n-k-r}}{(q)_{n-k}}\left(q^{n-k}\left(1-q^{r-1}\right)-q^{r-1}\left(1-q^{n-k}\right)+q^{r-1}\left(1-q^{n-k-r+1}\right)\right)=0 .
\end{aligned}
$$

This ends the proof of the lemma.

According to the lemma, it is now sufficient to check that

$$
a(1, n, r)=b(1, n, r) .
$$

But this reduces to

$$
\frac{1}{\left(1-q^{n}\right)\left[\begin{array}{l}
n-1 \\
r-1
\end{array}\right]_{q}}-\frac{1}{\left(1-q^{n-1}\right)\left[\begin{array}{l}
n-2 \\
r-2
\end{array}\right]_{q}}=\frac{q^{n}}{\left(1-q^{n}\right)\left[\begin{array}{l}
n-1 \\
r-1
\end{array}\right]_{q}}-\frac{q^{r-1}}{\left(1-q^{n-1}\right)\left[\begin{array}{l}
n-2 \\
r-2
\end{array}\right]_{q}}
$$

itself equivalent to

$$
\frac{1}{\left[\begin{array}{l}
n-1 \\
r-1
\end{array}\right]_{q}}=\frac{1-q^{r-1}}{\left(1-q^{n-1}\right)\left[\begin{array}{l}
n-2 \\
r-2
\end{array}\right]_{q}}
$$

which is obviously true.

Note 6.13 Using this theorem, one can get rather tricky $q$-identities from Newton's formulas. For instance, the relation

$$
\Psi_{n}\left(\frac{A}{1-q}\right)=\sum_{i=0}^{n-1}(-1)^{i-1}(n-i) \Lambda_{i}\left(\frac{A}{1-q}\right) S_{n-i}\left(\frac{A}{1-q}\right)
$$

is easily seen to be equivalent to the identities

$$
(q)_{\ell(I)-1}(q)_{n-\ell(I)} q^{\operatorname{maj}(I)+\left(\begin{array}{c}
\ell(I) \\
2
\end{array}\right)}=\sum_{i=0}^{n-1}(-1)^{i-1}(n-i)\left[\begin{array}{c}
n \\
i
\end{array}\right]_{q} q^{\operatorname{maj}\left(I_{j}^{\sim}\right)+\operatorname{maj}\left(I_{n-j}^{\prime}\right)}
$$

for any composition $I$ of $n$, where $I_{j}$ (resp. $I_{j}^{\prime}$ ) denotes the composition of $j$ associated to the diagram formed by the first (resp. last) $j$ cells of the ribbon diagram of $I$.

Note 6.14 Proposition 6.11 shows in particular that

$$
\lim _{q \rightarrow 1}\left(1-q^{n}\right) \Psi_{n}\left(\frac{A}{1-q}\right)=\Phi_{n}(A) .
$$




\subsection{A one-parameter family of Lie idempotents}

The inverse image under $\alpha$ of the noncommutative symmetric function $\varphi_{n}(q)$ defined by

$$
\varphi_{n}(q)=\frac{1}{n} \sum_{|I|=n} \frac{(-1)^{\ell(I)-1}}{\left[\begin{array}{c}
n-1 \\
\ell(I)-1
\end{array}\right]_{q}} q^{\operatorname{maj}(I)-\left(\begin{array}{c}
\ell(I) \\
2
\end{array}\right)} R_{I}(A)
$$

is clearly a $q$-analog of Solomon's idempotent $e_{n}^{[1]}$ (the first Eulerian idempotent). The properties of the corresponding element $e_{n}^{[1]}(q)$ of the descent algebra have been discussed in [8]. We will show how to derive them from the results of the preceding section. According to Proposition 6.11,

$$
\varphi_{n}(q)=\frac{1-q^{n}}{n} \Psi_{n}\left(\frac{A}{1-q}\right) .
$$

It follows in particular from this relation that $\varphi_{n}(q)$ is a primitive element for $\Delta$ and hence encodes a Lie quasi-idempotent according to Theorem 3.1. In fact, $\varphi_{n}(q)$ is actually a Lie idempotent as shown by the following proposition.

Proposition 6.15 For all $n \geq 1$,

$$
\Psi_{n}\left(\frac{A}{1-q}\right) * \Psi_{n}=\frac{n}{1-q^{n}} \Psi_{n}
$$

Proof - One has

$$
\begin{gathered}
\psi\left(\frac{A}{1-q} ; t\right) * \psi(A ; t)=\mu\left[\lambda\left(\frac{A}{1-q} ;-t\right) \otimes \frac{d}{d t} \sigma\left(\frac{A}{1-q} ; t\right) *(1 \otimes \psi(A ; t)+\psi(A ; t) \otimes 1)\right] \\
=\frac{d}{d t} \sigma\left(\frac{A}{1-q} ; t\right) * \psi(A ; t)
\end{gathered}
$$

from which we get

$$
\Psi_{n}\left(\frac{A}{1-q}\right) * \Psi_{n}=n S_{n}\left(\frac{A}{1-q}\right) * \Psi_{n} .
$$

To compute this internal product, observe that

$$
\begin{aligned}
& \sigma\left(\frac{1-q^{N}}{1-q} A ; t\right) * \psi(A ; t)=\mu_{N}\left[\overleftarrow{0 \leq i \leq N-1}_{\bigotimes^{\prime}} \sigma\left(A ; q^{i} t\right) * \sum 1 \otimes \ldots \otimes 1 \otimes \psi(A ; t) \otimes 1 \otimes \ldots\right] \\
& =\sum_{n \geq 0}\left(\sum_{i=0}^{N-1}\left(q^{i}\right)^{n}\right) \Psi_{n}=\sum_{n \geq 0} \frac{1-q^{N}}{1-q^{n}} \Psi_{n} .
\end{aligned}
$$

Taking the limit for $N \rightarrow \infty$, we obtain

$$
S_{n}\left(\frac{A}{1-q}\right) * \Psi_{n}=\frac{1}{1-q^{n}} \Psi_{n},
$$

and the required identity follows from (140).

Observe now that (137) and (138) imply that

$$
\varphi_{n}(0)=\frac{1}{n} \Psi_{n}, \varphi_{n}(1)=\frac{1}{n} \Phi_{n} .
$$

The specialization of $\varphi_{n}(q)$ at roots of unity is given by the following well-known lemma. 
Lemma 6.16 Let $\zeta$ be a primitive $n$-th root of unity. For every $i \in[0, n-1]$, one has

$$
\left[\begin{array}{c}
n-1 \\
i
\end{array}\right]_{\zeta}=(-1)^{i} \zeta^{-\left(\begin{array}{c}
i+1 \\
2
\end{array}\right)}
$$

Proof - Let us give a proof using symmetric functions. It is a classical result (see $[21,19])$ that

$$
\Lambda_{i}\left(\frac{1-q^{n-1}}{1-q}\right)=q^{\left(\begin{array}{c}
i \\
2
\end{array}\right)}\left[\begin{array}{c}
n-1 \\
i
\end{array}\right]_{q}
$$

for $i \in[0, n-1]$. It follows that

$$
\Lambda_{i}\left(\frac{q-q^{n}}{1-q}\right)=q^{i+\left(\begin{array}{c}
i \\
2
\end{array}\right)}\left[\begin{array}{c}
n-1 \\
i
\end{array}\right]_{q}=q^{\left(\begin{array}{c}
i+1 \\
2
\end{array}\right)}\left[\begin{array}{c}
n-1 \\
i
\end{array}\right]_{q} .
$$

Taking now $q=\zeta$, we find

$$
1+x+\ldots+x^{n-1}=\prod_{i=1}^{n-1}\left(x-\zeta^{i}\right)=\sum_{i=0}^{n-1} x^{n-1-i}(-1)^{i} \zeta^{\left(\begin{array}{c}
i+1 \\
2
\end{array}\right)}\left[\begin{array}{c}
n-1 \\
i
\end{array}\right]_{\zeta}
$$

whence the result.

Thus,

$$
\varphi_{n}(\zeta)=\frac{1}{n} K_{n}(\zeta)=\kappa_{n}(\zeta)
$$

when $\zeta$ is a primitive $n$-th root of unity. Hence we have proved

Proposition 6.17 The family $\varphi_{n}(q)$ is a family of Lie idempotents interpolating between Dynkin's idempotent $\frac{1}{n} \Psi_{n}$ (obtained for $\left.q=0\right)$, Solomon's Eulerian idempotent $\frac{1}{n} \Phi_{n}$ (obtained for $q=1$ ) and Klyachko's idempotent $\kappa_{n}(\zeta)$ (obtained for $q=\zeta$ a primitive $n$-th root of unity).

Moreover, one can check that

$$
\lim _{q \rightarrow \pm \infty} \varphi_{n}(q)=\frac{1}{n} \Psi_{n}^{*}
$$

It is now possible to construct from these $q$-idempotents, $q$-analogs of the Garsia-Reutenauer idempotents, and in particular of the Eulerian idempotents.

Example 6.18 Setting for short $E_{\lambda}(q)=E_{\lambda}\left(\varphi_{n}(q)\right)$, we have for $n=3$

$$
\begin{aligned}
E_{(3)}(q) & =\frac{1}{3(1+q)}\left[(1+q) R_{3}-q R_{21}-R_{12}+(1+q) R_{111}\right] \\
E_{(21)}(q) & =\frac{1}{6(1+q)}\left[3(1+q) R_{3}-(1-q) R_{21}+(1-q) R_{12}-3(1+q) R_{111}\right] \\
E_{(111)}(q) & =\frac{1}{6}\left[R_{3}+R_{21}+R_{12}+R_{111}\right]
\end{aligned}
$$


and for $n=4$

$$
\begin{aligned}
E_{(4)}(q)= & \frac{1}{4\left(1+q+q^{2}\right)}\left[R_{4}-q^{2} R_{31}-q R_{22}-R_{13}\right. \\
& \left.+q^{2} R_{112}+q R_{121}-\left(1+q+q^{2}\right) R_{1111}\right] \\
E_{(31)}(q)= & \frac{1}{12(1+q)\left(1+q+q^{2}\right)}\left[4\left(1+2 q+2 q^{2}+q^{3}\right) R_{4}+\left(1+3 q^{2}+2 q^{3}\right) R_{31}\right. \\
& -3\left(1+q+q^{2}+q^{3}\right) R_{22}+\left(3+4 q+q^{2}-2 q^{3}\right) R_{211} \\
& +\left(2+3 q+q^{3}\right) R_{13}-\left(1+5 q+5 q^{2}+q^{3}\right) R_{121} \\
& \left.+\left(-2+q+4 q^{2}+3 q^{3}\right) R_{112}+4\left(1+q+q^{2}+q^{3}\right) R_{1111}\right] \\
& \frac{1}{8}\left[R_{4}-R_{31}+R_{22}-R_{211}-R_{13}\right. \\
E_{(22)}(q)= & \left.+R_{121}-R_{112}+R_{1111}\right] \\
E_{(211)}(q)= & \frac{1}{12(1+q)}\left[3(1+q) R_{4}+2 q R_{31}+(1+q) R_{22}-2 R_{211}\right. \\
& \left.+2 q R_{13}-(1+q) R_{121}-2 q R_{112}-3(1+q) R_{1111}\right] \\
& \frac{1}{4 !} \Psi^{1111}=\frac{1}{24} \sum_{|I|=4} R_{I} .
\end{aligned}
$$




\section{$7 \quad$ Symmetric functions of the alphabet $\frac{\mid 1-t}{1-q \mid} A$}

In the noncommutative case, there are two natural manners to define symmetric functions of the alphabet $((1-t) /(1-q)) A$. Indeed such a notation can mean either

$$
\left(\frac{1}{1-q} \hat{\times}(1-t)\right) A \quad \text { or } \quad\left((1-t) \hat{\times} \frac{1}{1-q}\right) A
$$

It appears that these two definitions are interesting. We devote this section to the study of the first case, the second case being studied in the next section.

\subsection{Complete functions}

Definition 7.1 The generating series of the complete symmetric functions of the alphabet $\frac{\mid 1-t}{1-q \mid} A$ is given by

$$
\sigma\left(\frac{\mid 1-t}{1-q \mid} A ; x\right):=\sum_{i \geq 0} S_{n}\left(\frac{\mid 1-t}{1-q \mid} A\right) x^{n}=\overleftarrow{\prod_{i \geq 0}}\left(\lambda\left(A ;-q^{i} t x\right) \sigma\left(A ; q^{i} x\right)\right)
$$

Proposition 7.2 For $n \geq 1$, one has

$$
S_{n}\left(\frac{\mid 1-t}{1-q \mid} A\right)=\sum_{|I|=n}(-1)^{\ell(I)} \frac{\left(t^{i_{1}}-q^{i_{1}}\right)\left(t^{i_{2}}-q^{i_{1}+i_{2}}\right) \ldots\left(t^{i_{r-1}}-q^{i_{1}+\ldots+i_{r-1}}\right)\left(t^{i_{r}}-1\right)}{\left(1-q^{i_{1}}\right)\left(1-q^{i_{1}+i_{2}}\right) \ldots\left(1-q^{i_{1}+\ldots+i_{r}}\right)} S^{I} .
$$

Proof - By definition,

$$
\frac{\mid 1-t}{1-q \mid} A=q \frac{1-t}{1-q} A \oplus(1-t) A
$$

It follows that

$$
\sigma\left(\frac{\mid 1-t}{1-q \mid} A ; x\right)=\sigma\left(q \frac{\mid 1-t}{1-q \mid} A ; x\right) \sigma((1-t) A ; x)
$$

from which we deduce the relation

$$
S_{n}\left(\frac{\mid 1-t}{1-q \mid} A\right)=\sum_{i=0}^{n} q^{i} S_{i}\left(\frac{\mid 1-t}{1-q \mid} A\right) S_{n-i}((1-t) A),
$$

which is clearly equivalent to

$$
\left(1-q^{n}\right) S_{n}\left(\frac{\mid 1-t}{1-q \mid} A\right)=\sum_{i=0}^{n-1} q^{i} S_{i}\left(\frac{\mid 1-t}{1-q \mid} A\right) S_{n-i}((1-t) A) .
$$

Using now Proposition 5.30, we deduce from this last equality that

$$
\left(1-q^{n}\right) S_{n}\left(\frac{\mid 1-t}{1-q \mid} A\right)=\sum_{i=0}^{n-1} \sum_{|J|=n-i}(-1)^{\ell(J)-1} q^{i} t^{n-i-j_{r}}\left(1-t^{j_{r}}\right) S_{i}\left(\frac{\mid 1-t}{1-q \mid} A\right) S^{J}(A)
$$


Reasoning now by induction from (147), we get that the coefficient $a_{I}$ of $S^{I}$ in $S_{n}\left(\frac{\mid 1-t}{1-q \mid} A\right)$ with $I=\left(i_{1}, \ldots, i_{r}\right)$ is equal to

$$
a_{I}=(-1)^{r-1} \frac{1-t^{i_{r}}}{1-q^{n}} b\left(i_{1}, \ldots, i_{r}\right)=(-1)^{r} \frac{t^{i_{r}}-1}{1-q^{n}} b\left(i_{1}, \ldots, i_{r}\right)
$$

where we set

$$
b\left(i_{1}, \ldots, i_{r}\right)=\sum_{k=0}^{r-1} q^{i_{1}+\ldots+i_{k}} t^{i_{k+1}+\ldots+i_{r-1}} \frac{\left(t^{i_{1}}-q^{i_{1}}\right) \ldots\left(t^{i_{r-1}}-q^{i_{1}+\ldots+i_{k-1}}\right)\left(t^{i_{k}}-1\right)}{\left(1-q^{i_{1}}\right) \ldots\left(1-q^{i_{1}+\ldots+i_{k}}\right)},
$$

this sum being equal to 1 for $r=1$. The conclusion will result from the following lemma.

Lemma 7.3 Let $\left(i_{1}, \ldots, i_{r}\right)$ be an arbitrary composition. Then,

$$
b\left(i_{1}, \ldots, i_{r}\right)=\frac{\left(t^{i_{1}}-q^{i_{1}}\right)\left(t^{i_{2}}-q^{i_{1}+i_{2}}\right) \ldots\left(t^{i_{r-1}}-q^{i_{1}+\ldots+i_{r-1}}\right)}{\left(1-q^{i_{1}}\right)\left(1-q^{i_{1}+i_{2}}\right) \ldots\left(1-q^{i_{1}+\ldots+i_{r-1}}\right)},
$$

this product being equal to 1 for $r=1$.

Proof - One can check that

$$
b\left(i_{1}, \ldots, i_{r+1}\right)=b\left(i_{1}, \ldots, i_{r}\right) t^{i_{r}}+q^{i_{1}+\ldots+i_{r}} \frac{\left(t^{i_{1}}-q^{i_{1}}\right) \ldots\left(t^{i_{r-1}}-q^{i_{1}+\ldots+i_{r-1}}\right)\left(t^{i_{r}}-1\right)}{\left(1-q^{i_{1}}\right) \ldots\left(1-q^{i_{1}+\ldots+i_{r}}\right)} .
$$

By induction on $r$, it is therefore sufficient to verify that one has

$$
\begin{gathered}
t^{i_{r}} \frac{\left(t^{i_{1}}-q^{i_{1}}\right) \ldots\left(t^{i_{r-1}}-q^{i_{1}+\ldots+i_{r-1}}\right)}{\left(1-q^{i_{1}}\right) \ldots\left(1-q^{i_{1}+\ldots+i_{r-1}}\right)}+q^{i_{1}+\ldots+i_{r}} \frac{\left(t^{i_{1}}-q^{i_{1}}\right) \ldots\left(t^{i_{r-1}}-q^{i_{1}+\ldots+i_{r-1}}\right)\left(t^{i_{r}}-1\right)}{\left(1-q^{i_{1}}\right) \ldots\left(1-q^{i_{1}+\ldots+i_{r}}\right)} \\
=\frac{\left(t^{i_{1}}-q^{i_{1}}\right) \ldots\left(t^{i_{r}}-q^{i_{1}+\ldots+i_{r}}\right)}{\left(1-q^{i_{1}}\right) \ldots\left(1-q^{i_{1}+\ldots+i_{r}}\right)} .
\end{gathered}
$$

One can also give the expression of $S_{n}\left(\frac{\mid 1-t}{1-q \mid} A\right)$ on the ribbon Schur functions. To this purpose, we need some definitions. Let $I$ be a composition. One can write in a unique way

$$
I=H_{1} \triangleright H_{2} \triangleright \ldots \triangleright H_{r}
$$

where every $H_{i}=\left(1^{x} y\right)$ is a hook composition. The hook decomposition of $I$ is then the sequence $H D(I)=\left(H_{1}, H_{2}, \ldots, H_{r}\right)$. The hook composition $H C(I)$ associated with $I$ is the composition

$$
H C(I)=\left(\ell\left(H_{1}\right), \ell\left(H_{2}\right), \ldots, \ell\left(H_{r}\right)\right)
$$

obtained by taking the lengths of the hooks involved in the hook decomposition of $I$. Let for instance $I=(3,1,1,4,2)$.

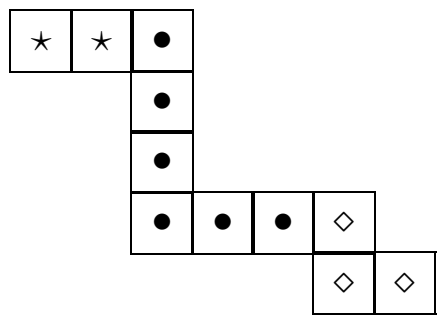


Then its hook decomposition and composition are respectively equal to

$$
H D(I)=\left(2,1^{3} 3,1^{1} 2\right) \text { and } H C(I)=(2,6,3) .
$$

We also associate with every composition $I$ a polynomial $H P_{I}(t, q)$ that we call the hook polynomial of $I$. This polynomial is constructed in the following way :

- draw the ribbon diagram $\Theta_{I}$ of $I$;

- reading $\Theta_{I}$ from left to right, write a cross in the first box of $\Theta_{I}$ and in the first two boxes of each hook (beginning with the second one) of the hook decomposition of $I$

- reading again $\Theta_{I}$ from left to right, number all boxes of $\Theta_{I}$ from 0 to $|I|-1$, without writing the corresponding number in a crossed box.

We obtain in this way a sequence $\left(i_{1}, i_{2}, \ldots, i_{m}\right)$ of positive integers in $[1,|I|-1]$ numbering all non-crossed boxes of $\Theta_{I}$. The hook polynomial $H P_{I}(t, q)$ is by definition the product of the factors

$$
q^{i_{j}}-t \quad \text { or } 1-q^{i_{j}} t
$$

according to whether the integer $i_{j}$ lies in the column part or in the row part of a hook involved in the hook decomposition of $I$ (the box located at the intersection of the row and the column of a hook is here considered as belonging to its column part). To give an example, let us consider again the composition $I=(3,1,4,2)$. The above procedure leads to

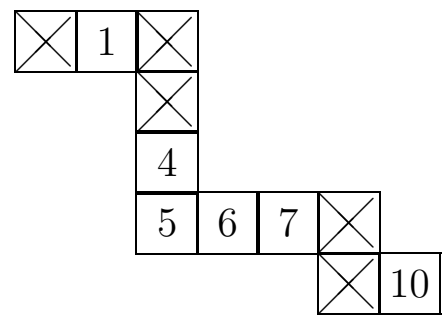

and the associated hook polynomial is

$$
H P_{3142}(t, q)=(1-q t)\left(q^{4}-t\right)\left(q^{5}-t\right)\left(1-q^{6} t\right)\left(1-q^{7} t\right)\left(1-q^{10} t\right) .
$$

We can now introduce the noncommutative symmetric function $K_{n}(t, q)$.

Definition 7.4 For every $n \geq 0$, we define $K_{n}(t, q)$ by setting

$$
K_{n}(t, q)=\sum_{|I|=n} q^{\operatorname{maj}(H C(I))}(1-t)^{\ell(H C(I))}(q-t)^{\ell(H C(I))-1} H P_{I}(t, q) R_{I}(A) .
$$

Note 7.5 Observe that one has the specializations

$$
\begin{gathered}
K_{n}(t, 0)=(1-t) \Theta_{n}(t), K_{n}(0, q)=K_{n}(q) \\
K_{n}(t, 1)=(1-t)^{n} \sum_{|I|=n} R_{I}(A)=(1-t)^{n}\left(S_{1}\right)^{n}, K_{n}(1, q)=0 .
\end{gathered}
$$

We can now give the decomposition of $S_{n}\left(\frac{1-t}{1-q} A\right)$ on ribbon functions. 
Theorem 7.6 For every $n \geq 1$, one has

$$
S_{n}\left(\frac{\mid 1-t}{1-q \mid} A\right)=\frac{K_{n}(t, q)}{(q)_{n}}
$$

where $(q)_{n}=(1-q)\left(1-q^{2}\right) \ldots\left(1-q^{n}\right)$.

Proof - Using formula (146) and Proposition 5.2, we get

$$
\left(1-q^{n}\right) S_{n}\left(\frac{\mid 1-t}{1-q \mid} A\right)=(1-t) \sum_{i=0}^{n-1} q^{i} S_{i}\left(\frac{\mid 1-t}{1-q \mid} A\right)\left(\sum_{k=0}^{n-i-1}(-t)^{k} R_{1^{k}, n-i-k}\right) .
$$

Using this relation, we shall prove the theorem by induction on $n$. Let $I$ be a composition of $n$ and let

$$
H D(I)=\left(H_{1}, \ldots, H_{r-1}, 1^{x} y\right)
$$

be its hook decomposition. Let us set $m=n-x-y$. Relation (153) and the product formula for ribbon functions show that the coefficient $a_{I}$ of $R_{I}$ in $S_{n}\left(\frac{\mid 1-t}{1-q \mid} A\right)$ is given by

$$
a_{I}=\frac{1-t}{1-q^{n}} b_{I}
$$

where $b_{I}$ is equal to

$$
\begin{aligned}
& \sum_{k=0}^{x} \frac{q^{\operatorname{maj}\left(H C\left(I_{m+k}\right)\right)}(1-t)^{\ell\left(H C\left(I_{m+k}\right)\right)}(q-t)^{\ell\left(H C\left(I_{m+k}\right)\right)-1}}{(q)_{m+k}} H P_{I_{m+k}}(t, q) q^{m+k}(-t)^{x-k} \\
+ & \sum_{k=1}^{y-1} \frac{q^{\operatorname{maj}\left(H C\left(I_{m+x+k}\right)\right)}(1-t)^{\ell\left(H C\left(I_{m+x+k}\right)\right)}(q-t)^{\ell\left(H C\left(I_{m+x+k}\right)\right)-1}}{(q)_{m+x+k}} H P_{I_{m+x+k}}(t, q) q^{m+x+k},
\end{aligned}
$$

$I_{k}$ denoting the composition whose ribbon diagram consists in the first $k$ boxes of the diagram of $I$. We shall distinguish two cases. Suppose first that $r=1$, i.e. that $I=1^{x} y$. In this case, $b_{I}=b(x, y)$ where

$$
b(x, y)=(-t)^{x}+\sum_{k=1}^{x} \frac{(1-t)}{(q)_{k}} H P_{1^{k}}(t, q) q^{k}(-t)^{x-k}+\sum_{k=1}^{y-1} \frac{(1-t)}{(q)_{x+k}} H P_{1^{x}, k}(t, q) q^{x+k} .
$$

The evaluation of this expression will be given by the following two lemmas.

Lemma 7.7 For every $x \geq 0$, one has

$$
(-t)^{x}+\sum_{k=1}^{x} \frac{(1-t)}{(q)_{k}}(q-t) \ldots\left(q^{k-1}-t\right) q^{k}(-t)^{x-k}=\frac{(q-t) \ldots\left(q^{x}-t\right)}{(q)_{x}} .
$$

Proof - Let $a(x)$ denote the left-hand side of relation (154). We have

$$
a(x+1)=(-t) a(x)+(1-t) \frac{(q-t) \ldots\left(q^{x}-t\right)}{(q)_{x+1}} q^{x+1} .
$$

Then, the lemma follows by induction on $x$ since

$$
a(x+1)=\frac{(q-t) \ldots\left(q^{x}-t\right)}{(q)_{x+1}}\left((-t)\left(1-q^{x+1}\right)+(1-t) q^{x+1}\right)=\frac{(q-t) \ldots\left(q^{x+1}-t\right)}{(q)_{x+1}} .
$$


Lemma 7.8 For every $x \geq 0$ and $y \geq 1$,

$$
\frac{1}{(q)_{x}}+\sum_{k=1}^{y-1} \frac{(1-t)}{(q)_{x+k}}\left(1-t q^{x+1}\right) \ldots\left(1-t q^{x+k-1}\right) q^{x+k}=\frac{\left(1-t q^{x+1}\right) \ldots\left(1-t q^{x+y-1}\right)}{(q)_{x+y-1}}
$$

Proof - Denote by $a(x, y)$ the left-hand side of (155). We have

$$
a(x, y+1)=a(x, y)+\frac{(1-t)}{(q)_{x+y}}\left(1-t q^{x+1}\right) \ldots\left(1-t q^{x+y-1}\right) q^{x+y} .
$$

The lemma follows by induction on $y$ since

$$
\begin{gathered}
\frac{\left(1-t q^{x+1}\right) \ldots\left(1-t q^{x+y-1}\right)}{(q)_{x+y-1}}+\frac{(1-t)}{(q)_{x+y}}\left(1-t q^{x+1}\right) \ldots\left(1-t q^{x+y-1}\right) q^{x+y} \\
=\frac{\left(1-t q^{x+1}\right) \ldots\left(1-t q^{x+y-1}\right)}{(q)_{x+y}}\left(1-q^{x+y}+(1-t) q^{x+y}\right)=\frac{\left(1-t q^{x+1}\right) \ldots\left(1-t q^{x+y}\right)}{(q)_{x+y}} .
\end{gathered}
$$

It follows now from Lemmas 7.7 and 7.8 applied to the defining relation of $b(x, y)$ with our general induction hypothesis that

$$
b(x, y)=\frac{(q-t) \ldots\left(q^{x}-t\right)\left(1-t q^{x+1}\right) \ldots\left(1-t q^{x+y-1}\right)}{(q)_{x+y-1}}=\frac{H P_{1^{x}, y}(t, q)}{(q)_{x+y-1}}
$$

from which we immediately get

$$
a_{1^{x}, y}=\frac{(1-t) H P_{1^{x}, y}(t, q)}{(q)_{x+y}}
$$

as required. This ends therefore the proof in the case $r=1$.

We now consider the case $r>1$. In this situation, one has $x \geq 1$ and

$$
\begin{gathered}
b_{I}=q^{M-m}(1-t)^{L-1}(q-t)^{L-2} H(t, q)\left(\frac{q^{m}(-t)^{x}}{(q)_{m}}+\frac{\left(1-t q^{m}\right) q^{m+1}(-t)^{x-1}}{(q)_{m+1}}\right) \\
+q^{M}(1-t)^{L}(q-t)^{L-1}\left(\sum_{k=2}^{x} \frac{H P_{I_{m+k}}(t, q) q^{m+k}(-t)^{x-k}}{(q)_{m+k}}+\sum_{k=1}^{y-1} \frac{H P_{I_{m+x+k}}(t, q) q^{m+x+k}}{(q)_{m+x+k}}\right)
\end{gathered}
$$

where we set $M=\operatorname{maj}(H C(I)), L=\ell(H C(I))$ and $H(t, q)=H P_{I_{m}}$. A simple computation allows then to rewrite this last expression as

$$
\begin{gathered}
b_{I}=q^{M}(1-t)^{L-1}(q-t)^{L-1}\left(\frac{H(t, q)(-t)^{x-1}}{(q)_{m+1}}+\sum_{k=2}^{x} \frac{(1-t)}{(q)_{m+k}} H P_{I_{m+k}}(t, q) q^{m+k}(-t)^{x-k}\right. \\
\left.+\sum_{k=1}^{y-1} \frac{(1-t)}{(q)_{m+x+k}} H P_{I_{m+x+k}}(t, q) q^{m+x+k}\right) .
\end{gathered}
$$

To finish these calculations, we need two more lemmas. 
Lemma 7.9 For every $x \geq 1$, one has

$$
\begin{gathered}
\frac{(-t)^{x-1}}{(q)_{m+1}}+\sum_{k=2}^{x} \frac{(1-t)}{(q)_{m+k}}\left(q^{m+2}-t\right) \ldots\left(q^{m+k-1}-t\right) q^{m+k}(-t)^{x-k} \\
=\frac{\left(q^{m+2}-t\right) \ldots\left(q^{m+x}-t\right)}{(q)_{m+x}} .
\end{gathered}
$$

Proof - Let $a(x)$ denote the left-hand side of (156). We have

$$
a(x+1)=a(x)(-t)+\frac{(1-t)}{(q)_{m+x+1}}\left(q^{m+2}-t\right) \ldots\left(q^{m+x}-t\right) q^{m+x+1} .
$$

The lemma follows now by induction on $x$ since one has

$$
\begin{gathered}
\frac{\left(q^{m+2}-t\right) \ldots\left(q^{m+x}-t\right)}{(q)_{m+x}}(-t)+\frac{(1-t)}{(q)_{m+x+1}}\left(q^{m+2}-t\right) \ldots\left(q^{m+x}-t\right) q^{m+x+1} \\
=\frac{\left(q^{m+2}-t\right) \ldots\left(q^{m+x}-t\right)}{(q)_{m+x+1}}\left((-t)\left(1-q^{m+x+1}\right)+q^{m+x+1}(1-t)\right) \\
=\frac{\left(q^{m+2}-t\right) \ldots\left(q^{m+x+1}-t\right)}{(q)_{m+x+1}}
\end{gathered}
$$

as required.

Lemma 7.10 For every $x \geq 1$ and $y \geq 1$, one has

$$
\begin{gathered}
\frac{1}{(q)_{m+x}}+\sum_{k=1}^{y-1} \frac{(1-t)}{(q)_{m+x+k}}\left(1-t q^{m+x+1}\right) \ldots\left(1-t q^{m+x+k-1}\right) q^{m+x+k} \\
=\frac{\left(1-t q^{m+x+1}\right) \ldots\left(1-t\left(1-t q^{m+x+y-1}\right)\right.}{(q)_{m+x+y-1}}
\end{gathered}
$$

Proof - Let $a(x, y)$ denote the left-hand side of (157). One has

$$
a(x, y+1)=a(x, y)+\frac{(1-t)}{(q)_{m+x+y}}\left(1-t q^{m+x+1}\right) \ldots\left(1-t q^{m+x+y-1}\right) q^{m+x+y} .
$$

The lemma follows now by induction on $y$ since

$$
\begin{gathered}
\frac{\left(1-t q^{m+x+1}\right) \ldots\left(1-t q^{m+x+y-1}\right)}{(q)_{m+x+y-1}}+\frac{(1-t)}{(q)_{m+x+y}}\left(1-t q^{m+x+1}\right) \ldots\left(1-t q^{m+x+y-1}\right) q^{m+x+y} \\
=\frac{\left(1-t q^{m+x+1}\right) \ldots\left(1-t q^{m+x+y-1}\right)}{(q)_{m+x+y}}\left(1-q^{m+x+y}+q^{m+x+y}(1-t)\right) \\
=\frac{\left(1-t q^{m+x+1}\right) \ldots\left(1-t q^{m+x+y}\right)}{(q)_{m+x+y}},
\end{gathered}
$$

as required. 
It follows then from Lemmas 7.9 and 7.10 and from the expression of $b_{I}$ already obtained that

$$
b_{I}=q^{M}(1-t)^{L-1}(q-t)^{L-1} \frac{H(t, q)\left(q^{m+2}-t\right) \ldots\left(q^{m+x}-t\right)\left(1-t q^{m+x+1}\right) \ldots\left(1-t q^{n-1}\right)}{(q)_{n-1}} .
$$

Using now our general induction hypothesis, we deduce from this last expression that

$$
a_{I}=\frac{q^{M}(1-t)^{L}(q-t)^{L-1} H P_{I}(t, q)}{(q)_{n}}
$$

as required. This ends therefore the proof of Theorem 7.6.

Example 7.11 For $n=2$ and $n=3$, we have

$$
\begin{gathered}
S_{2}\left(\frac{\mid 1-t}{1-q \mid} A\right)=\frac{(1-t)(1-t q) R_{2}(A)+(1-t)(q-t) R_{11}(A)}{(1-q)\left(1-q^{2}\right)} \\
S_{3}\left(\frac{\mid 1-t}{1-q \mid} A\right)=\frac{(1-t)(1-t q)\left(1-t q^{2}\right) R_{3}(A)+q(1-t)^{2}(q-t) R_{21}(A)}{(1-q)\left(1-q^{2}\right)\left(1-q^{3}\right)} \\
+\frac{(1-t)(q-t)\left(1-t q^{2}\right) R_{12}(A)+(1-t)(q-t)\left(q^{2}-t\right) R_{111}(A)}{(1-q)\left(1-q^{2}\right)\left(1-q^{3}\right)} .
\end{gathered}
$$

\subsection{Eulerian idempotents associated to Dynkin's projectors}

As can be observed on Example 3.20, the Eulerian-like idempotents $E_{n}^{[k]}(\Psi)$ associated by the construction of Section 3.4 to the sequence $\left(\Psi_{n}\right)$ (which are also the specialization $q=0$ of the $q$-Eulerians $\left.E_{n}^{[k]}(q)\right)$ appear to possess an interesting generating function. Its expression on the ribbon basis can be obtained by an appropriate specialization of Theorem 7.6.

Theorem 7.12 Let $x$ be an indeterminate. Then,

$$
\left.\sum_{r=0}^{n} x^{r} E_{n}^{[r]}(\Psi)=\lim _{q \rightarrow 1} S_{n}\left(\frac{1-q^{x} \mid}{\mid 1-q} A\right)\right)
$$

Proof - By definition, one has

$$
\begin{gathered}
\sigma\left(\frac{1-q^{x} \mid}{\mid 1-q} A ; 1\right)=\overleftarrow{\prod_{k \geq 0}} \sigma\left(\left(1-q^{x}\right) A ; q^{k}\right) \\
=\sum_{r \geq 0} \sum_{\ell(I)=r} \frac{q^{\operatorname{maj}(I)}}{\left(1-q^{i_{1}}\right)\left(1-q^{i_{1}+i_{2}}\right) \cdots\left(1-q^{\left.i_{1}+\cdots+i_{r}\right)}\right.} S^{I}\left(\left(1-q^{x}\right) A\right) \\
=\sum_{r \geq 0} \sum_{\ell(I)=r}\left(\frac{1-q^{x}}{1-q}\right)^{r} \frac{q^{\operatorname{maj}(I)}}{\left[i_{1}\right]_{q}\left[i_{1}+i_{2}\right]_{q} \cdots\left[i_{1}+\cdots+i_{r}\right]_{q}} \Theta^{I}\left(q^{x}\right)
\end{gathered}
$$


and for $q \rightarrow 1$, this expression tends to

$$
\sum_{I} x^{\ell(I)} \frac{\Psi^{I}}{i_{1}\left(i_{1}+i_{2}\right) \cdots\left(i_{1}+\cdots+i_{r}\right)},
$$

as required.

Taking now the limit for $q \rightarrow 1$ in Theorem 7.6, we arrive at the following description of the generating function

$$
E_{n}(\Psi ; x)=\sum_{k} x^{k} E_{n}^{[k]}(\Psi) .
$$

Associate to a composition $I$ its hook decomposition and the sequence $H C(I)$ as in subsection 7.1, and define a polynomial $H_{I}(x)$ as the product of the factors

$$
x-i_{j} \quad \text { or } \quad x+i_{j}
$$

according to whether the integer $i_{j}$ lies in the column or in the row part of a hook involved in the hook decomposition of $I$. For instance,

$$
H_{3142}=(x+1)(x-4)(x-5)(x+6)(x+7)(x+10) .
$$

Thus, we obtain :

\section{Corollary 7.13}

$$
E_{n}(\Psi ; x)=\frac{1}{n !} \sum_{|I|=n} x^{\ell(H C(I))}(x-1)^{\ell(H C(I))-1} H_{I}(x) \cdot R_{I} .
$$

\subsection{A $t$-analog of Klyachko's idempotent}

Another family of Lie idempotents can be obtained from Theorem 7.6. These results obtained in collaboration with G. Duchamp, have been announced in [8].

Using the same argument as in Section 6.2, we obtain

$$
\Delta\left(K_{n}(t, q)\right)=\sum_{i=0}^{n}\left[\begin{array}{c}
n \\
i
\end{array}\right]_{q} K_{i}(t, q) \otimes K_{n-i}(t, q) .
$$

It follows in the same way that $K_{n}(t, \zeta)$ is a Lie quasi-idempotent when $\zeta$ is a primitive $n$-th root of unity. The normalization factor of $K_{n}(t, \zeta)$ is given by the following result.

Proposition 7.14 For every $n \geq 1$, one has

$$
K_{n}(t, q) * \Phi_{n}=(q)_{n-1}\left(1-t^{n}\right) \Phi_{n}
$$

Proof - Let $s_{N}(t, q)$ be the series defined by

$$
s_{N}(t, q)=\left(\lambda\left(-q^{N-1} t\right) \sigma\left(q^{N-1}\right)\right)\left(\lambda\left(-q^{N-2} t\right) \sigma\left(q^{N-2}\right)\right) \ldots(\lambda(-t) \sigma(1)) .
$$

Then one has

$$
s_{N}(t, q) * \Phi_{n}=\mu_{2 N}\left[\left(\lambda\left(-q^{N-1} t\right) \otimes \sigma\left(q^{N-1}\right) \otimes \ldots \otimes \lambda(-t) \otimes \sigma(1)\right) * \Delta^{2 N}\left(\Phi_{n}\right)\right]
$$




$$
=\left(\sum_{i=0}^{N-1}\left(\left(q^{i}\right)^{n}+t^{n}\left(-q^{i}\right)^{n}(-1)^{n-1}\right)\right) \Phi_{n}=\left(1-t^{n}\right)\left(\sum_{i=0}^{N-1}\left(q^{n}\right)^{i}\right) \Phi_{n}
$$

using the primitivity of $\Phi_{n}$ and the fact that $\Lambda_{n} * \Phi_{n}=\omega\left(\Phi_{n}\right)=(-1)^{n-1} \Phi_{n}$. Taking the limit for $N \rightarrow+\infty$, we obtain

$$
\sigma\left(\frac{1-t}{1-q} A\right) * \Phi_{n}=\frac{1-t^{n}}{1-q^{n}} \Phi_{n}
$$

The result follows now from this equality and Proposition 7.6.

As a consequence of the last proposition, we see that the element

$$
\kappa_{n}(t, \zeta)=\frac{1}{n\left(1-t^{n}\right)} K_{n}(t, \zeta)
$$

is a Lie idempotent when $\zeta$ is a primitive $n$-th root of unity. Note that this idempotent reduces to Klyachko's idempotent for $t=0$. 


\section{Symmetric functions of the alphabet $\frac{1-t \mid}{\mid 1-q} A$}

\subsection{Complete functions}

The alphabet $\frac{1-t \mid}{\mid 1-q} A$ is defined by setting

$$
\frac{1-t \mid}{\mid 1-q} A=(1-t) \hat{\times} \frac{A}{1-q}=\left((1-t) \hat{\times} \frac{1}{1-q}\right) A .
$$

This leads to the following definition of complete functions of this alphabet.

Definition 8.1 The complete functions of the alphabet $\frac{1-t \mid}{\mid 1-q} A$ are defined by the following generating series :

$$
\sigma\left(\frac{1-t \mid}{\mid 1-q} A\right):=\sum_{n \geq 0} S_{n}\left(\frac{1-t \mid}{\mid 1-q} A\right) x^{n}=\prod_{i \geq 0} \lambda\left(A ;-t q^{i} x\right) \overleftarrow{\prod_{i \geq 0}} \sigma\left(A ; q^{i} x\right)
$$

Proposition 8.2 For every $n \geq 1$, one has

$$
S_{n}\left(\frac{1-t \mid}{\mid 1-q} A\right)=\sum_{|I|=n} \frac{q^{\operatorname{maj}(I)-\left(\begin{array}{c}
\ell(I) \\
2
\end{array}\right)}}{(q)_{n}}(t-1) \prod_{i=1}^{n-\ell(I)}\left(t q^{i}-1\right) \prod_{i=1}^{\ell(I)-1}\left(t-q^{i}\right) R_{I},
$$

where we set $(q)_{n}=(1-q) \ldots\left(1-q^{n}\right)$.

The proof, which proceeds by induction as in the preceding section, is left to the reader.

An interesting consequence of Proposition 8.2 is the obtention of another $t$-analogue of Klyachko's idempotent. Using the same technique as in section 7.3, one can prove that the element

$$
\kappa_{n}^{\prime}(t, \zeta)=\frac{K_{n}^{\prime}(t, \zeta)}{n\left(1-t^{n}\right)}
$$

where we set

$$
K_{n}^{\prime}(t, q)=\sum_{|I|=n} q^{\operatorname{maj}(I)-\left(\begin{array}{c}
\ell(I) \\
2
\end{array}\right)} \prod_{i=1}^{n-\ell(I)}\left(t q^{i}-1\right) \prod_{i=1}^{\ell(I)-1}\left(t-q^{i}\right) R_{I}
$$

is a Lie idempotent when $\zeta$ is a primitive $n$-th root of unity. Clearly, this idempotent reduce to Klyachko's idempotent for $t=0$.

\subsection{Symmetric functions of the alphabet $\frac{1-q^{n} \mid}{\mid 1-q} A$}

The definition of complete functions of the alphabet $\frac{A}{1-q}$ suggests to define complete functions of the alphabet $\frac{1-q^{n}}{1-q} A$ as follows, in order that $\frac{1-q^{n}}{1-q} A \rightarrow A /(1-q)$ for $n \rightarrow \infty$.

Definition 8.3 The generating series of the complete symmetric functions of the alphabet $\left(\left(1-q^{n}\right) /(1-q)\right) A$ is given by

$$
\sigma\left(\frac{1-q^{n}}{1-q} A ; t\right):=\sum_{i \geq 0} S_{n}\left(\frac{1-q^{n}}{1-q} A\right) t^{n}=\sigma\left(A ; q^{n-1} t\right) \ldots \sigma(A ; q t) \sigma(A ; t) \text {. }
$$


We now restate our definition using basic operations on ordered alphabets.

\section{Proposition 8.4}

$$
\frac{1-q^{n}}{1-q} A=\left(1-q^{n}\right) \hat{\times} \frac{A}{1-q}=\left(\left(1-q^{n}\right) \hat{\times} \frac{1}{1-q}\right) A=\frac{1-q^{n} \mid}{\mid 1-q} A .
$$

Proof - For $k \geq n$, let us set

$$
\sigma_{k}(t)=\sigma\left(A ; q^{k n+n-1} t\right) \ldots \sigma\left(A ; q^{k n+1} t\right) \sigma\left(A ; q^{k n} t\right) .
$$

It is easy to check that

$$
\sigma_{k}(t)=\sum_{i \geq 0} q^{k n i} S_{i}\left(\frac{1-q^{n}}{1-q} A\right) t^{i}
$$

for every $k \geq 0$. On the other hand, we clearly have

$$
\sigma\left(\frac{A}{1-q} ; t\right)=\overleftarrow{\prod_{k \geq 0}} \sigma_{k}(t)=\ldots \sigma_{2}(t) \sigma_{1}(t) \sigma_{0}(t)
$$

Using relation (166) and the same method as in [12], one obtains

$$
S_{k}\left(\frac{A}{1-q}\right)=\sum_{|I|=k} \frac{q^{n \text { maj }(I)}}{\left(1-q^{n i_{1}}\right)\left(1-q^{n\left(i_{1}+i_{2}\right)}\right) \ldots\left(1-q^{n\left(i_{1}+\ldots+i_{r}\right)}\right)} S^{I}\left(\frac{1-q^{n}}{1-q} A\right) .
$$

It follows from this identity and from (106) that the transition matrix from the basis $S^{I}\left(\frac{A}{1-q}\right)$ to the basis $S^{I}\left(\frac{1-q^{n}}{1-q} A\right)$ is equal to the transition matrix from the basis $S^{I}(A)$ to the basis $S^{I}\left(\left(1-q^{n}\right) A\right)$. The same property holds for the inverse transition matrices. Hence relation (105) gives

$$
S_{k}\left(\frac{1-q^{n}}{1-q} A\right)=\left(1-q^{n}\right) \sum_{|I|=k}(-1)^{\ell(I)-1} q^{n\left(k-i_{r}\right)}\left[i_{r}\right]_{q^{n}} S^{I}\left(\frac{A}{1-q}\right)
$$

and the result follows from Proposition 5.30.

As a corollary of the proof, we have

Corollary 8.5 For every $k \geq 0$,

$$
S_{k}\left(\frac{1-q^{n}}{1-q} A\right)=\left(1-q^{n}\right) \sum_{|I|=k}(-1)^{\ell(I)-1} q^{n\left(k-i_{r}\right)}\left[i_{r}\right]_{q^{n}} S^{I}\left(\frac{A}{1-q}\right) .
$$

\subsubsection{Klyachko's idempotent again}

We can now give a new interpretation of Klyachko's idempotent.

Proposition 8.6 Let $\zeta$ be a primitive $n$-root of unity. Then,

$$
K_{n}(\zeta)=\left.S_{n}\left(\frac{1-q^{n} \mid}{\mid 1-q} A\right)\right|_{q=\zeta}
$$


Proof - For every composition $I=\left(i_{1}, i_{2}, \ldots, i_{r}\right)$ of $n$, we can write

$$
S^{I}\left(\frac{A}{1-q}\right)=\frac{K^{I}(q)}{(q)_{i_{1}}(q)_{i_{2}} \ldots(q)_{i_{r}}}
$$

Hence, when $I$ is not equal to $(n)$, we clearly have

$$
\left.\left(1-q^{n}\right) S^{I}\left(\frac{A}{1-q}\right)\right|_{q=\zeta}=\left.\frac{1-q^{n}}{(q)_{i_{1}}(q)_{i_{2}} \ldots(q)_{i_{r}}} K^{I}(q)\right|_{q=\zeta}=0
$$

since if this case all $(\zeta)_{i_{k}}$ are different from 0 . On the other hand,

$$
\left.\left(1-q^{n}\right) S_{n}\left(\frac{A}{1-q}\right)\right|_{q=\zeta}=\left.\frac{K_{n}(q)}{(1-q)\left(1-q^{2}\right) \ldots\left(1-q^{n-1}\right)}\right|_{q=\zeta}=\frac{1}{n} K_{n}(\zeta) .
$$

Applying now Corollary 8.5 we find

$$
\left.S_{n}\left(\frac{1-q^{n}}{1-q} A\right)\right|_{q=\zeta}=K_{n}(\zeta)
$$

\subsubsection{Other formulas}

Let $X_{q}(n)$ be the alphabet $\left\{1, q, \ldots q^{n-1}\right\}$ totally ordered by

$$
q^{n-1}>q^{n-2}>\ldots>q>1 .
$$

By definition, one has $\frac{1-q^{n}}{1-q} A=X_{q}(n) A$. It follows that

$$
S_{k}\left(\frac{1-q^{n}}{1-q} A\right)=\sum_{|I|=k} M_{I}\left(X_{q}(n)\right) S^{I}(A) .
$$

It seems unlikely that a closed formula for $M_{I}\left(X_{q}(n)\right)$ can be obtained. We shall however present several recursion relations allowing its evaluation.

Proposition 8.7 For every composition I, one has

$$
M_{I}\left(X_{q}(n)\right)=\left\{\begin{array}{cc}
q^{\text {maj }(I)} P_{I}^{(n-\ell(I))}(q) & \text { if } \ell(I) \leq n \\
0 & \text { if } \ell(I)>n
\end{array},\right.
$$

where $P_{I}^{(m)}(q)$ is a polynomial of $\mathbb{N}[q]$ recursively defined by

$$
P_{i_{1}}^{(m)}(q)=[m+1]_{q^{i_{1}}} \quad \text { and } \quad P_{I}^{(m)}=\sum_{k=0}^{m} q^{k|I|} P_{\left(i_{1}, \ldots, i_{r-1}\right)}^{\left(m-i_{1}\right)}(q),
$$

for every composition $I=\left(i_{1}, i_{2}, \ldots, i_{r}\right)$ and every nonnegative integer $m \in \mathbb{N}$. 
Proof - By definition,

$$
M_{I}\left(X_{q}(n)\right)=\sum_{n-1 \geq n_{1}>\ldots>n_{r} \geq 0} q^{n_{1} i_{1}+\ldots+n_{r r}},
$$

from which it follows that $M_{I}\left(X_{q}(n)\right)=0$ when $\ell(I)>n$. On the other hand, when $\ell(I) \leq n$, we can write

$$
M_{I}\left(X_{q}(n)\right)=q^{\operatorname{maj}(I)} P_{I}^{(n-\ell(I))}(q)
$$

where $P_{I}^{(m)}(q)$ denotes the polynomial of $\mathbb{N}[q]$ defined by

$$
P_{I}^{(m)}(q)=\sum_{m \geq n_{1} \geq \ldots \geq n_{r} \geq 0} q^{n_{1} i_{1}+\ldots+n_{r} i_{r}} .
$$

Hence it follows from this definition that $P_{i_{1}}^{(m)}(q)=[m+1]_{q^{i_{1}}}$. We can also write

$$
\begin{gathered}
P_{I}^{(m)}(q)=\sum_{k=0}^{m} q^{k i_{r}}\left(\sum_{m \geq n_{1} \geq \ldots \geq n_{r-1} \geq k} q^{n_{1} i_{1}+\ldots+n_{r-1} i_{r-1}}\right), \\
=\sum_{k=0}^{m} q^{k|I|}\left(\sum_{m-k \geq n_{1} \geq \ldots \geq n_{r-1} \geq 0} q^{n_{1} i_{1}+\ldots+n_{r-1} i_{r-1}}\right)=\sum_{k=0}^{m} q^{k|I|} P_{\left(i_{1}, \ldots, i_{r-1}\right)}^{(m-k)}(q) .
\end{gathered}
$$

Note 8.8 Using (171), one can obtain other recursive formulas for $P_{I}^{(m)}(q)$. For instance

$$
P_{I}^{(m)}(q)=\sum_{k=0}^{m} q^{k i_{1}} P_{\left(i_{1}+i_{2}, i_{3}, \ldots, i_{r}\right)}^{\left(m-i_{1}\right)}(q),
$$

and also

$$
P_{I}^{(m)}(q)=\frac{1}{1-q^{i_{1}}}\left(P_{\left(i_{1}+i_{2}, i_{3}, \ldots, i_{r}\right)}^{(m)}(q)-q^{m i_{1}} P_{\left(i_{2}, \ldots, i_{r}\right)}^{(m)}(q)\right) .
$$




\section{Lie quasi-idempotents as Lie polynomials}

The Lie elements of the descent algebra are in particular elements of the multilinear component $L_{\left(1^{n}\right)}([n])$ of the free Lie algebra over the alphabet $[n]=\{1, \ldots, n\}$. In this section, we give explicit expansions on a basis of multilinear alternants for some of these elements.

\subsection{Left derivative}

The left derivative $\Lambda_{1}^{-1}$ is the linear morphism of Sym defined on the basis $\Lambda^{I}$ by the relations

$$
\Lambda_{1}^{-1} \cdot \Lambda^{i_{1}, \ldots, i_{r}}=\left\{\begin{array}{cl}
\Lambda^{i_{2}, \ldots, i_{r}} & \text { if } i_{1}=1 \\
0 & \text { if } i_{1} \neq 1
\end{array} .\right.
$$

We note that $\Lambda_{1}^{-1}$ is characterized by the following three properties :

1) $\Lambda_{1}^{-1}$ is an additive morphism of Sym,

2) $\Lambda_{1}^{-1}$ satisfies to the property

$$
\Lambda_{1}^{-1} \cdot(F G)=\left(\Lambda_{1}^{-1} \cdot F\right) G+(F \mid 1) \Lambda_{1}^{-1} \cdot G
$$

for every $F, G \in S y m$, where $(F \mid 1)$ denotes the constant term of $F$.

3)

$$
\Lambda_{1}^{-1} \cdot \Lambda_{n}=\left\{\begin{array}{ll}
1 & \text { if } n=1 \\
0 & \text { if } n \neq 1
\end{array} .\right.
$$

One can describe the action of $\Lambda_{1}^{-1}$ on the usual bases of noncommutative symmetric functions.

Proposition 9.1 For $n \geq 1$ and for any composition $I=\left(i_{1}, \ldots, i_{r}\right)$,

$$
\begin{gathered}
\Lambda_{1}^{-1} \cdot S_{n}=S_{n-1}, \quad \Lambda_{1}^{-1} \cdot \Psi_{n}=S_{n-1}=\sum_{|I|=n-1} \frac{1}{\pi_{u}(I)} \Psi^{I}, \\
\Lambda_{1}^{-1} \cdot R_{I}=\left\{\begin{array}{cc}
R_{i_{1}-1, i_{2}, \ldots, i_{r}} & \text { if } i_{1} \neq 1 \\
0 & \text { if } i_{1}=1
\end{array} .\right.
\end{gathered}
$$

Proof - The expression of $\Lambda_{1}^{-1} \cdot R_{I}$ (and hence of $\Lambda_{1}^{-1} \cdot S_{n}$ ) is an immediate consequence of the fact that

$$
M(R, \Lambda)_{n}=\left(\begin{array}{cc}
-1 & 1 \\
1 & 0
\end{array}\right)^{\otimes(n-1)}
$$

In particular,

$$
\Lambda_{1}^{-1} \cdot \sigma(t)=t \sigma(t)
$$

On the other hand, using Eq. (173), one obtains

$$
\begin{aligned}
\Lambda_{1}^{-1} \cdot \psi(t) & \left.=\Lambda_{1}^{-1} \cdot\left(\lambda(-t) \frac{d}{d t} \sigma(t)\right)=\left(\Lambda_{1}^{-1} \cdot \lambda(-t)\right) \frac{d}{d t} \sigma(t)\right)+(\lambda(-t) \mid 1) \Lambda_{1}^{-1} \cdot \frac{d}{d t} \sigma(t) \\
& =(-t) \frac{d}{d t} \sigma(t)+\Lambda_{1}^{-1} \cdot \frac{d}{d t} \sigma(t)=(-t) \frac{d}{d t} \sigma(t)+\frac{d}{d t}\left(\Lambda_{1}^{-1} \cdot \sigma(t)\right) .
\end{aligned}
$$


Using now relation (177), we get

$$
\Lambda_{1}^{-1} \cdot \psi(t)=(-t) \frac{d}{d t} \sigma(t)+\frac{d}{d t}(t \sigma(t))=\sigma(t) .
$$

\subsection{Multilinear Lie polynomials}

Let $\pi$ be a Lie quasi-idempotent of $\mathbf{S y m}_{n}$. Let $\pi^{\prime}$ denote its inverse image by $\alpha$ in $\Sigma_{n}$. By hypothesis, $\pi^{\prime}$ is a Lie projector (up to a normalization) or a Lie nilpotent. This means that the image $l_{n}(\pi)$ of the standard word $12 \ldots n$ by $\pi^{\prime}$ is a standard Lie polynomial. That is,

$$
l_{n}(\pi)=12 \ldots n \cdot \pi^{\prime} \in L_{1^{n}}(\{1,2, \ldots, n\}),
$$

where $L_{1^{n}}(\{1,2, \ldots, n\})$ denotes the multihomogeneous component of multidegree $\left(1^{n}\right)$ of the free Lie algebra over the alphabet $\{1,2, \ldots, n\}$. Let us now give an explicit description of the Lie element $l_{n}(\pi)$.

Recall first that Witt's formulas imply that $L_{1^{n}}(\{1,2, \ldots, n\})$ is a free module of dimension $(n-1)$ !. It follows from this property that the family

$$
[\sigma]=[[\ldots[[1, \sigma(2)], \sigma(3)], \ldots], \sigma(n)] \text {, }
$$

where $\sigma$ describes all permutations over $\{2,3, \ldots, n\}$, is a basis of $L_{1^{n}}(\{1,2, \ldots, n\})$. In particular, every element $P$ of $L_{1^{n}}(\{1,2, \ldots, n\})$ may be decomposed in this basis and therefore encoded by an element of the $\mathbb{Z}$-algebra of the symmetric group $\mathfrak{S}_{\{2, \ldots, n\}}$. Since this last algebra is canonically isomorphic to the $\mathbb{Z}$-algebra of $\mathfrak{S}_{n-1}$, we can define a natural mapping $\tau$ from $L_{1^{n}}(\{1,2, \ldots, n\})$ into $\mathbb{Z}\left[\mathfrak{S}_{n-1}\right]$ as follows : for every Lie polynomial $P$ of $L_{1^{n}}(\{1,2, \ldots, n\})$ uniquely decomposed as

$$
P=\sum_{\sigma \in \mathfrak{S}_{\{2, \ldots, n\}}} P_{\sigma}[\sigma]
$$

one sets

$$
\tau(P)=\sum_{\sigma \in \mathfrak{S}_{n-1}} P_{\sigma^{\prime}} \sigma
$$

where $\sigma^{\prime}$ is the permutation of $\mathfrak{S}_{\{2, \ldots, n\}}$ defined by

$$
\sigma^{\prime}(i)=\sigma(i)+1
$$

for $i \in[1, n-1]$. We can now describe $l_{n}(\pi)$ for every Lie element $\pi$ of $\mathbf{S y m}_{n}$.

Theorem 9.2 Let $\pi$ be a Lie quasi-idempotent of $\mathbf{S y m}_{n}$. Then, the element $\tau\left(l_{n}(\pi)\right)$ belongs to $\Sigma_{n-1}$. One can associate with it the element $\tau_{n}(\pi)$ of $\mathbf{S y m}_{n-1}$ defined by

$$
\tau_{n}(\pi)=\alpha\left(\tau\left(l_{n}(\pi)\right)\right)
$$

Then, in Sym

$$
\tau_{n}(\pi)=\Lambda_{1}^{-1} \cdot \pi .
$$


Proof - Let us first introduce some notations. For $x \in \mathbb{Z}\left[\mathfrak{S}_{n-1}\right]$, we denote by $i_{n-1}^{n}(x)$ the element of $\mathbb{Z}\left[\mathfrak{S}_{n}\right]$ corresponding to $x$ in the identification

$$
\mathfrak{S}_{n-1}=\operatorname{Fix}(1) \subset \mathfrak{S}_{n},
$$

where $F i x(1)$ is the set of permutations fixing 1 . For $x \in \mathbb{Z}\left[\mathfrak{S}_{n}\right]$, we denote by $x \cap F i x(1)$ the element defined by

$$
x \cap F i x(1)=\sum_{\sigma \in F i x(1)}(x \mid \sigma) \sigma .
$$

Let now $\pi$ be a Lie quasi-idempotent of $\mathbf{S y m}_{n}$ and let

$$
\pi=\sum_{i \geq 2} a_{i I} R_{i I}+\sum_{I} a_{1 I} R_{1 I}
$$

be its decomposition on the ribbon basis. According to Proposition 9.1,

$$
\Lambda_{1}^{-1} \cdot \pi=\sum_{i \geq 2} a_{i I} R_{i-1, I}
$$

Let us now identify the elements of Sym with their inverse images under $\alpha$ in $\mathbb{Z}\left[\mathfrak{s}_{n}\right]$. It is then easy to see that

$$
i_{n-1}^{n}\left(\Lambda_{1}^{-1} \cdot \pi\right)=\left(\sum_{i \geq 2} a_{i I} R_{i I}\right) \cap F i x(1) .
$$

Since one always has $R_{1 I} \cap F i x(1)=0$, it follows from this last equality that

$$
i_{n-1}^{n}\left(\Lambda_{1}^{-1} \cdot \pi\right)=\pi \cap \operatorname{Fix}(1) .
$$

The theorem will follow from this last formula, and from the following lemma.

Lemma 9.3 Let $\pi$ be a Lie element in $\mathbb{Z}\left[\mathfrak{s}_{n}\right]$. Then,

$$
\pi=(\pi \cap \operatorname{Fix}(1)) \theta_{n},
$$

where $\theta_{n}$ denotes Dynkin's idempotent.

Proof - It suffices to prove the lemma when $\pi$ is a Lie element of the form $\pi=\left[P_{1}, Q\right]$ where $P_{1}$ and $Q$ are alternants of lower degrees, $P_{1}$ being the alternant containing the letter 1 . The proof goes by induction on the degree $d$ of $P_{1}$. If $d=1$, we have

$$
\pi=[1, Q]=1 Q-Q 1
$$

In this case, the lemma reduces to

$$
\pi=(1 Q) \theta_{n},
$$

which follows from Dynkin's criterion and from an identity of Baker (see formula (1.6.5) p. 36 of [33]). Suppose now that $P_{1}$ is of degree $d>1$. Then we have

$$
\pi \cap F i x(1)=\left(P_{1} Q-Q P_{1}\right) \cap F i x(1)=\left(P_{1} \cap F i x(1)\right) Q .
$$

Using again Baker's formula, Dynkin's criterion and the induction hypothesis, we obtain

$$
(\pi \cap F i x(1)) \theta_{n}=\left(\left(P_{1} \cap F i x(1)\right) Q\right) \theta_{n}=\left[\left(P_{1} \cap F i x(1)\right) \theta_{d}, Q\right]=\left[P_{1}, Q\right] .
$$


Note 9.4 Using the notations of the proof of Proposition 9.2 and identifying again noncommutative symmetric functions with their images by $\alpha$, Proposition 9.2 can be equivalently restated

$$
\pi=\theta_{n} i_{n-1}^{n}\left(\Lambda_{1}^{-1} \cdot \pi\right)
$$

for any Lie quasi-idempotent $\pi$ in $\Sigma_{n}$.

Example 9.5 Let us consider the Lie quasi-idempotent $\pi=\left[\Psi_{1}, \Psi_{2}\right]$. Its inverse image under $\alpha$ is

$$
\pi^{\prime}=\alpha(\pi)=2(213+312-132-231)=-2[[1,3], 2]=-2[(32)],
$$

so that $\tau\left(l_{2}(\pi)\right)=-2(21)$. From this, one gets

$$
\tau_{2}(\pi)=-2 \Lambda_{2}
$$

in agreement with formula (180). Indeed,

$$
\pi=\Psi^{12}-\Psi^{21}=-2 \Lambda^{12}+2 \Lambda^{21}
$$

whence $\Lambda_{1}^{-1} \cdot \pi=-2 \Lambda_{2}$.

As an immediate consequence of the last proposition, we can state :

Corollary 9.6 The restriction of the left derivative $\Lambda_{1}^{-1}$ to $L(\Psi)$ is injective.

Note 9.7 Corollary 9.6 can also be directly obtained as follows. It suffices to show that $l=0$ is the only homogeneous Lie element of $L(\Psi)$ such that $\Lambda_{1}^{-1}(l)=0$. Let then $l$ be a non-zero Lie element of $L(\Psi)$ such that $\Lambda_{1}^{-1}(l)=0$. This means that

$$
l=\sum_{i+|I|=n, i \geq 2} c_{i, I} \Lambda_{i} \Lambda^{I}
$$

Let $I_{0}$ be the maximal (for the lexicographic order) composition appearing in (182) and let $i_{0}=n-\left|I_{0}\right|$. It is then easy to see that the element $\Lambda_{1} \otimes \Lambda_{i_{0}-1} \Lambda^{I_{0}}$ will appear with a non-zero coefficient in the expansion of $\Delta(l)$. But this is not possible since $l$ is primitive for $\Delta$. This contradiction shows that $l$ must be equal to 0 .

\subsection{Decompositions on other Lie bases}

We showed in the previous subsection how to decompose a Lie quasi-idempotent $\pi$ of $\Sigma_{n}$ on Dynkin's basis $\left(\sigma \theta_{n}\right)_{\sigma \in \text { Fix (1) }}$. It appeared that one has

$$
\pi=i_{n-1}^{n}\left(\Lambda_{1}^{-1} \pi\right) \theta_{n} .
$$

In other words, the encoding used for the decomposition of $\pi \in \Sigma_{n}$ on Dynkin's basis lies in the descent algebra $\Sigma_{n-1}$ and can be described in terms of noncommutative symmetric functions of $\mathbf{S y m}_{n-1}$. This is in fact a general property.

Proposition 9.8 Let $f_{n}$ be a Lie idempotent in $\Sigma_{n}$ such that the family $\left(\sigma f_{n}\right)_{\sigma \in F i x(1)}$ is a basis of $L_{1^{n}}(1,2, \ldots, n)$. Let $\pi$ be an arbitrary Lie quasi-idempotent of $\Sigma_{n}$. Then, there exists an element $s\left(\pi, f_{n}\right)$ of $\Sigma_{n-1}$ such that

$$
\pi=i_{n-1}^{n}\left(s\left(\pi, f_{n}\right)\right) f_{n} .
$$


Proof - The decomposition of $f_{n}$ on Dynkin's basis is given by

$$
f_{n}=i_{n-1}^{n}\left(\Lambda_{1}^{-1} f_{n}\right) \theta_{n} .
$$

On the other hand,

$$
\theta_{n}=i_{n-1}^{n}\left(T\left(f_{n}\right)\right) f_{n}
$$

where $T\left(f_{n}\right)$ is some element of $K\left[\mathfrak{S}_{n-1}\right]$. The last two formulas imply that $\Lambda_{1}^{-1} f_{n}$ is necessarily invertible (for the internal product) and that $T\left(f_{n}\right)$ is its inverse. Hence $T\left(f_{n}\right)$ belongs to $\Sigma_{n-1}$. It follows that

$$
\pi=i_{n-1}^{n}\left(\Lambda_{1}^{-1} \pi\right) \theta_{n}=i_{n-1}^{n}\left(\left(\Lambda_{1}^{-1} \pi\right) * T\left(f_{n}\right)\right) f_{n}
$$

Note 9.9 The above proof reduces the computation of $s\left(\pi, f_{n}\right)$ to the inversion of $\Lambda_{1}^{-1} f_{n}$ for the internal product.

Klyachko's idempotent $\kappa_{n}(\zeta)$ of order $n$ is a Lie idempotent of $\Sigma_{n}$ for which the family $\left(\sigma \kappa_{n}(\zeta)\right)_{\sigma \in \text { Fix (1) }}$ is a basis of $L_{1^{n}}(1,2, \ldots, n)$ that we will call Klyachko's basis (see [33]). One can therefore use the above techniques to compute the decomposition of an arbitrary Lie quasi-idempotent of $\Sigma_{n}$ on this basis. To this purpose, we essentially need to know the decomposition of Dynkin's idempotent on Klyachko's basis which is given by the following proposition.

Proposition 9.10 Let be a primitive $n$-th root of unity. Then,

$$
\theta_{n}=i_{n-1}^{n}\left(\left.S_{n-1}((1-q) A)^{*}\right|_{q=\zeta^{-1}}\right) \kappa_{n}(\zeta)
$$

Proof - Going back to the definition of $\kappa_{n}(\zeta)$, one can write

$$
\Lambda_{1}^{-1} \kappa_{n}(\zeta)=\frac{1}{n} \sum_{|I|=n, i_{1} \geq 2} \zeta^{\operatorname{maj}(I)} R_{i_{1}-1, i_{2}, \ldots, i_{r}}
$$

the above sum being taken over all compositions $I=\left(i_{1}, \ldots, i_{r}\right)$ of $n$ with first part greater or equal to 2 . Since we clearly have maj $\left(i_{1}-1, i_{2}, \ldots, i_{r}\right)=$ maj $\left(i_{1}, \ldots, i_{r}\right)+r-1$ when $i_{1} \geq 2$, we get

$$
\Lambda_{1}^{-1} \kappa_{n}(\zeta)=\frac{1}{n} \sum_{|I|=n-1} \zeta^{\operatorname{maj}(I)+l(I)-1} R_{I}
$$

Observe now that one always has

$$
\operatorname{maj}(I)+\operatorname{maj}(\bar{I})=(n-1)(\ell(I)-1) \equiv 1-\ell(I)[n]
$$

for every composition $I$ of $n-1$. It follows that one can rewrite the last identity as

$$
\Lambda_{1}^{-1} \kappa_{n}(\zeta)=\frac{1}{n} \sum_{|I|=n-1} \zeta^{-\operatorname{maj}(\bar{I})} R_{I}=\left.\left(\frac{1}{(q)_{n-1}} \sum_{|I|=n-1} q^{\operatorname{maj}(I)} R_{\bar{I}}\right)\right|_{q=\zeta^{-1}}
$$


This means that

$$
\Lambda_{1}^{-1} \kappa_{n}(\zeta)=\left.S_{n-1}\left(\frac{A}{1-q}\right)^{*}\right|_{q=\zeta^{-1}}
$$

Hence,

$$
\kappa_{n}(\zeta)=i_{n-1}^{n}\left(\left.S_{n-1}\left(\frac{A}{1-q}\right)^{*}\right|_{q=\zeta^{-1}}\right) \theta_{n},
$$

and inverting this last equality gives the desired relation.

Using the last two propositions, one can now give the explicit decomposition of any Lie quasi-idempotent $\pi$ of $\Sigma_{n}$ on Klyachko's basis:

$$
\pi=i_{n-1}^{n}\left(\left.\left(\Lambda_{1}^{-1} \pi\right) * S_{n-1}((1-q) A)^{*}\right|_{q=\zeta^{-1}}\right) \kappa_{n}(\zeta) .
$$


[1] M.D. Atkinson, Solomon's descent algebra revisited, Bull. London Math. Soc. 24 (1992), 545-551.

[2] F. Bergeron, N. Bergeron and A.M. Garsia, Idempotents for the free Lie algebra and qenumeration, in Invariant theory and tableaux, D. Stanton ed., IMA Volumes in Mathematics and its Applications, Vol. 19, Springer, 1988.

[3] F. Bergeron, A. Garsia and C. Reutenauer, Homomorphisms between Solomon's descent algebras, J. Algebra 150 (1992), 503-519.

[4] I. Bialynicki-Birula, B. Mielnik and J. Plebański, Explicit solution of the continuous Baker-Campbell-Hausdorff problem, Annals of Physics 51 (1969), 187-200.

[5] D. Blessenohl and H. Laue, Algebraic combinatorics related to the free Lie algebra, Actes du 29-ième Séminaire Lotharingien de Combinatoire, A. Kerber Ed., Publ. IRMA, Strasbourg, 1993, 1-21.

[6] A.R. Calderbank, P. Hanlon and S. Sundaram, Representations of the symmetric group in deformations of the free Lie algebra, Trans. Amer. Math. Soc 341 (1994), 315-333.

[7] G. Duchamp, Orthogonal projection onto the free Lie algebra, Theor. Comp. Sci. 79 (1991), 227-239.

[8] G. Duchamp, D. Krob, B. Leclerc and J.-Y. Thibon, Déformations de projecteurs de Lie, C.R. Acad. Sci. Paris 319 (1994), 909-914.

[9] E.B. Dynkin, Calculation of the coefficients in the Campbell-Baker-Hausdorff formula, Dokl. Akad. Nauk. SSSR (N.S.) 57 (1947), 323-326 (in Russian).

[10] A.M. Garsia, Combinatorics of the free Lie algebra and the symmetric group, in Analysis, et cetera ..., Jürgen Moser Festschrift, Academic press, New York, 1990, 309-82.

[11] A.M. Garsia and C. Reutenauer, A decomposition of Solomon's descent algebra, Advances in Math. 77 (1989), 189-262.

[12] I.M. Gelfand, D. Krob, A. Lascoux, B. Leclerc, V.S. Retakh and J.-Y. Thibon, Noncommutative symmetric functions, Adv. in Math. 112 (1995), 218-348.

[13] I.M. Gelfand and V.S. Retakh, Determinants of matrices over noncommutative rings, Funct. Anal. Appl. 25 (1991), 91-102.

[14] I.M. Gelfand and V.S. Retakh, A theory of noncommutative determinants and characteristic functions of graphs, Funct. Anal. Appl. 26 (1992), 1-20; Publ. LACIM, UQAM, Montreal, 14, 1-26.

[15] I. Gessel, Multipartie P-partitions and inner product of skew Schur functions, Contemp. Math. 34 (1984), 289-301.

[16] I. Gessel and C. Reutenauer, Counting permutations with given cycle structure and descent set, J. Comb. Theory A 64 (1993), 189-215.

[17] A.A. Klyachko, Lie elements in the tensor algebra, Siberian Math. J. 15 (1974), 1296-1304.

[18] D. Krob and B. LeClerc, Minor identities for quasi-determinants and quantum determinants, Commun. Math. Phys. 169 (1995), 1-23.

[19] A. Lascoux and M.P. Schützenberger, Formulaire raisonné de fonctions symétriques, Publ. Math. Univ. Paris 7, Paris, 1985.

[20] J. L. Loday, Opérations sur l'homologie cyclique des algèbres commutatives, Invent. Math., 96, (1989), 205-230.

[21] I.G. Macdonald, Symmetric functions and Hall polynomials, Oxford Math. Monographs, Oxford University Press, 2nd ed., 1994. 
[22] P.A. MacMahon, Combinatory analysis, Cambridge University Press, 1915, 1916; Chelsea reprint, 1960.

[23] W. Magnus, On the exponential solution of differential equations for a linear operator, Comm. Pure Appl. Math. VII (1954), 649-673.

[24] C. Malvenuto and C. Reutenauer, Duality between quasi-symmetric functions and the Solomon descent algebra, J. Algebra 177 (1995), 967-982.

[25] B. Mielnik and J. Plebański, Combinatorial approach to Baker-Campbell-Hausdorff exponents, Ann. Inst. Henri Poincaré, Section A, vol. XII, (1970), 215-254.

[26] A.I. Molev, Noncommutative symmetric functions and Laplace operators for classical Lie algebras, Preprint, 1994.

[27] P. Moszkowski, Généralisation d'une formule de Solomon relative à l'anneau d'un groupe de Coxeter, C.R. Acad. Sci. Paris 309 (1989), 539-541.

[28] F. Patras, Construction géométrique des idempotents eulériens. Filtration des groupes de polytopes et des groupes d'homologie de Hochschild, Bull. Soc. Math. France 119 (1991), 173198.

[29] F. Patras, L'algèbre des descentes d'une bigèbre graduée, J. Algebra 170 (1994), 547-566.

[30] F. PATras, Opérations d'Adams et gamma-filtration non-commutatives, preprint Univ. Nice, 1994.

[31] A. RAm, A Frobenius formula for the characters of Hecke algebras, Invent. Math. 106 (1991), 461-488.

[32] C. Reutenauer, Theorem of Poincaré-Birkhoff-Witt, logarithm and representations of the symmetric group whose order are the Stirling numbers, in Combinatoire énumérative, Proceedings, Montréal 1985 (G. Labelle and P. Leroux Eds.), Lecture Notes in Math. 1234, Springer, (1986), 267-284.

[33] C. Reutenauer, Free Lie algebras, Oxford University Press, 1993.

[34] L. Solomon, A Mackey formula in the group ring of a Coxeter group, J. Algebra, 41, (1976), 255-268.

[35] L. Solomon, On the Poincaré-Birkhoff-Witt theorem, J. Comb. Th. 4 (1968), 363-375.

[36] W. Specht, Die linearen Beziehungen zwischen höheren Kommutatoren, Math. Zeit. 51 (1948), 367-376.

[37] B.C.V. UNG, NCSF, a MAPLE package for noncommutative symmetric functions, Maple Technical Newsletter (1996), to appear.

[38] F. Wever, Über Invarianten in Lieschen Ringen, Math. Annalen 120 (1949), 563-580.

[39] R.M. Wilcox, Exponential operators and parameter differentiation in Quantum Physics, J. Math. Phys. 8 (1967), 962-982. 